LOCT 252011

\title{
Integration of Radio over Fiber (RoF) with Fiber To The Home (FTTH) Schemes
}

\author{
FROPERTY OF
TIERSON UNIVESITY LIERARV \\ by \\ Yonas Endailalu
}

Project Report

Presented to Ryerson University and Karlsruhe University of Applied Sciences

in partial fulfillment of the requirements for the double degrees of Master of Engineering

In Departments of

Electrical and Computer Engineering

Ryerson University and Karlsruhe University of Applied Sciences

Toronto, Canada

Karlsruhe, Germany

(C) Yonas Endailalu,2011 


\section{Author's Declaration}

I hereby declare that I am the sole author of this project report.

I authorize Ryerson University and Karlsruhe University of Applied Sciences to lend this project report to other institutions or individuals for the purpose of scholarly research.

Author's signature : .,

I further authorize Ryerson University and Karlsruhe University of Applied Sciences to reproduce this project report by photocopying or by other means, in total or in part, at the request of other institutions or individuals for the purpose of scholarly research.

Author's signature : 


\section{Access to Project}

Ryerson University and Karlsruhe University of Applied Sciences require the signature of all persons using or photocopying this project report. Please sign below and give address and date.

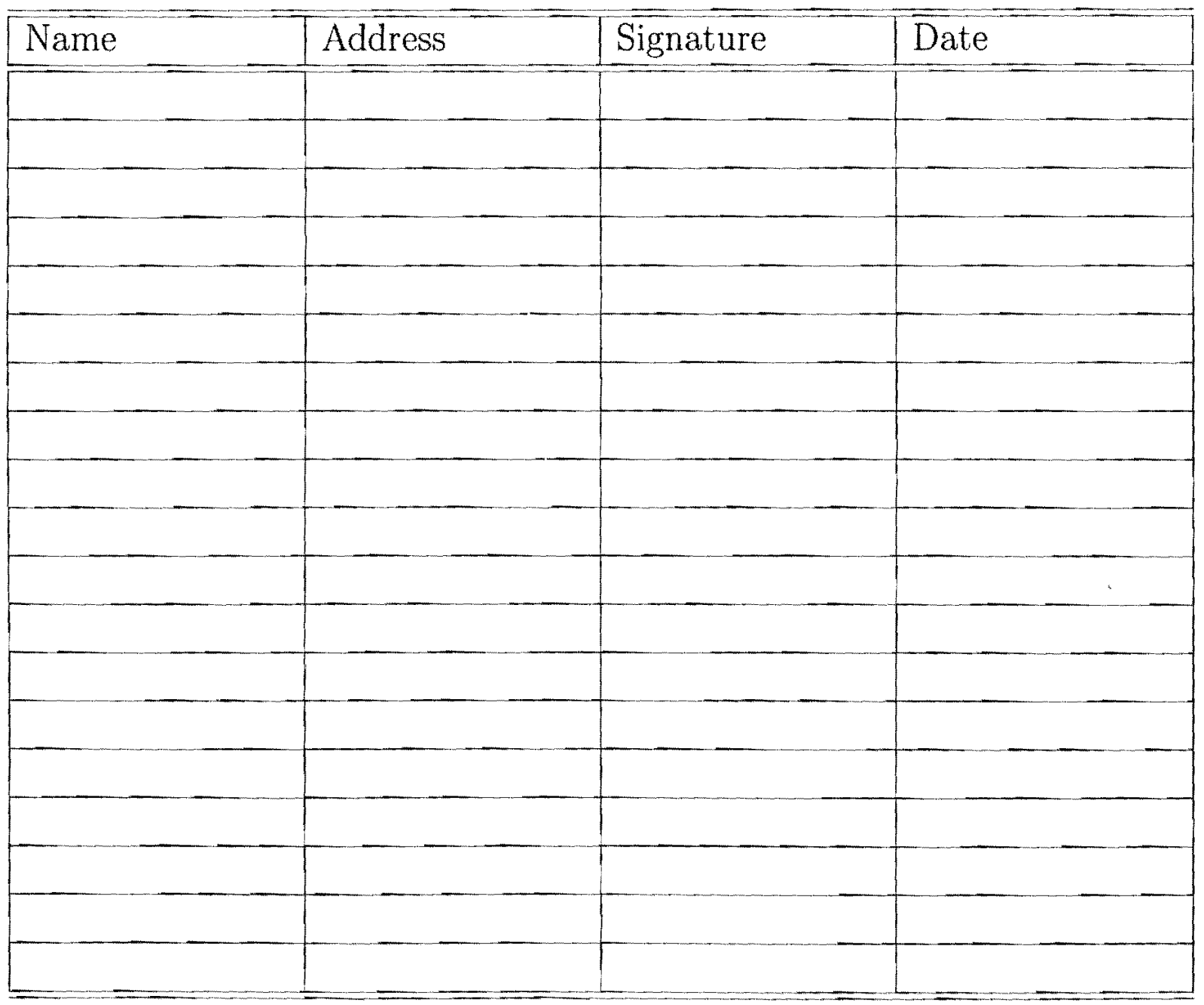




\section{Acknowledgment}

I take this opportunity to convey my warmest thanks to my parents and brothers whose endless support and encouragement brought me all the way through.

I would like to express my deep sense of gratitude to my supervisors, Prof. Xavier Fernando of Ryerson University and Prof. Franz Quint of Karlsruhe University of Applied Sciences for their invaluable help and guidance during the course of the project. I am highly indebted to them for the constant encouragement, feedback and suggestions. I am grateful to them for having given me the support and confidence.

I would also like to thank my friends, Kebe and Semir, electrical engineering graduates of Ryerson University, for their invaluable editorial help and comments on the preparation of the report and presentation materials.

Finally, I would like to thank all my family and friends who directly or indirectly involved in the completion of the project. 


\begin{abstract}
Two integrating techniques and topologies for fiber optic network systems such as RoF and FTTH have been studied and presented in this project. Two digital signals with data rate $622 \mathrm{Mb} / \mathrm{s}$ and $1.25 \mathrm{~Gb} / \mathrm{s}$ are considered as the two service signals to be hybrid. Both signals are first modulated in electrical domain by $25 \mathrm{GHz}$ and $5 \mathrm{GHz}$ sinusoidal subcarriers respectively, in order to give them enough spectral separation. Two architectures have been studied to modulate the optical carrier with these two service signals. The first model is to sum the two service signals in the electric domain and use a single leg Mach Zender Modulator (MZM) to modulate the optical carrier with the electrical message signal. In the second case, the two service signals are first sent to a two leg MZM and separately modulate the light wave carrier, then the two emerging light wave signals mix at the other end of the $Y$ branch of the MZM. The mathematical equations that represent the function of the two kinds of MZM have been formulated. Simulations of the integration of the two hybrid services have been made in MATLAB-Simulink environment and the results are shown. The developed mathematical models are used to represent the MZMs in the MATLAB program. Detector models for each architecture have been developed in order to evaluated the SNR and compare performance using Bit Error Rate (BER). The BER plots for different input setup have been plotted and presented in this project.
\end{abstract}




\section{Contents}

1 Introduction 1

1.1 Overview of Fiber-Optics . . . . . . . . . . . . . . . . . 1

1.2 Fiber To The Home $(\mathrm{FTTH}) \ldots \ldots \ldots \ldots$

1.3 Radio over Fiber $(\mathrm{RoF}) \ldots \ldots \ldots \ldots$

1.4 Contribution . . . . . . . . . . . . . . . 5

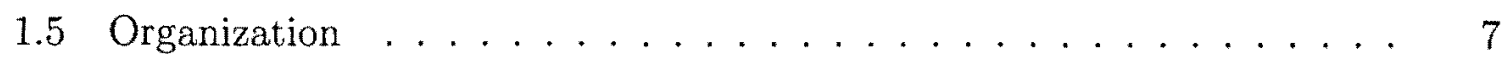

2 Fiber To The Home $\quad 9$

2.1 FTTH Development . . . . . . . . . . . . . . . . 9

2.2 PON Systems . . . . . . . . . . . . . . . . . . . . . . 11

2.3 PON Standardization . . . . . . . . . . . . . . . . . 14

2.4 EPON/GEPON . . . . . . . . . . . . . . . . . . 15

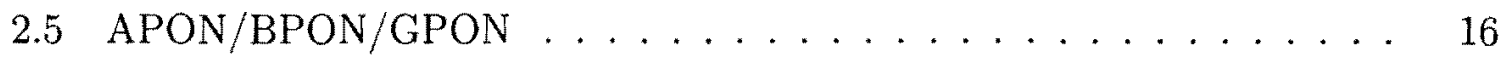

3 Radio over Fiber $\quad 18$

3.1 RoF Basics . . . . . . . . . . . . . . . . . . . . 18

3.2 Advantage of RoF . . . . . . . . . . . . . . 20

3.2 .1 Low Complexity. . . . . . . . . . . . . . . . . . . 20

3.2 .2 Low Attenuation . . . . . . . . . . . . . . . 21

3.2 .3 Low Cost . . . . . . . . . . . . . . . . . . 21

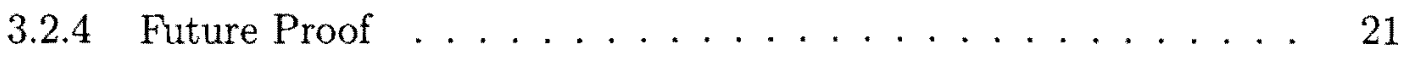

3.3 Application of RoF . . . . . . . . . . . . . 22

4 Hybrid Fiber Optic Services $\quad 23$ 
5 Mathematical Models $\quad 30$

5.1 Modulation Model . . . . . . . . . . . . . . . . . . . . . 30

5.1 .1 Pockel's Effect . . . . . . . . . . . . . . . . . 31

5.1.2 Y-branch Interfrometeric Mach Zender Modulator (MZM) . . . 33

5.1 .3 Single Signal Model Equation . . . . . . . . . . . . . . 34

5.1 .4 Dual Signal Model Equation . . . . . . . . . . . . . . . . . . . 37

5.2 Channel Model . . . . . . . . . . . . . . . . . . . . . 41

5.3 Detection Model . . . . . . . . . . . . . . . . . . 42

5.3 .1 Photo Detector Noise . . . . . . . . . . . . . . . . . . . 44

5.4 BER Determination . . . . . . . . . . . . . . . . 46

5.4.1 BER for One Leg Input Signal Model . . . . . . . . . . . . 49

5.4 .2 BER for Two Leg Input Signal Model . . . . . . . . . . . . 50

6 Simulation and Results $\quad 51$

6.1 Simulation Setup . . . . . . . . . . . . . . . . . 51

6.2 One Leg Model . . . . . . . . . . . . . . . . . . . . . . 53

6.3 Two Leg Model . . . . . . . . . . . . . . . . . . . . . . 60

6.4 BER Plots . . . . . . . . . . . . . . . . . 66

7 Conclusion and Remarks $\quad \mathbf{7 2}$

7.1 Conclusion . . . . . . . . . . . . . . . . 72

7.2 Future Work . . . . . . . . . . . . . . . . . 74

$\begin{array}{lr}\text { References } & \mathbf{7 5}\end{array}$

Yonas Endailalu, B.Eng. vii 


\section{List of Figures}

1.1 Historical development of the three generations of fiber optics. . . . . . 2

1.2 Example of Fiber-To-The-Home system. . . . . . . . . . . . . . 4

1.3 Basic layout of Radio over Fiber $(\mathrm{RoF})$ system $\ldots \ldots \ldots$

2.1 Different layers for the fiber optic network system. . . . . . . . . 10

2.2 A pictorial representation of the major services provided by FTTH. . . 11

2.3 Comparison of subscriber growth rate in competing technologies . . . . 12

2.4 PON distribution architecture topology . . . . . . . . . . . . . 13

2.5 Three major kinds of PON topologies. . . . . . . . . . . . 14

2.6 Pictorial representation of PON standard's timeline and their deployment. 15

3.1 Representation of RoF for millimeter wave wireless access system. . . . 19

4.1 The schematic diagram for the hybrid RoF services using single leg MZM. 26

4.2 The schematic diagram for the hybrid RoF services using two leg MZM. 27

5.1 A structural representation of the one signal input $\mathrm{Y}$ branch MZM . . 34

5.2 A physical structure representation of the two signal input $\mathrm{Y}$ branch MZM. 38

6.1 Block diagram of the signal flow for the simulation models. . . . . . 52

6.2 Block diagram of the flow of signal in Simulink phase of the simulation. 54

6.3 Block diagram for the signal flow in the MATLAB modules. . . . . . 54

6.4 Block diagram for the Simulink stage following the MATLAB processes. 55

6.5 The schematic diagram for simulation of one leg input model . . . . 56

6.6 Time domain simulation display of the two input signals in Simulink

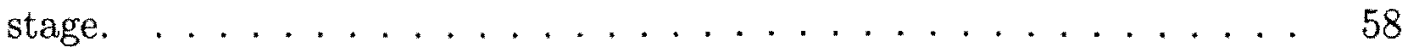


6.7 Time domain simulation display comparison at Simulink and MATLAB

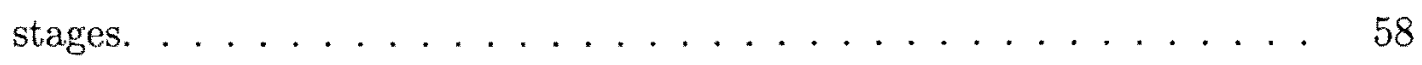

6.8 Frequency spectrum display for the simulation of single signal input model. . . . . . . . . . . . . . . . . 59

6.9 Block diagram for the simulation source for radio signal 1 in Simulink. 61

6.10 Block diagram for the simulation source for radio signal 2 in Simulink. 61

6.11 The schematic diagram representation of the two radio signal input model. 62

6.12 Time domain simulation display of the two input signals in Simulink stages. 64

6.13 Time domain simulation display of the two input signals after optical carrier modulation in MATLAB. . . . . . . . . . . . . 64

6.14 Frequency spectrum display for the simulation of two signal input model. 65

6.15 BER plot comparison for two models with $\alpha$ and $\beta$ varying simultaneously 67

6.16 BER plot comparison for two models $\alpha$ fixed and $\beta$ varying . . . . . . 68

6.17 BER plot comparison for two models with $\alpha$ and $\beta$ varying, thermal noise included . . . . . . . . . . . . . . . . 69

6.18 BER plot with both $\alpha$ and $\beta$ fixed, optical power varying, no thermal noise 70

6.19 BER plot with both $\alpha$ and $\beta$ fixed, varying optical power, thermal noise included ........................ 71 


\section{List of Acronyms and Abbreviations}

$\begin{array}{ll}\text { ADPCM } & \text { Adaptive Differential Pulse Coded Modulation } \\ \text { ADSL } & \text { Asymmetrical Digital Subscriber Line } \\ \text { AMPS } & \text { Advanced Mobile Phone System (TIA 553) } \\ \text { ANSA } & \text { Advanced Network System Architecture } \\ \text { ANSI } & \text { American National Standards Institute (NY) } \\ \text { AON } & \text { Active Optical Network } \\ \text { APD } & \text { Avalanche Photo Diode } \\ \text { APPN } & \text { Advanced Peer to Peer Networking (IBM) } \\ \text { ASK } & \text { Amplitude Shift Keying } \\ \text { AT \& T } & \text { American Telegraph \& Telephone } \\ \text { ATM } & \text { Asynchronous Transfer Mode (Cell-Switching, Fast Packet) } \\ \text { BER } & \text { Bit Error Rate } \\ \text { BERT } & \text { bit Error Rate Tester } \\ \text { B-ICI } & \text { Broad band Inter-Carrier Interface } \\ \text { bit } & \text { BInary digiT } \\ \text { BRON } & \text { Broadband Passive Optical Network } \\ \text { BPSK } & \text { Binary Phase Shift Keying } \\ \text { BSS } & \text { Broad band Switching System } \\ \text { CATV } & \text { Community Antenna (CAble) TeleVision } \\ \text { CDMA } & \text { Code Division Multiple Access (Cellular, IS-95) } \\ \text { CSA } & \text { Canadian Standards Association (Rexdale) } \\ \text { CSMA/CD } & \text { Carrier Sense with Multiple Access/Collision Detection (Ethernet } \\ \text { CW } & \text { IEEE 802.3) } \\ \text { DCS } & \text { Continuous Wave } \\ \text { DFSK } & \text { Dynamic Channel Selection } \\ \text { DOCSIS } & \text { Double Frequency Shift Keying } \\ \text { DPSK } & \text { Data Over Cable Service Interface Specification } \\ \text { DQPSK } & \text { Differential Phase Shift Keying } \\ & \text { Differential Quaternary Phase Shift Keying }\end{array}$


DS/SS

DSL

DSS1

EDFA

EPON

ERP

ETSI

$\mathrm{FC}$

FDDI

FDM

$\mathrm{FH} / \mathrm{SS}$

FITL

FLAG

FOIRL

FOT

FSK

FTTC

FTTH

GaAs

GaAsP

GaN

GMSK

GPON

GSM

HDSL

HDTV

HFC

Hiperlan

IEEE

ILD

IMD

IRED

ISDN

ISO

ITU

ITU-R

ITU-T
Direct Sequence Spread Spectrum

Digital Subscriber Line

Digital Subscriber Signalling System 1 (tel)

Erbium Doped Fiber Amplifier

Ethernet Passive Optical Network

Effective Radiated Power

European Telecommunications Standards Institute (Cedex - F)

Fibre Channel

Fiber Distributed Data Interface (ISO 9314)

Frequency Division Multiplex

Frequency Hopping Spread Spectrum

Fiber In The Loop

Fiber optic Link Around the Globe

Fiber Optic Inter Repeater Link (Ethernet - IEEE 802.3c .3d)

Fiber Optic Transceiver

Frequency Shift Keying

Fiber To The Curb

Fiber To The Home

GAlium ArSenide

GAlium ArSenide Phosphide

GAllium Nitride

Gaussian Minimum Shift Keying

Gegabit Passive Optical Network

Global Standard for Mobile

High rate Digital Subscriber Loop (Line)

High Definition TeleVision

Hybrid Fiber/Coaxial network

HIgh PErformance Radio LAN

Institute of Electric and Electronics Engineers

Injection Laser Diode

InterModulation Distortion

InfraRed Emitting Diode

Integrated Services Data Network

International Standards Organization

International Telecommunications Union

ITU Telecommunications Union - Radio Communications Sector

ITU Telecommunications Union - Telecommunications Sector 


$\begin{array}{ll}\text { LAN } & \text { Local Area Network } \\ \text { LASER } & \text { Light Amplification by Stimulated Emission of Radiation } \\ \text { LCD } & \text { Liquid Crystal Display } \\ \text { LD } & \text { Laser Disc } \\ \text { LED } & \text { Light Emitting Diode } \\ \text { MAN } & \text { Metropolitan Area Networks } \\ \text { MMW } & \text { MilliMeter Waves } \\ \text { MW } & \text { Micro Wave } \\ \text { NA } & \text { Numeric Aperture } \\ \text { NAU } & \text { Network Access Point } \\ \text { OC-1 } & \text { Optical Carrier level 1 (51.84 Mbps) } \\ \text { OCWR } & \text { Optical Continuos Wave Reflectometer } \\ \text { OEIC } & \text { OptoElectronic Integrated Circuit } \\ \text { OLT } & \text { Optical Line Termination } \\ \text { ONT } & \text { Optical Network Terminal } \\ \text { ONU } & \text { Optical Network Unit } \\ \text { OPGW } & \text { overhead OPtical Ground Wave } \\ \text { OTDR } & \text { Optical Time Domain Reflectometer } \\ \text { PCM } & \text { Pulse Code Modulation } \\ \text { PDFA } & \text { Praseodymium Doped Fiber Amplifier } \\ \text { PIN } & \text { Positive Intrinsic Negative (Diode) } \\ \text { PON } & \text { Passive Optical Network } \\ \text { PSK } & \text { Phase Shift Keying } \\ \text { PSTN } & \text { Public Switched Telephone Network } \\ \text { PWM } & \text { Pulsed Wave Modulation } \\ \text { QAM } & \text { Quadrature Amplitude Modulation } \\ \text { RS } & \text { Remote Station } \\ \text { SDMA } & \text { Space Division Multiple Access } \\ \text { SNR } & \text { Signal to Noise Ratio (S/N) } \\ \text { SONET } & \text { Synchronous Optical NETwork } \\ \text { TDMA } & \text { Time Division Multiple Access } \\ \text { VDSL } & \text { Very-high-rate Digital Subscriber Line } \\ \text { WAN } & \text { Wide Area Networks } \\ \text { WDM } & \text { Wave Division Multiplexing } \\ \text { CWDM } & \text { Coarse Wave Division Multiplexing } \\ \text { DWDM } & \text { Dense Wave Division Multiplexing } \\ & \end{array}$




\section{Chapter 1}

\section{Introduction}

\subsection{Overview of Optical Fiber Communications}

The technological advancement in communications has made human life more comfortable. People have been applying the available communication means to facilitate their life and performing different activities they pursue. However, the expansion of current day communication network coverage and the orientation of more and more consumers to different kinds of services impose a series of challenges in providing a reliable and cost effective service. The provision of triple play (voice, data, video) in the communication network demanded a larger information exchange rate in both uplink and downlink directions. The increasingly large demand for high rate information exchange required service providers and researcher to look for advanced technique that can increase the bandwidth capacities of the networks as well as the devices connected. With regard to high band width capacity, fiber optic channels received the highest consideration. Lately, large amount of resource and effort has been directed towards its development and deployment.

Optical communication systems attracted intensive interest since the early research proposed on dielectric surface waveguide for optical frequencies by Kao and Hockham in 1966[19]. Especially in the last three decades optical communication system has been progressed tremendously. There are three distinguished generations for the development of fiber optic communications. Each generation is identified by the window of carrier light wavelength. In the early 1980 's, $800 \mathrm{~nm}$ window wave length has been deployed. 
With multimode optical fiber (MMF), the transmission capacity at this stage was up to $45 \mathrm{Mbits} / \mathrm{sec}$ [19]. Silica-based optical fibers with lower attenuation and window of wavelength $1300 \mathrm{~nm}$ constitute the second generation. Its optical fiber has a capacity of data transfer rate up to $1.7 \mathrm{Gbits} / \mathrm{sec}$. It has improved the chromatic dispersion to be near zero and distance between consecutive repeaters raised to about $50 \mathrm{Km}$ [19]. In the 1990's the third generation optical fiber communication has been evolved with $1550 \mathrm{~nm}$ wavelength window. At the beginning, this system was capable of carrying a data transmission rate of $2.5 \mathrm{Gbits} / \mathrm{sec}$ and also raised the distance between adjacent spacers to $60-70 \mathrm{Km}[19]$. In the early stages the generation of the optical carrier signal is mainly by semiconductor laser sources, and both direct modulation and detection has been taking place.

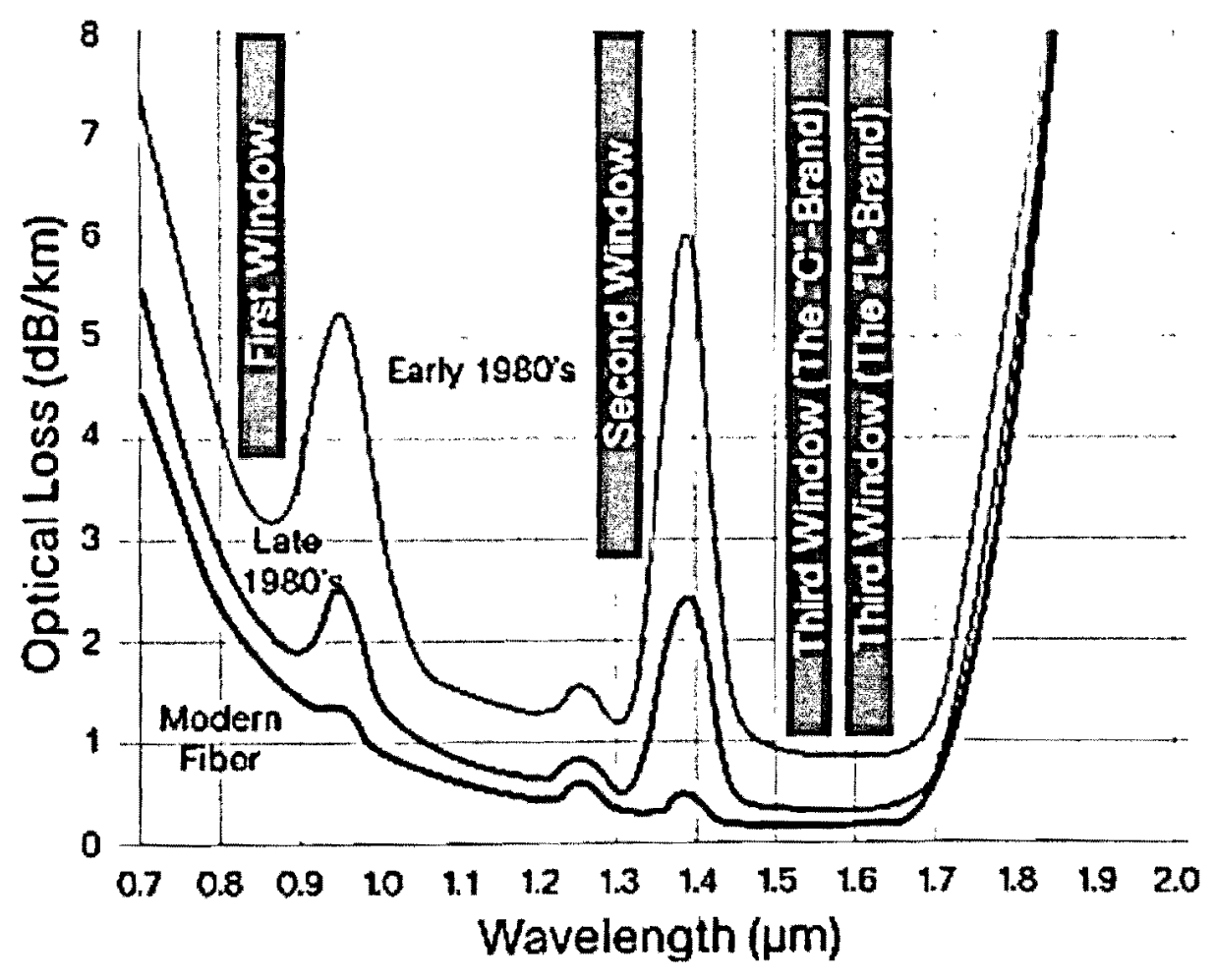

Figure 1.1: Historical development of the three generations and wavelength line windows versus attenuation graph for optical fiber [37].

The invention of EDFA (Erbium-doped Fiber Amplifier) has been huge lap for fiber optic communication. EDFA could be able to provide at least a $20 \mathrm{~dB}$ optical gain. 
Due to such a large gain from EDFA optical loss is no more an issue in the transmission system. WDM (Wavelength Division Multiplexing) and DWDM (Dense-WDM) are subsequently developed technique which enabled multiple of $10 \mathrm{~Gb} / \mathrm{s}$ data signals to be transmitted in a single channel. Current day optical communication systems is sometimes referred to as the fifth generation and have a capacity of data transmission rate up to a few $\mathrm{Tb} / \mathrm{s}$ (Terabit per second)[19].

\subsection{Fiber To The Home (FTTH)}

FTTH is one of the applications of fiber optic communication system. In FTTH the optical fiber line extends from the central station of the service provider to buildings of the work place or at the wall of subscriber home. Once the optical signal arrived at the junction boxes near the home, the message can be further transmitted by several other means such as coaxial cable, twisted wire pair or radio means. FTTH is the extension of the earlier fiber optic system deployments such as Fiber To The Curb (FTTC), Fiber To The Premises (FTTP) and Fiber To The Building (FTTB). Traditionally the baseband signals such as cable TV, home phone and internet service have been provided with copper means such as coaxial cable or twisted wire pair. With extended services and increasingly high demand of the tripe plays these traditional channels could no longer support the data rate requirements of users. In order to make use of large band width capability of optical fiber, basebands services of cable TV, internet and home phone are now provided by fiber optic networks through Fiber- to-the-home (FTTH) system.

\subsection{Radio over Fiber (RoF)}

With the expansion of wireless services, network providers and researchers have shown growing interest in developing broadband systems which is sufficient to deliver data rate for various kinds of services they support. Specially, the provision of wireless internet and wireless communications made the traditional radio system incapable of coping with the ever growing demands of users in the wireless domain. Radio-overFiber (RoF) provided the opportunity of increased bandwidth and extended coverage 


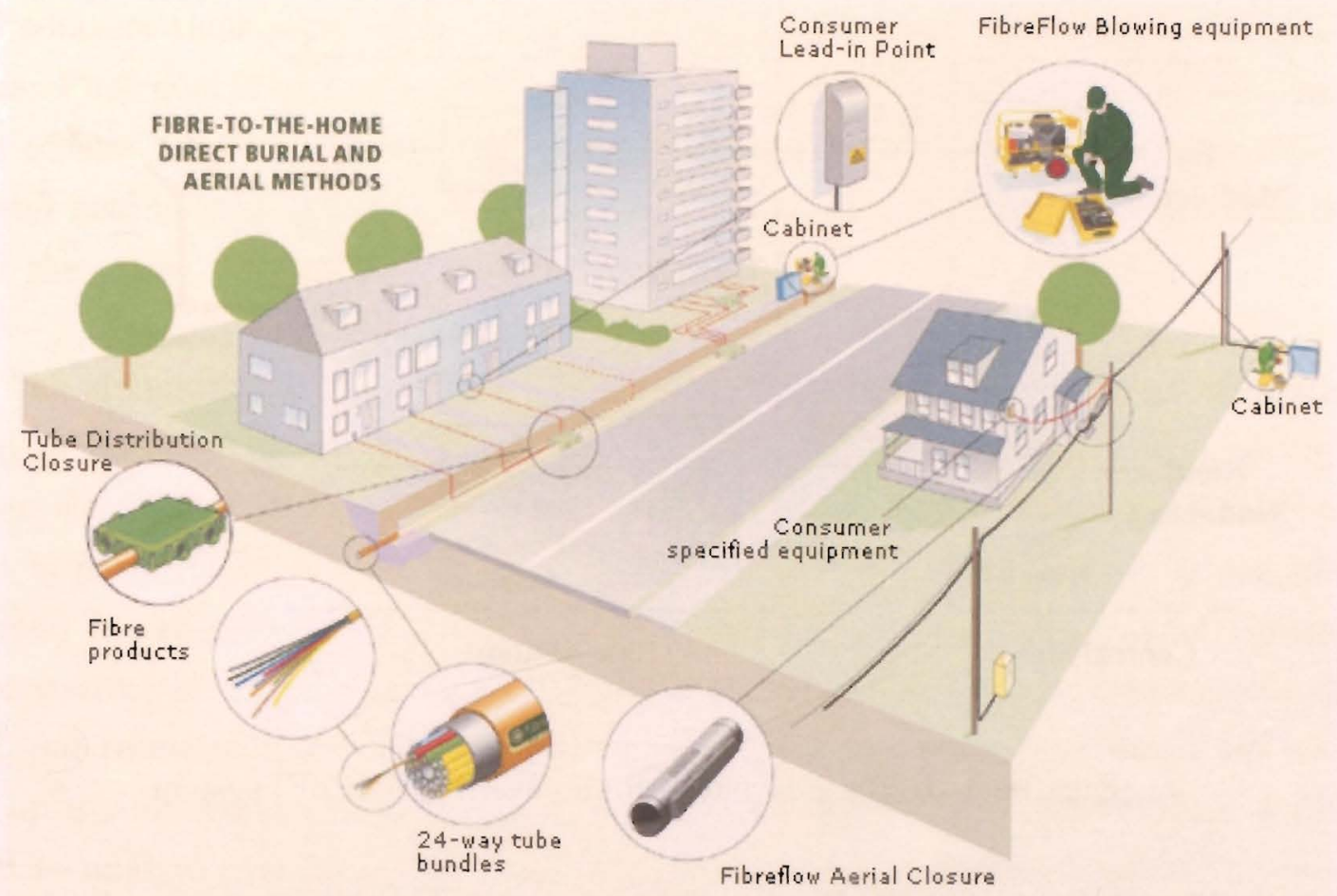

Figure 1.2: Example of Fiber-To-The-Home system [38].

of wireless services over a larger and obstructed area.

RoF become another interesting application technique of fiber optics by which the radio signal is used to modulate light wave to be carried on through optical fiber system. The signal transmission is done through a guided optical fiber instead of open air wireless media. This technique facilitates wireless access and help in realizing seamless wireless communication by providing better distribution of microwave and millimeter wave to far destinations through the optical link. Even though the radio signal could be modulated and carried through optical link for several purposes such as cable television, or satellite base station, we specifically refer RoF when the optical link is used for wireless access.

In order to take the advantage of sharing the optical resources for FTTH and RoF and make use of the full optical spectra obtained by WDM (wavelength division multiplexing), several integrating and interleaving techniques have been developed and 


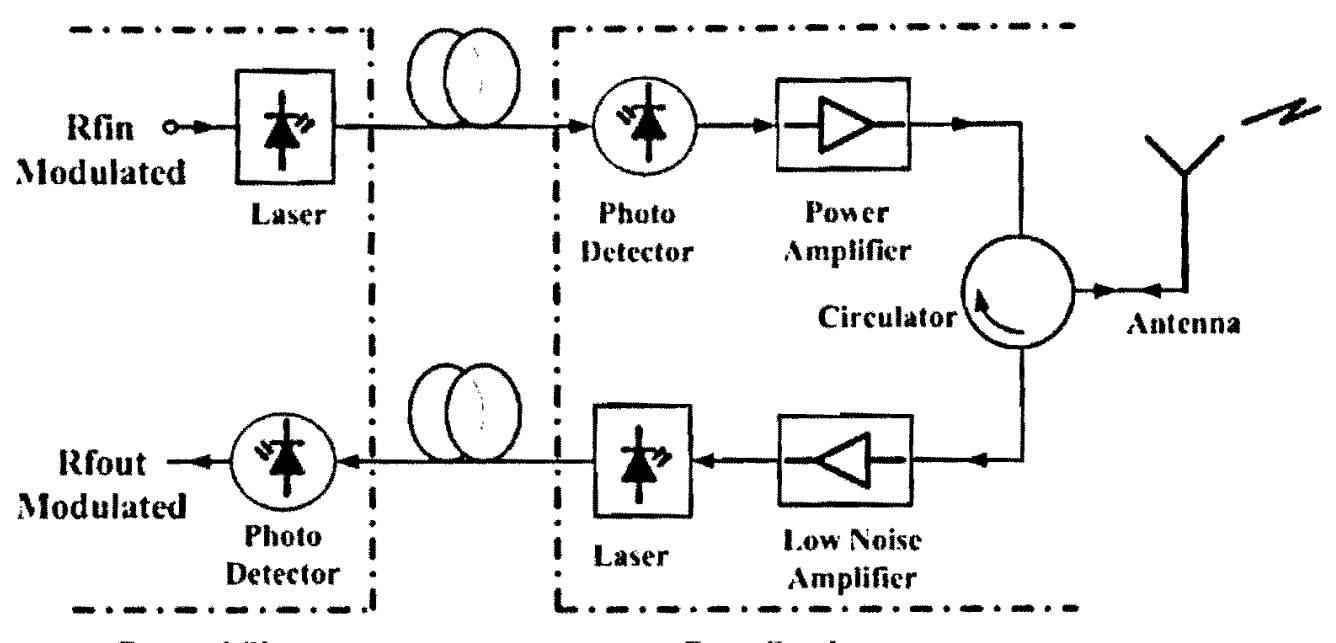

Central Site

Base Station

Figure 1.3: Basic layout of Radio over Fiber (RoF) system

suggested for RoF and FTTH. However, these interleaving techniques require improved modulation, transmission and switching capabilities of the devices and channels involved.

\subsection{Contribution}

Motivated by the integration techniques of RoF and FTTH, this project is conceived and developed to contribute additional information and idea in the integration of two optical fiber based services. In this paper, different technologies and topologies have been investigated for the operating principles and performance of different parts of the network which are used for integrating RoF and FTTH. The project is organized in such a way that it consists of several tasks. The primary task is to setup, develop and perform simulation that can represent the integration of two optical fiber based systems such as RoF and FTTH. Moreover, the project constitutes developing a mathematical model that can represent some important devices and processes which constitute hybrid optical network. Some additional tasks are performed in order to be able to produce alternative modeling architectures for hybrid fiber optic network in order to compare 
and contrast their performance parameters. Generally, the project aimed to meet two goals. First goal is to set up and formulate a mathematical model as well as a simulation scheme that can represent the integration of two optical fiber based services. The second goal is to develop some alternative models and compare the advantages of one over the other.

The simulation is done in MATLAB-Simulink software environment. Simulink is used in its capacity to simulate different signal sources, signal mixing, filtering and other electrical domain based simulation processes. The simulation of the optical part is performed in the MATLAB simulation package. MATLAB programs are developed in order to simulate several optical domain devices such as super continuum light source(the laser), the Mach-Zehnder Modulator (MZM), the transmission channel, and receiver/detector unit. The mathematical equations formulated are used to set up a simulation model for these optical domain devices. The simulink is further used to analyze and investigate the simulation output at several stage of the process. Measurements are done to display both time and frequency domain spectrums. The eye diagrams and performance evaluations are also included in the display. Since the signal changes from electrical domain to light domain and then back to electrical domain, the simulation is done in Simulink - MATLAB - Simulink pattern respective to the signal flow.

The mathematical modeling is developed primarily for the modulation and detection section. Equations are developed based on the basic operating principles of the devices. The optical modulation capability and the opto-electric effect based operation of MZM is the foundation for the mathematical models. With this regard the Pockel's effect, that define the change of refractive index of some material with applied electric field is the basis for the formulation of mathematical equation. Depending on its type and application, MZM might have one or two port for external modulation. In this project, alternative models and architectures are developed based on these number of electrodes. The alternative models are developed based on the application of the modulating electrical signal whether to the one port(one leg model) or to both(two leg model) . 


\subsection{Organization}

In Chapter 2 FTTH technology has been reviewed. Some important definition which will help in understanding FTTH better has been provided. The fast growth rate of FTTH in replacing the existing twisted wire pair and coaxial cable has been shown. Standardization related to FTTH and PON (Passive Optical Network) and its version such as GPON and EPON are also discussed in this chapter. In addition, the past, current and future standard and data bit rate for several PON specification is provided in tabular form. This section is aimed to give the general picture of fast growing application of fiber optic networks and trigger insight on this technology.

Radio over Fiber(RoF) technology has been described in Chapter 3. Again, important definition and terms has been explored that would enhance the understanding of RoF system. The advantage of RoF over other wireless means is described here. An important and descriptive architecture and model is shown there to help clarify the descriptions.

Two alternative hybrid optical network architectures for the integration of two radio service systems have been given in Chapter 4 . The two alternative architectures are conceived and described in order to compare them for performance, ease of deployment and cost issues. The newly developing hybrid fiber optic system is a promising high data rate shared infrastructure integrated system. The architectures have been thought and developed by integrating the two service signals and simultaneously transmitting them at the same optical carrier frequency into the fiber optic networks so that they share the same infrastructure. The alternative architectures provide an insight in developing and investigating several related ideas particularly in the modulation and detection section as well as application of MZM. In 4th Chapter the two architecture are discussed in detail, compared and contrasted.

Chapter 5 is devoted in developing the mathematical equation for the optical part of the model. In this chapter three models are developed which represent the modulation, channel and detection parts of the optical link. Since two alternative modulation models are being considered, two mathematical models and equations have been derived for 
each. The two derived models are identified in their respective subsections as one leg modulation and two leg modulation schemes. In both cases, the modulation operation of MZM is considered and the signal characteristics within the modulator are modeled. The transmission channel is considered for its attenuation and dispersion effects. The equivalent mathematical equation and model for the simulation is provided in this section. At the receiver end, optical filter and coupler, is employed to split the signals to its various components and is fed to the detecting element. The detector is subjected to several noise which degrade the received signal. The discussion on the types of noises at the detection is made and an equivalent mathematical model is constructed to simulate the receiver section of the network.

In Chapter 6 step by step description of the simulation set up has been provided. The simulation result and discussion has been provided in this section. Both the time domain and frequency domain simulation results are presented followed by some descriptive detail. The BER plots are provided and the comparison between the two models is also discussed in this chapter.

Finally, the conclusion on the project and future insight is presented in Chapter 7 . 


\section{Chapter 2}

\section{Fiber To The Home}

\section{$2.1 \quad$ FTTH, Technology Overview}

The ever growing demand for bandwidth by individual users and businesses energized the search for a means to provide reliable and cost effective high data rate transmission alternatives. The traditional copper based service to the "last mile"of the service can't provide the band width demand for the baseband services of the audio, internet and video. The pressure on the demand side for more band width is rapidly growing to exceed the capacity either the copper-based or wireless system can provide. Optical fiber cables have been the major transmission media in the range of long haul(hundreds and thousands of kilometers), in the metro area links(tens of kilometers) and in the interoffice aggregations of several kilometers. Now it also becomes a better candidate to be considered by researchers and engineers for the last mile of the service.

The access represents the portion of the network system between the central office or cable head to the subscribers premises. Currently, it is the only part of the fixed communications plant that has not already evolved completely into fiber. However, fiber optics is growing to become the dominant media in the access range of the last mile of users destination. The following figure shows the four layers of the optical fiber network in the transport layer of communication system and associated switching devices.

Traditionally the baseband signals such as cable TV, home phone and internet 


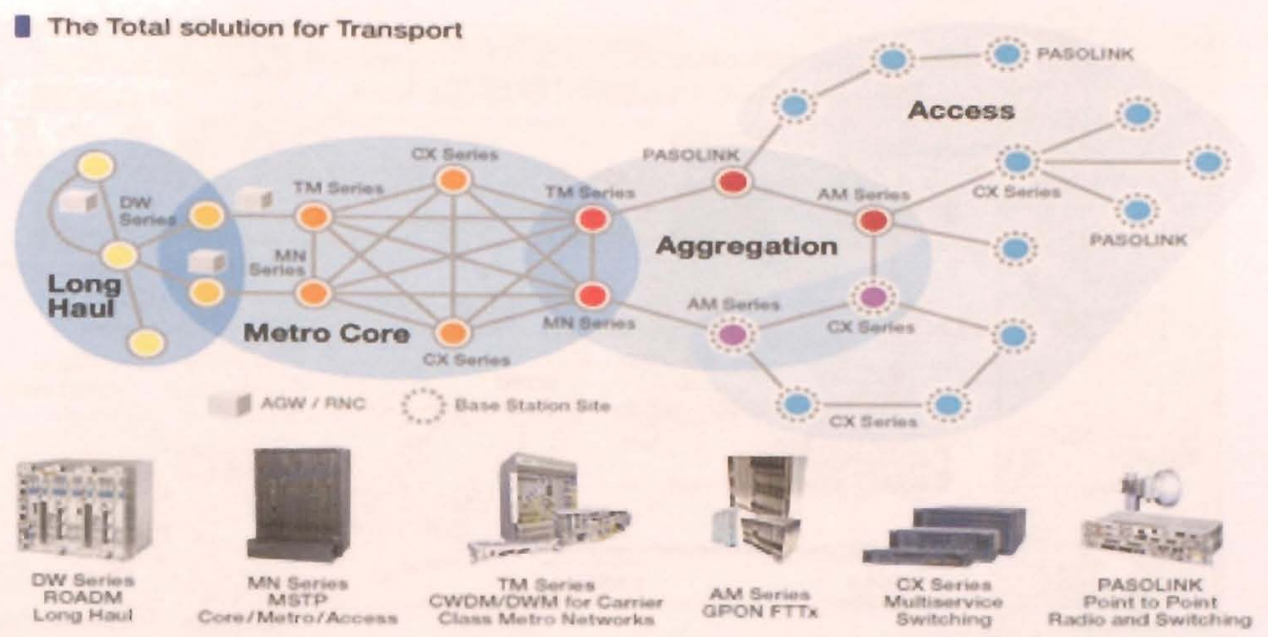

Figure 2.1: Schematic representation of different layers of the fiber optic network system [29].

service have been provided with the copper wire either with coaxial cable or twisted wire pair. With the increased services and the increasingly high demand of the tripe plays, these traditional channels could no longer support the data rate requirements of users. In order to make use of large band width capability optical fiber, basebands services of cable TV, internet and home phone are now provided by fiber optic networks through FTTH system. Therefore, FTTH is one of the highly evolving technology deployments in the communication system. FTTH represents the portion of the optical fiber link that extends from the central station to the home or business of the user. Once the optical signal arrived at the junction boxes of the wall at the home, or the base of the building, the message can be further transmitted by several other means such as coaxial cable or twisted wire pair. FTTH is the extension of the earlier fiber optic system deployments such as Fiber To The Curb (FTTC), Fiber To The Premises (FTTP) and Fiber To The Building (FTTB). Figure 2.2 depicts an example of the technology and service provision of FTTH by NEC corporation.

There are obvious reasons that some service providers and cable operators wouldn't completely agree and respond quickly towards development and deployment of FTTH. The companies which have invested fortunes in the copper infrastructure and are expecting returns in extended time, strive to earn their return before low cost optic fiber extended its services to the door of every home. However, most companies are at the 


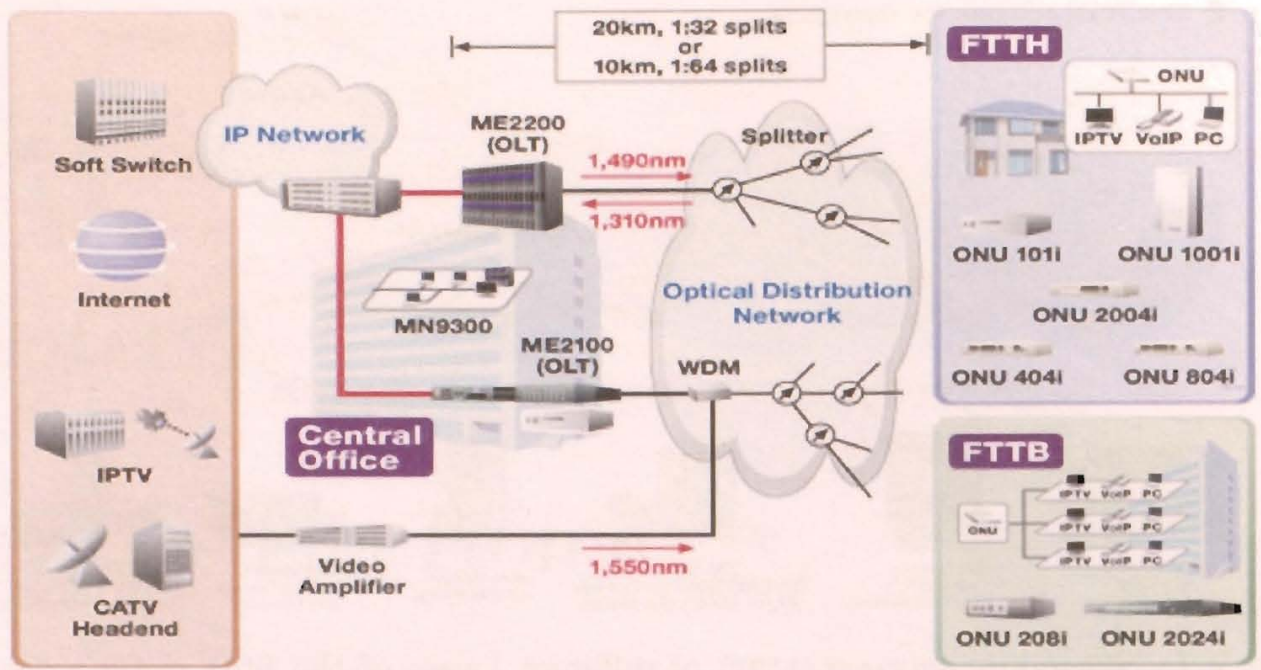

Figure 2.2: A pictorial representation of the major services provided by FTTH and its interconnection [29].

same page regarding using fiber optics in the access network for wireless backhaul, Greenfield residential, rural and remote areas. The growth of FTTH imposes series pressure for the predecessors technologies. The rate of growth of other technologies is greatly challenged by the fast growth rate of FTTH users. The diagram in Figure 2.3 compares the rate of increase of users in FTTH and ADSL at the early stage of FTTH deployment in Japan.

There is no doubt that the age of FTTH has arrived. The fits and starts of the past are over. In north America every major Telecom provider and small service providers are proceeding to deploy the trails of fiber predominantly PONs. The key to the realization of FTTH is the standardization of PON, allowing component vendors and system venders to focus on single solution [17].

\subsection{PON Systems}

Passive Optical Network(PON) is a point-to-multipoint (P2MP) network architecture which has single Optical Line Termination (OLT) at the central office end and several(usually 16 to 128) Optical Network Terminals (ONTs) at the consumer side. PON 


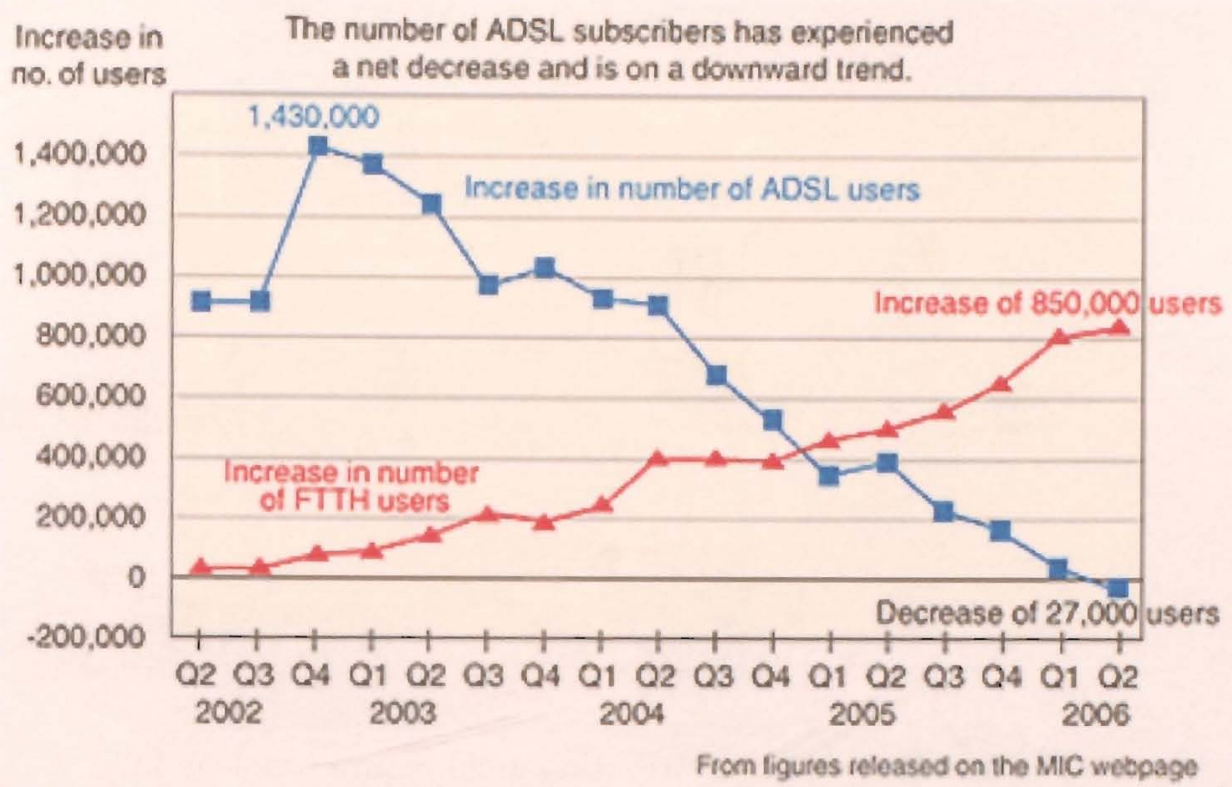

Figure 2.3: Comparison of subscriber growth rate in competing technologies of ADSL and FTTH in Japan [34].

has no active elements and doesn't need external power input for the optical splitters. The interior components of PON consists of passive splitters, splices and interleaving fibers. Since it doesn't need additional external power, it reduces the deployment and operation investments and provide comparative cost advantage over Active Optical Network (AON). Many researcher and service providers view PON as attractive solution to the first mile(last mile) problem. Compared to point-to-point (P2P) architecture, PON also reduces the number of optical transceivers and size of the optical fiber material required between the center of the service and the users terminal. PON can provide the major baseband services of data, voice, and video to a length of up to $20 \mathrm{~km}$ between central offices to consumer's location.

Since the message signal for several consumers is broadcasted through a single shared optical fiber, the transmission may be subjected to unintended listener. However, several techniques such as encryption could be employed in order to protect the downstream user's message from such eavesdropping. The uplink signal can be combined into the single fiber line by means of one of the several access protocols such as Time Division Multiple Access (TDMA). 


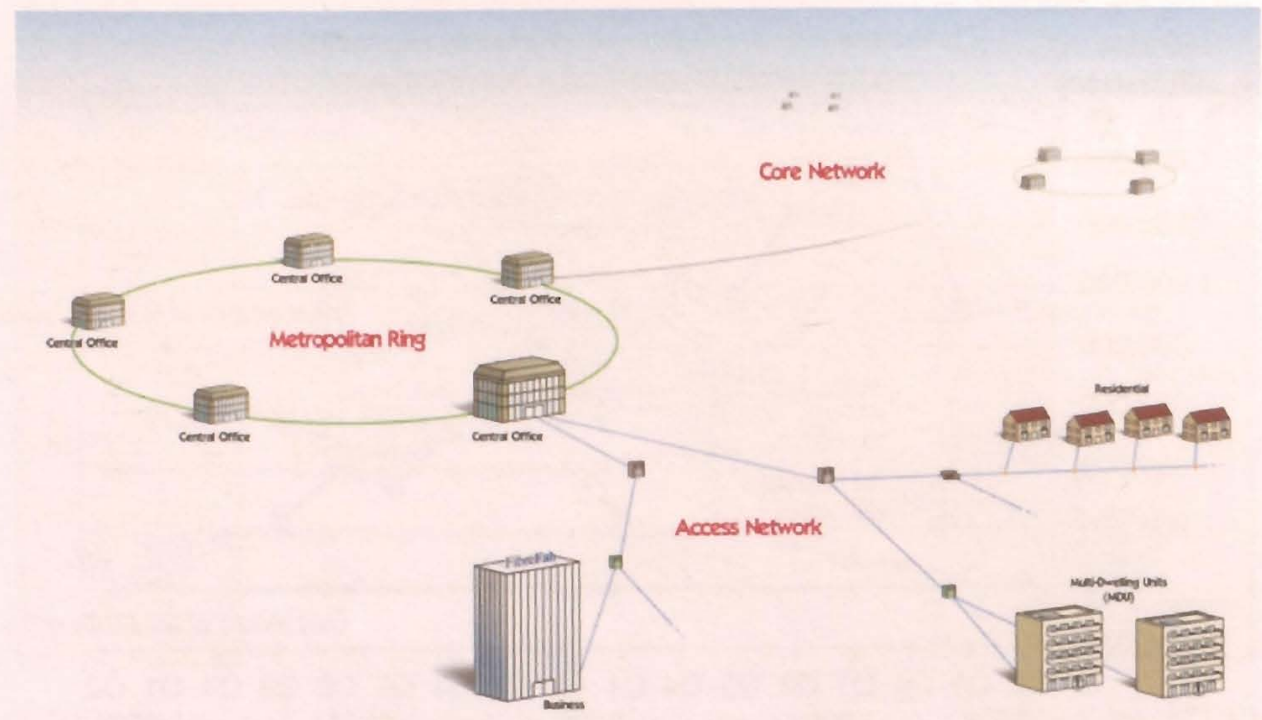

Figure 2.4: PON distribution architecture topology [35].

Based upon the interconnection architecture between the central office's OLT and several end users points, there are three common types of network topologies used in PON. These are ring PON, star(tree) PON and bus PON. They vary in performance characteristics, reliability, cost, ease of deployment and access for expansion. Due to the difficulty of low-perturbation optical-tap for efficiently coupling the optical signals, bus $\mathrm{PON}$ is relatively difficult to implement. The optical information is accessed passively tapping off the optical power using passive coupling elements which do not employ electronic components. In the case of ring PON topology, the successive service terminals are linked each other with P2P connections and create a single closed path. At each node it requires an active device to identify its address in order to accept the arriving information whenever the message is destined to this node. In star PON topology all the nodes are connected to one hub called the central office node. Figure 2.5 shows the three main kinds of PON. 


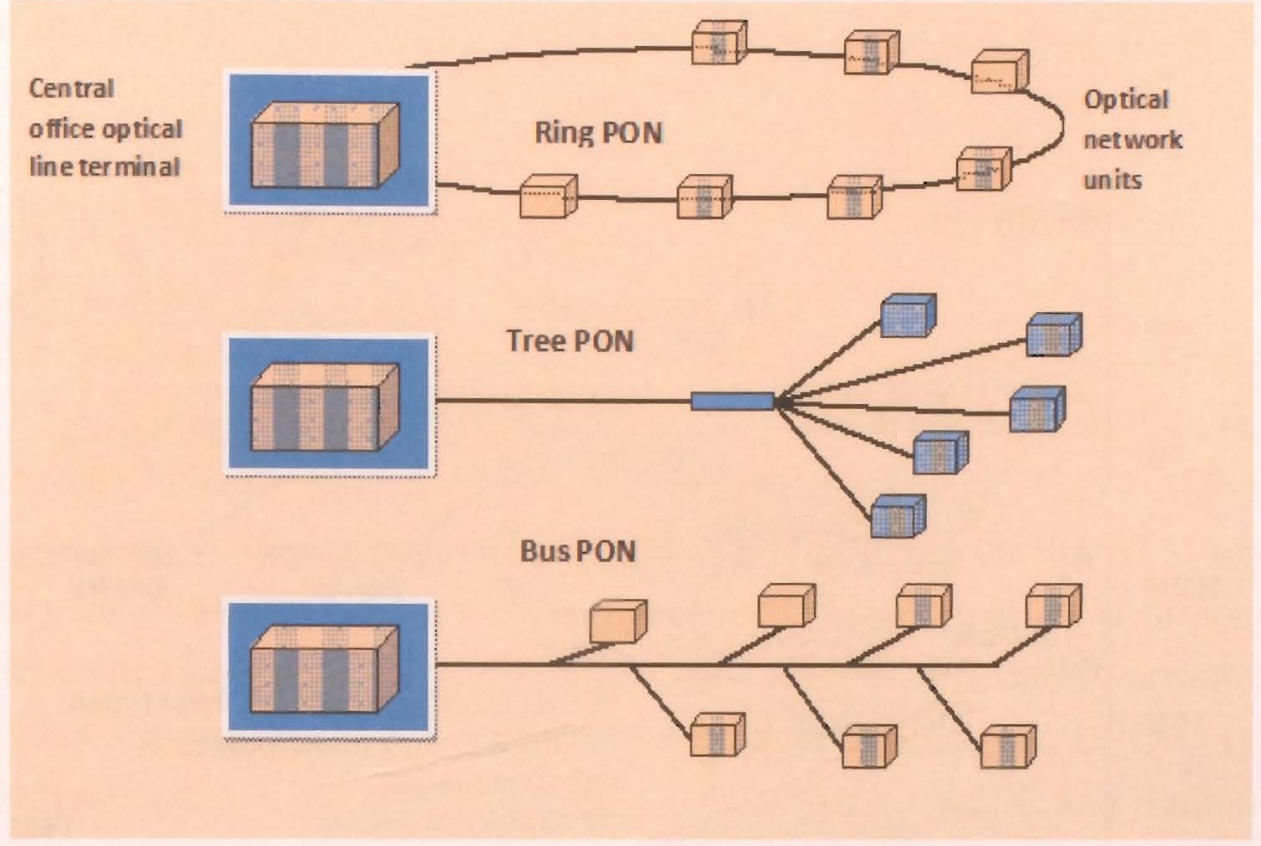

Figure 2.5: Three major kinds of PON topologies.

\subsection{PON Standardization; IEEE \& ITU-T}

Standardization is a key factor in all kinds of technologies so that all the researchers, service providers, producers, venders and consumers speak the same language. The two major standardization institutes in the fiber optics communications and PON are IEEE (Institute of Electrical and Electronics Engineers) and ITU (International Telecommunications Union). The development of PON from the early 1990's to 2011 brought about the fastest rate of information exchange and evolved to different standards. In late 1990's a group of operator called Full Service Access Network (FSAN) formed with the objective of "Global Domination of the Fiber Access". Later this group submitted the responsibility to ITU. ITU-T released standards such as GPON (G.984x2003) with the capability in the gigabits range. The IEEE version of the standard is termed EPON (Ethernet-PON) and identified by the version such as IEEE 802.3ah 2004. Both organizations produced their own standards up to 1 gigabits or 10 gigabits however the standards are mutually incompatible. Generally, PON rates grow from $155 \mathrm{Mbits} / \mathrm{sec}$ up to 2.4Gits/sec and projected to the new generation PON of 10Gbits/sec by 2012 . The following figure shows the general evolution and deployment of both standards over 
time.

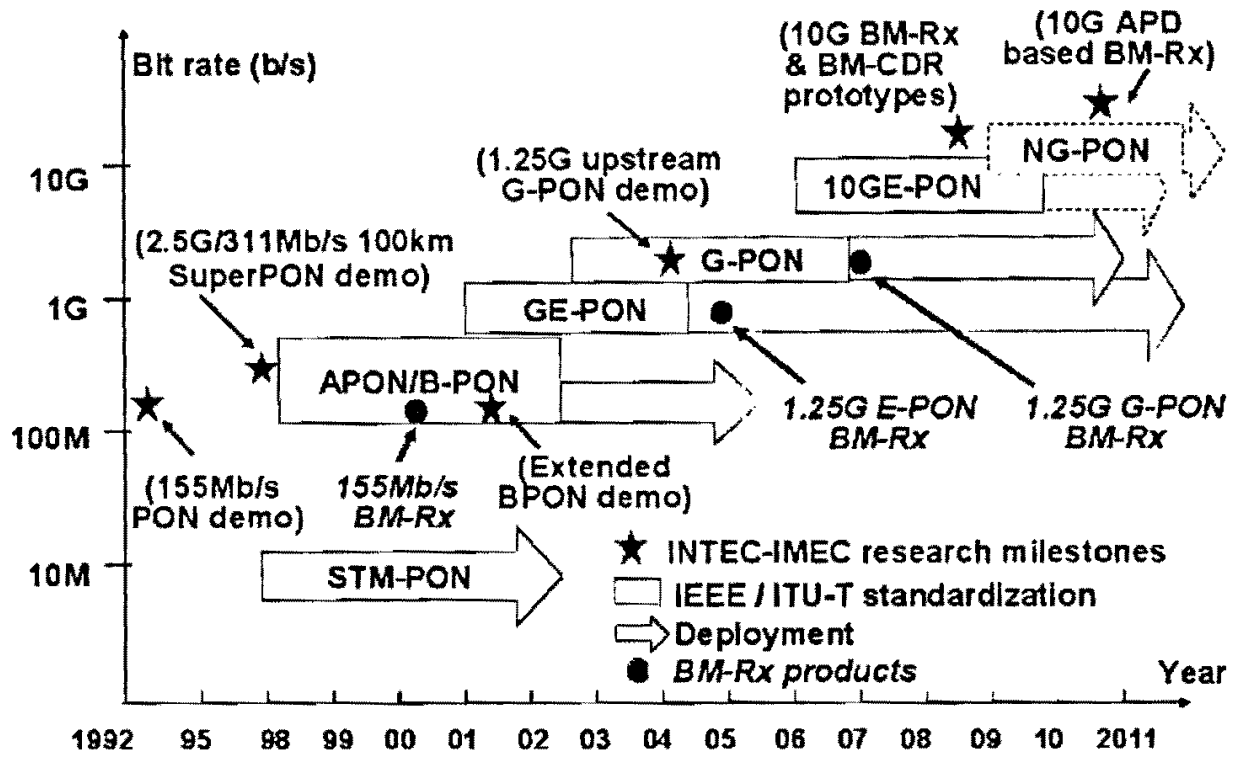

Figure 2.6: Pictorial representation of PON standard's timeline and their deployment [36].

\subsection{EPON/GEPON}

IEEE is the largest standardization institute for PON technology. 802.3 EPON (Ethernet $\mathrm{PON}$ ) is one of the standards provided by IEEE. Standards vary and are specified by their provision of data in upstream and downstream direction. EPON provides a symmetrical frame of $1 \mathrm{~Gb} / \mathrm{s}$ in both upstream and downstream directions. It is the most widely deployed format of PON. Currently, there are over 40 million installed EPON ports making it the most widely deployed PON technology globally[31]. EPON is also extensively used for business services as part of the Data Over Cable Service Interface Specification (DOCSIS). It is the basis for the service provider's and cable operators' provision of DOCSIS Provision of EPON (DPoE) specifications. EPON can be implemented either in only data centric services or fully fledged data, voice and video provision networks. IEEE completed the Ethernet First Mile project in 2004 and as part of it provided (GEPON) the $10 \mathrm{Gbit} / \mathrm{s}$ EPON or $10 \mathrm{G}$-EPON provision versions PON in IEEE 802.3av. The list of some selected standards and their specification is 
provided in Table 2.1 .

\subsection{APON/BPON/GPON}

ITU is the first to provide passive optical network standards. Since then, several version of ITU-T standards has been released with increased performance and data rate. Some of the standards include ITU-T / G.983, ITU-T /G.984, ITU-T /G.987. APON (ATM PON) is the first PON standard to be released. It was based on the ATM system and its primary usage was for business purposes. With the decline of ATM protocol, ITU$T$ has improved the original APON standard and provided the later version of ITU-T G.983. This standard commonly referred as broadband PON, or BPON. It is based on APON technologies and standards, however it supports WDM and dynamic higher upstream bandwidth allocation. A typical APON/BPON provides $622 \mathrm{Mb} / \mathrm{s}$ (OC-12) of downstream bandwidth and $155 \mathrm{Mbit} / \mathrm{s}$ (OC-3) of upstream traffic. Table 2.1 shows the comparison of different standards and their capacities.

The further improvement in the ITU-T standardization process provided the evolving of GPON(Gigabit PON). GPON evolution is Based on BPON, however it shares several similarity with GEPON. As the primary motive of the standards evolution is their capacity to support higher data rate, GPON carries higher data rate and has enhanced data security features. The original intention for deployment of GPON was to widen the support and acceptance of technology which can support multiple protocol, however, the added complexity was a challenge. A key characteristic of GPON is the 2.5 Gbps downstream and 1.25 Gbps upstream data rate [34]. GPON operates in a very similar fashion to GE-PON when supporting Ethernet as its primary transport protocol. But since Ethernet, gigabit Ethernet and 10Gb Ethernet do not support a $2.5 \mathrm{GHz}$ clock rate, unlike GE-PON, GPON does not benefit from the availability of low-cost Ethernet optical components. The ITU-T G.984 (GPON) standard represents a boost, compared to BPON, in both the total bandwidth and bandwidth efficiency through the use of larger, variable-length packets [34]. Again, the standards permit several choices of bit rate, but the industry has converged on 2.488 gigabits per second (Gbit/s) of downstream bandwidth, and $1.244 \mathrm{Gbit} / \mathrm{s}$ of upstream bandwidth. GPON 
Encapsulation Method (GEM) allows very efficient packaging of user traffic with frame segmentation. 10G-PON has $10 \mathrm{Gbit} / \mathrm{s}$ downstream and $2.5 \mathrm{Gbit} / \mathrm{s}$ upstream framing is "G-PON like" and designed to coexist with GPON devices on the same access-network technologies are clearly desired for next generation broadband applications [34]. The following table revises the major PON standards and their bite rate capacity in upstream and downstream directions.

Table 2.1: Comparison of different standards for PON.

\begin{tabular}{|c|c|c|c|}
\hline & Network base & Standard & Bit rate \\
\hline EPON & 802.3 ah 2004 & IEEE 802.3 & $1 \mathrm{~Gb} / \mathrm{s}$ (downlink) \\
\hline (Ethernet Protocol) & & & $1 \mathrm{~Gb} / \mathrm{s}$ (uplink) \\
\hline GEPON & 802.3ah 2004 & IEEE 802.3 & $10 \mathrm{~Gb} / \mathrm{s}$ (downlink) \\
\hline (Ethernet Protocol) & & & 1Gb/s(uplink) \\
\hline 10GEPON & 802.3 ah 2004 & IEEE 802.3 & $10 \mathrm{~Gb} / \mathrm{s}$ (downlink) \\
\hline (Ethernet Protocol) & & & $10 \mathrm{~Gb} / \mathrm{s}$ (uplink) \\
\hline APON & G.983 & ITU-T & $622 \mathrm{Mb} / \mathrm{s}$ (downlink) \\
\hline (ATM based) & & & $155 \mathrm{Mb} / \mathrm{s}$ (uplink) \\
\hline BPON & G.983 & ITU-T & $622 \mathrm{Mb} / \mathrm{s}($ downlink $)$ \\
\hline (Broadband PON) & & & $155 \mathrm{Mb} / \mathrm{s}$ (uplink) \\
\hline GPON & G. $984 \times 2003$ & ITU-T & $2.5 \mathrm{~Gb} / \mathrm{s}($ downlink $)$ \\
\hline (Multiple Protocol) & & & $1.25 \mathrm{~Gb} / \mathrm{s}$ (uplink) \\
\hline $10 \mathrm{GPON}$ & G.987 & ITU-T & $10 \mathrm{~Gb} / \mathrm{s}$ (downlink) \\
\hline (Multiple Protocol) & & & $2.5 \mathrm{~Gb} / \mathrm{s}$ (uplink) \\
\hline
\end{tabular}




\section{Chapter 3}

\section{Radio over Fiber}

\subsection{RoF Basics}

Radio over Fiber (RoF) is currently evolving attractive technology by which a radio signal is made to modulate optical carrier signal and transmitted through optical fiber. This technique enables the transmission of microwave and millimeter waves to be carried through a guided optical fiber instead of open wireless media. RoF facilitates wireless access by exploiting the large bandwidth capacity of optical fiber and limiting the number of expensive wireless parts required. Moreover, RoF provides a feasible and better option to install smaller antenna in some inaccessible and confined areas, such as underground facilities and metro tunnels. All sort of radio signals including cable television, broadcast or satellite base station signals can be carried through optical fibers networks; however, the term RoF particularly refers to optical link which are applied for wireless access.

Currently, the traffic on Wireless Local Area Networks (WLAN) has grown tremendously as its provides easy access to high-speed internet services. The deployment of WLAN has been increased in oredr to cope with the high demand in the wireless services. However, with increasing coverage area, increasing number of subscriber per area and increasing bandwidth demand per subscriber, WLAN alone cannot satisfy the created wireless services demand. The increased deployment of WLAN is also subject to several technical and financial drawbacks. First of all, radio equipments have high installation, operation and maintenance cost. Moreover, since wireless accesses are routed 
through unguided media, they are subjected to series RF interferences. Eavesdropping is also a series issue for services in the wireless domain unless a measure is taken to prevent it. Channel allocation, frequency spectrum administration, and existence of several WLAN standards are also the shortcoming for wireless services. Therefore, the WLAN becomes a more complex service, which requires high standard and high cost in design, administration and maintenance .

Several technologies have been suggested to cope with the ever growing demand in wireless services and to eliminate the drawbacks inherent to it. RoF is one of the promising candidate to help and facilitate the radio accesses. RoF uses fiber optic channel and light wave to transmit radio signals to long distances. It is also convenient to access some blocked spaces that would have been harder to reach with wireless means. In RoF the radio signal are carried away and distributed using optical components and associated technologies. The central station (CS) and the remote access node (RAN) in RoF system are connected by means of optical links.

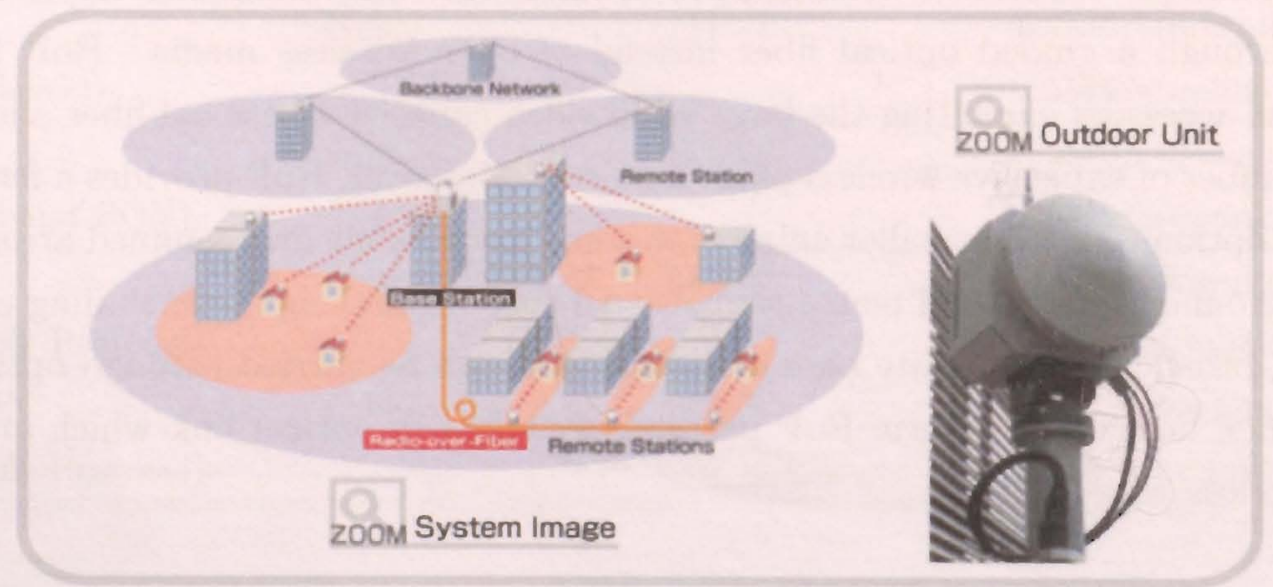

Figure 3.1: Pictorial representation for millimeter wave broadband wireless access system with Radio over Fiber application [30].

In RoF the electrical domain radio signal from its source could be the modulated RF signal, modulated IF (Intermediate Frequency) signal or even baseband signal. This electrical based radio signal again modulate the optical carrier signal using optical modulation techniques. Following this modulation the signal is in light domain until it reaches its remote destination and is converted back to electrical based signal. While 
it is carried through the optical fiber channel, the signal is light wave and is subjected to all kind of alteration and deformation that occurs in optical domain signals.

It is a fact that the futuristic direction in wireless network heavily implement RoF for both microwave and millimeter wave radio bands. In order to achieve higher capacity the DWDM can also be implemented with RoF system. Above all the prime motive is to carry large wireless signals and transport them in optical form between a central station and a set of base stations. The radiation of the signals takes place at small base station with limited radio range.

It is customary to classify RoF systems into two RF-over-Fiber and IF-over-Fiber depending on the range of the radio frequency range to be carried. Usually, RF signals greater than $10 \mathrm{GHz}$ are termed as RF-over-Fiber. Since the signal is relatively high already, there need no frequency up/down conversion for imposing the signal on the light carrier or to radiate to the air at the base station following conversion of the light signal to electric domain signal. In the case of IF-over-Fiber architecture, the radio signal is less than $10 \mathrm{GHz}$ and frequency up/down conversion might be required to provide enough separation in modulation and provide required frequency range for transmission.

\subsection{Advantages of Radio over Fiber}

\subsubsection{Low Complexity}

The Remote Station (RS) in RoF mainly consists of an optical-to-electrical (O/E) conversion devices. The additional devices demanded at RS are some amplifiers, frequency converter, and the antenna. The major radio units in signal generating, controlling and resource management circuitry are moved to central station. Several RS share this radio unit at the central station. Since a high frequency radio signal can travel through the fiber, optical link of RoF avoids the necessity of generating a radio signal at RS. Therefore, the design of a network system with RoF is less complex. The remoteness of the antenna unit from easy access also has other technical benefits. Generally, the most important advantage of RoF system is its provision to move the highly expensive 
base station and antenna units to the central office and make use of simple and less expensive antenna unit at remote stations.

\subsubsection{Low Attenuation}

The rate of attenuation of signal varies from media to media. Generally, signals transmitted through optical fiber attenuate about $0.2 \mathrm{~dB} / \mathrm{Km}$. The rate of attenuation for optical fiber is much lower than the copper wire competitors or even wireless media. Applying optical fiber therefore provides a system which has less number of repeater stations as the signal can travel longer distance before its strength fall to some minimum value which requires boosting.

\subsubsection{Low Cost}

Fiber optical networks need simple structure for base stations, and have low power consuming and easily maintainable devices. RoF needs less number of radio units at remote stations and all the major radio stations are moved to central station. The overall effect of these is that RoF provides lower cost of infrastructure and deployment, reduction of power consumptions as well as device maintenance cost. Simpler and easier system also reduces the design, installation and operation costs.

\subsubsection{Future Proof}

The highly pronounced advantage of fiber optic is its high data rate capacity. Lately there are reports claiming that optical fibers can transmit data in the range of $\mathrm{Tb} / \mathrm{s}$ (Tera Bits per second). The direction of the future shows that wideband wireless service is a must. Therefore fiber optics systems are the solutions for the high speed demand of the future generation networks. Moreover, protocol and bit-rate transparent technologies can easily be adopted for RoF which makes it higly applicable for the current as well as future application. 


\subsection{Applications of Radio over Fiber}

The most important application of RoF is for places where their geographic orientation causes difficulty to provide coverage by the conventional ways. These areas include enclosed structure such as shopping malls and metro subways, naturally obstructed areas like mountainous locations and jungles, as well as isolated and blocked sites such as miners tunnels. Covering these zones with the regular wireless backhaul link is either impossible or extremely expensive beyond justification. The optical fiber link provides easier access to such places. In addition to the easy access the optical link provides additional advantages such as low line losses, less susceptibility for lightening strike, and increased simplicity.

The other important application of RoF is in places where there is congestion of wireless users. Because of the limited nature of the RF spectrum as a resource for wireless applications, in places where large number of subscriber are located in very small area, like in some down town locations, a point is reached where the cellular system fails to serve all users. In such situation low antenna and small cell size such as Pico and Micro cell structure can be solutions and RoF is used for cell optimization of these situations. Moreover, lesser radiation from the low antenna power means a safer system for health, and even low power system also reduces the power consumption of the mobile devices and provide a longer battery life. 


\section{Chapter 4}

\section{Hybrid Fiber Optic Services}

The hybrid FTTH and RoF is a newly developing idea of delivering the FTTH baseband services of cable TV, wired telephone and internet integrated with the emerging RoF wireless services into an interleaved access network. This is conceived and developed by integrating the two signals and simultaneously transmitting them at the same optical carrier frequency into the fiber optic networks so that they share the same infrastructure. The prime motive behind converging these two systems is the ever increasing demand for broader bandwidth in both services. For the radio system the RoF has been already a promising method in providing a better wireless access in a larger and distributed area. The future direction is shown to require more and more services in the triple play in both wired and wireless domains. As the services are expanding in both coverage and density, the bandwidth limit happened to be the bottle neck for the communication network. Hybrid FTTH and RoF is a promising option in using the extremely huge bandwidth potential of optical fiber networks.

The basic idea of hybrid FTTH and RoF is to be able to interleave, modulate and transmit the baseband signal of the wired services and the radio frequency (RF) of the wireless services into a single wavelength of an optical fiber channel. Integrating the services provide an effective means to reduce the extra infrastructure and deployment investment as well as subsequent maintenance and operational costs. Good design and careful analysis are required in order to obtain a cost effective as well as efficient system. In recent days several papers have been published to demonstrate the simultaneous modulation and transmission of these two signals [1]-[3], [9], [12]-[14]. 
In this project two distinctive architecture for the design of the integration of two optical based service signals have been developed. The models and their schematic diagrams are shown in Figure 4.1 and Figure 4.2. The implementation of the simulation for the modulation, transmission and detection of the hybrid signal have been developed in the subsequent sections. The two approaches are identical in the transmission and detection sections. However, the modulation of the first case (see Figure 4.1) is done in such a way that the two signals are first shifted to their respective subcarrier frequency ranges and then mixed in the electric domain prior to applying them to the MZM modulator. The integrated signal then fed to the single port of the $\mathrm{Y}$ branch MZM in order to modulate the optical carrier with the hybrid signal. In the second option (see Figure 4.2), after the two signals are shifted to their subcarrier frequencies, they are applied to the two different ports of the MZM modulator, so that the optical carrier is modulated by each signal subcarrier and the two signals get mixed in the optical domain following the MZM. In these figures the electrical domain paths and processes are indicated with dashed line while the optical stages and paths are represented by a solid line.

The tunable laser is the optical carrier source providing a low-phase noise super continuum (SC) light with $1550 \mathrm{~nm}$ wavelength. The light is directly fed to the Y-branch MZM and made to pass through the two branches of the modulator. A random word generator simulate the first service 1 source generating randomly distributed sequence of bits at the rate of $622 \mathrm{Mb} / \mathrm{s}$. This simulated data is mixed with a low power noise generated from the band-limited white noise source to simulate the noise persistent to radio system. The band-limited white noise doesn't represent the practical equivalence of the noise but it closely approximates the noise in the radio signals. This signal is further modulated with a $25 \mathrm{GHz}$ sinusoidal subcarrier frequency to up convert the radio to the upper frequency range and provide a sufficient frequency range for interleaving of the second service signal in lower frequency range. Another random word generator simulate the second service signal source simulating randomly distributed sequence of bits at the rate of $1.25 \mathrm{~Gb} / \mathrm{s}$. This digital word sequence is further modulated with a $5 \mathrm{GHz}$ sinusoidal subcarrier frequency to up convert the baseband range and accommodate the simulation for the MZM model. 
The two signals are either mixed together and fed to MZM in order to modulate the SC light source in case of one leg option (Figure 4.1) or fed to different legs of the Y-branch MZM to modulate the optical carrier separately and get mixed in the light domain of the other end of Y-branch MZM (Figure 4.2). The signals in the MZM are subjected to non-linearity effect of the modulator. In order to simulate this, the signal in the simulation are set to be small enough not to drive the MZM out of the linearity range. The output of the MZM can be amplified as to accommodate the transmission losses and detector sensitivity. The models for the MZM modulator, the transmission channel and the detecting devices are developed using the mathematical model that will be developed in Chapter 5 . This chapter discusses the theoretical basis and optical elements of the design in detail. 


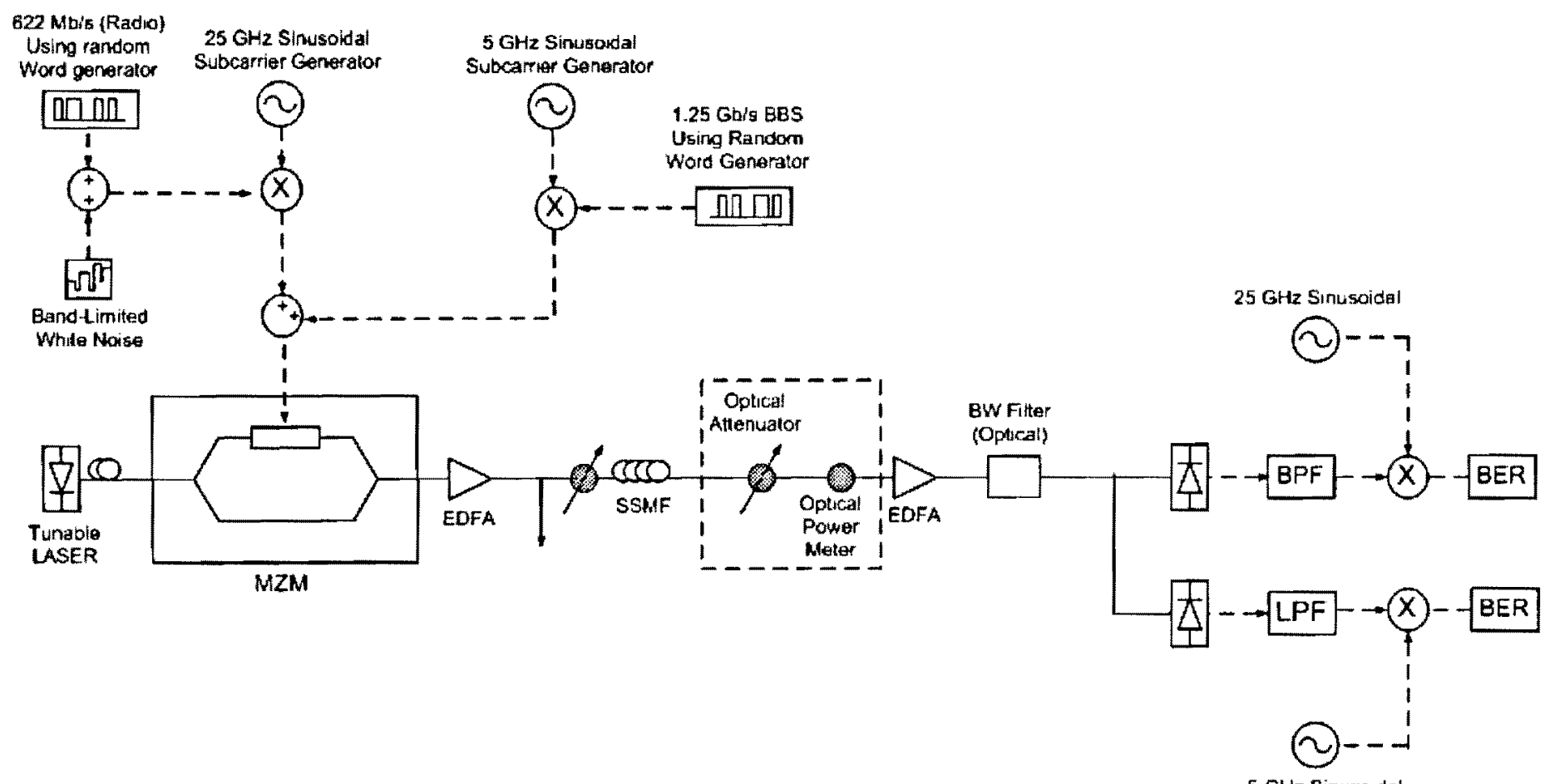

$5 \mathrm{GHz} \operatorname{Sinusordal}$

Figure 4.1: The schematic diagram for the hybrid signal of two RoF services using single leg MZM. The two signals mixed in the electric domain and fed to the single leg of the MZM. The broken lines depict the path of electrical signal and processes and it is done in Simulink for simulation. The solid lines represent the optical stages of the link and processes are simulated in MATLAB. Notice that the MZM has only one port and the electrical domain signals are mixed before they come to the MZM port. 


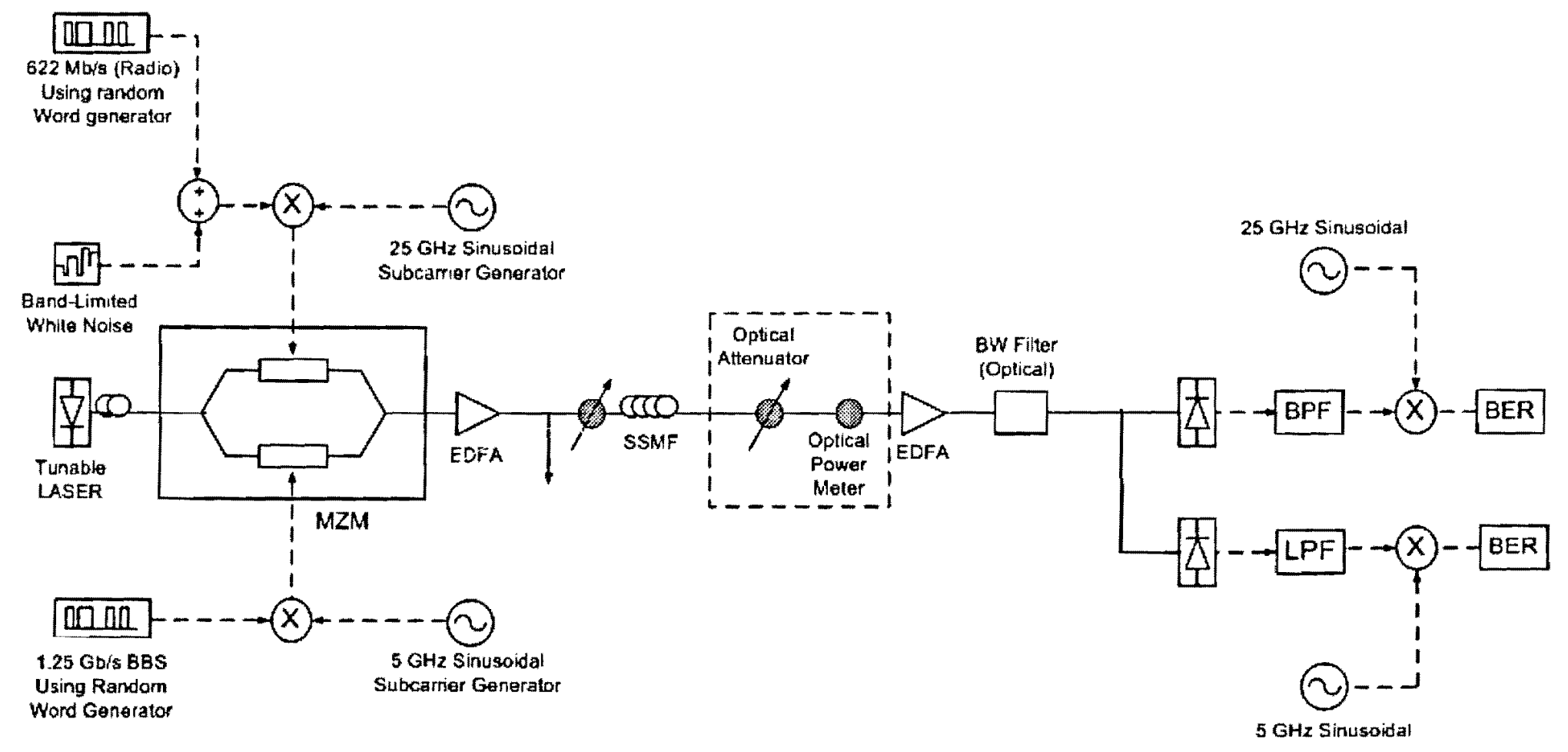

N

Figure 4.2: The schematic diagram for the hybrid two RoF services using two leg MZM. The two signals mixed in the optical domain. The broken lines depict the path of electrical signal and processes and it is done in Simulink for simulation. The solid lines represent the optical stages of the link and processes are simulated in MATLAB. Notice that the MZM has two separate ports and each electrical domain signal goes directly to each port. 
The simulation set up is done to understand and develop the the feasibility of the hybrid network. The laser output power is capable of providing the power that can be detected at the receiver end. The $622 \mathrm{Mb} / \mathrm{s}$ and $1.25 \mathrm{~GB} / \mathrm{s}$ signals which simulate the two service signal are generated by arbitrary digital waveform generator of Simulink modules. The subcarrier sinusoidal are used to up convert the digital signal to a convenient range for simulation and provide sufficient spectral separation between these two signals. The optical carrier is set to be at $1550 \mathrm{~nm}$ wavelength in both cases of the architecture. The power loss due to attenuation is about $5 \mathrm{~dB}$, and the estimated insertion loss for MZM is about 14dB [9]. Simulation of EDFA is used to compensate for these loses within the optical domain.

The two different architectures are designed in order to compare the difference in modulation of the two signals. The mathematical model and its derivation are presented in the next section. The two kinds of MZM are also shown in Figure 5.1 and 5.2 of the next chapter. The biasing of the MZM is assumed to be at full point providing the modulation index $\left(M I=V_{p-p} / 2 V_{\pi}\right)$. Setting this value to 1 will maximizes the undesired sideband suppression ratio. The transmission channel is considered for its attenuation and dispersion effects of the fiber optic materials. Again the equivalent mathematical derivation and model for simulation will be provided in the next chapter. At the receiver end, optical filters and couplers are employed to split the signals to its various components and is fed to the detecting element. The detector converts the signal to electrical current and feeds it back to the low pass and band pass filters connected to its end. The detector is subjected to several noise which impair the detectability of the received signal. The detail discussion on the types of noise at the detection is provided in the next chapter. This discussion is also the base to construct and simulate the receiver section of the network.

The received electrical signal is mixed with the original sinusoidal subcarrier frequencies to detect and demodulate the original word sequence of each service. The BER curve is constructed in order to compare the performance of the two architectures in consideration. The signal in the first model with single leg MZM would have higher Vpp voltage at some points as the two signals are added in the electrical domain. This will derive the MZM out of its linearity range and some messages could be distorted. 
Otherwise model 1 might require MZM with higher bias voltage. The second model might solve this problem, however with regard to cost, it might not be the preferred choice. The complete discussion, performance evaluation and comparison will follow in the subsequent chapters. 


\section{Chapter 5}

\section{Basic Theory and Mathematical Models}

\subsection{Modulation Model}

In communications based on optical networks the electrical signal has to be modulated over the optical carrier in order to be carried on through the optical fiber links. Several methods have been developed to modulate the electrical message into the light wave. The direct current modulation techniques have been successful by which the input current for the laser is changed with the modulating signal. Time independent optical intensity can be produced by modulating the laser around its bias current. Direct current modulation is subjected for several non-linear phenomena. Intersymbol Interfernce ISI is a consequence of these non-linearity. Other limitations such as transient oscillation and distortion of the output light pulse also broaden the signals optical spectrum, and therefore increase BER. Generaly, directly modulating the current deriving the laser diode causes; turn on delay, relaxation oscillation, mode hoping, chirping of optical wavelengths.

In order to solve problem associated to direct modulation several suggestion are put forward. Some suggestions are to solve the problem of direct modulation. Some of these suggestions are modification of the physical laser, the use of external optical feedback, external electronic circuits and shaping of the modulation current. More- 
over, direct modulation has undesirable effect on wavelength modulation which affects system which employs WDM techniques. Direct modulation of laser becomes almost impossible at data rates above $10 \mathrm{Gbps}$. Therefore in order to increase such a bandwidth application it is possible to take the advantage of external modulators. External modulators allow higher bit rate and there is no direct interaction between the information being applied to the modulation and the internal dynamics of the laser. Thus to extend the bandwidth capability of single-mode fiber systems there is a requirement for highspeed modulation which can be provided by IO waveguide intensity modulators [10]. A basic OOK (on/off keying) modulator can be implemented using simple techniques on switches and active beam splitters. An important waveguide modulator is based upon a Y-branch interferometer which employs optical phase shifting produced by the electro-optic effect.

Since hybrid optical systems require high data rate and travels very long distance, external optical modulators is necessary. External modulators used in Optical communication are primarily based on Electro-optic effect, magneto-optic effect, and acoustooptic and electro-absorption effect of semiconductors. Mach-Zehnder Modulator (MZM) using LiNbO3 is a popular choice for implementation in high speed optical networks.

\subsubsection{Pockel's Effect}

The electro-optic effect is the change in refractive index that occurs in a noncentrosymmetric crystal at the presence of an applied electric field. MZM exploits the property of electro-optic effect. The electro-optic effect can be of two types. The first one is the Pockel's Effect by which the change in refractive index is linearly proportional to the applied electric field. The second electro optic effect is termed as Kerr Effect. In this case, the proportionality between the refractive index to the applied electric field is quadratic. In other words, the refractive index change with square of the voltage applied. The change in refractive index due to Kerr Effect is negligible compared to the change produced by Pockel's effect. Optical modulator makes use of the principles of Pocke'effect. Lithium Niobate (LiNbO3) is the most widely used crystal to fabricate optical modulators because of its large electro-optic effect, small optical loss and good 
coupling efficiency. In LiNbO3, the change in refractive index due to Pockel's effect is given by,

$$
\Delta n=\frac{1}{2} n^{3} \gamma E
$$

where, $n$ is the refractive index, $\gamma$ is electro-optic coefficient of the crystal, and $E$ is the electric field intensity applied along the axis of the crystal. The change in the refractive index causes change in the phase of the light wave. Therefore the phase change is proportional to the applied voltage. The voltage needed to be applied in order to produce a phase shift of 180 degree is given by,

$$
V_{\pi}=\frac{d \lambda_{0}}{n^{3} \gamma L}
$$

where, $d$ is the distance between the electrodes, $\lambda_{0}$ is the wavelength in vacuum and $L$ is the interaction length. This voltage is termed as full wave voltage. The linear electrooptic effect is traditionally expressed as a linear change in the inverse refractive index squared:

$$
\Delta\left(\frac{1}{n^{2}}\right)_{i}=\sum_{i} r_{i j} E_{j} ; j=x, y, z
$$

where, $E_{j}$ is the applied electric field in $\mathrm{j}$ direction.

The crystal orientation is usually chosen so as to obtain the largest electro-optical effect. This means that if the applied electric field is along the $\mathrm{Z}$ axis, then the light polarized along $\mathrm{Z}$ see the largest field-induced change in refractive index.

Phase modulation is achieved by applying a field to one of the geometry. The field is roughly $V / G$ where $G$ is the gap between the two electrodes. For an electrode length $L$, the phase shift caused by the change in the refractive index exhibited by an electro-optic material with the application of an electric field can be given as in Equation 5.4 and 5.5 below. The phase change for light propagating in the material is given in relation to the applied voltage. This phase change $\delta \Phi$ is accumulative over a distance $\mathrm{L}$ within the material and is given by:

$$
\delta \phi=\Delta n_{Z} K L=-\frac{n_{0}{ }^{3}}{2} r_{33} \frac{V}{G} \Gamma K L .
$$


Substituting Equation 5.1 into the above equation gives:

$$
\delta \phi=\Delta n K L=\frac{2 \pi}{\lambda} \delta n L
$$

where, $\lambda$ is the wavelength of the light. When the electric field is applied transversely to the direction of optical propagation, we may substitute for $\delta n$ and get:

$$
\delta \phi=\frac{\pi}{\lambda} n_{1}^{3} r E L
$$

One of the considerations when designing a phase modulator is to reduce $V_{\pi}$ which could be done either by reducing $d$ (cross sectional dimension of the cut) or increasing $L$. However, the capacitance formed between the electrodes is directly proportional to $L$ and inversely proportional to $d$, hence reducing $V_{\pi}$ results in increased capacitance which slows down the operation of MZM modulator.

\subsubsection{Y-branch Interfrometeric Mach Zender Modulator (MZM)}

The Mach-Zehnder (MZ) interferometer is one of the most common applications of nonlinear optical devices. It allows a light wave to be either transmitted through the device or not by simply applying the half-wave voltage to introduce a phase change due to the electro-optic effect. There is a very high demand for practical polymer-based $\mathrm{MZ}$ devices not only because they reduce the required modulation voltage, but because they also allow for smaller, more compact devices, which can then be easily integrated into other applications [10].

The MZ Modulator is a relatively simple device based on the principles of the interference of light waves. A light beam is split into two equal parts, each with half the amplitude of the original beam, travels a certain length, and is then recombined. When no voltage is applied to the arm of the MZ, the path lengths the two beams travel are exactly equal, and when they recombine they interfere constructively to produce a beam with the original amplitude at the output. This is known as the "on" state of the MZ. For a MZ made of a nonlinear optic material, when the half-wave is applied to one arm of the MZ, it introduces a $\pi$ phase change in one of the beams. When the two beams recombine at the output, they now destructively interfere and thus no light 
passes through the device. This is known as the "off" state.

\subsubsection{Derivation of Modulation Equation for Single Signal Model}

The following section describes the step by step development of the mathematical models for single signal input (one leg model) modulation. For simplicity we first consider the amplitude modulation or On Off Keying(OOK) situation and then we will expand the discusion for general case. Let us assume an input electrical signal $u(t)$ is to modulate a light wave and is carried through an optical fiber link. $u(t)$ can be written as:

$$
u(t)=\alpha \sin (\Omega t)
$$

with small amplitude $\alpha$ and frequency $\Omega$. Let $\mathrm{y}(\mathrm{t})$ be the electric feild of the optical carrier light wave from super continuum laser source $\left(E_{i n}(t)\right)$ as indicated in Figure 5.1:

$$
y(t)=A e^{j \omega t}
$$

with amplitude of $A$ and optical frequency $\omega$. The Mach Zender modulator will result in

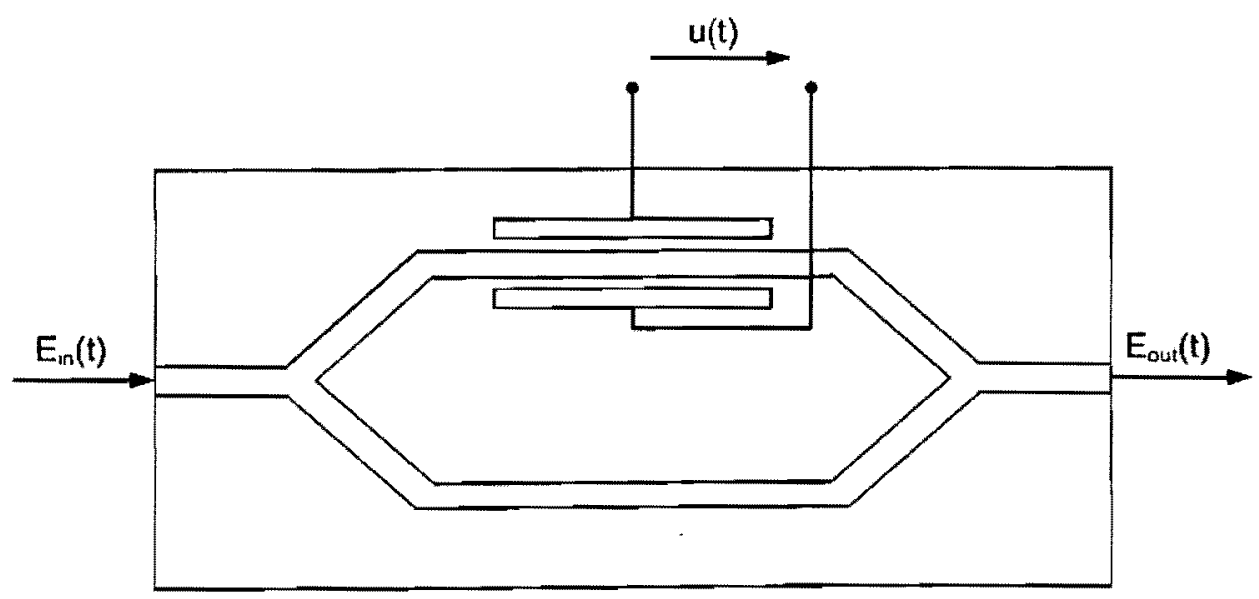

Figure 5.1: A structural representation of the one signal input $Y$ branch MZM

the phase shift of the optical signal in proportion to the applied sinusoidal modulating electric signal. Therefore, the modulated optical signal is written as:

$$
y^{\prime}=M(y(t), u(t))=A e^{j \omega t+j \alpha s i n(\Omega t)}
$$


where, $y^{\prime}$ represents the modulated optical signal. By applying Tayler series expansion for small $\alpha$ :

$$
y^{\prime}=A e^{j \omega t}(1+j \alpha \sin (\Omega t)
$$

expanding using sine identity gives:

$$
y^{\prime}=A e^{j \omega t}\left(1+\frac{\alpha}{2}\right)\left(e^{j \Omega t}-e^{-j \Omega t}\right)=A\left(e^{j \omega t}+\frac{\alpha}{2}\left(e^{j(\omega+\Omega) t}-e^{j(\omega-\Omega) t}\right)\right) .
$$

Identity in Bessel function yields:

$$
A e^{j \omega t+j \alpha \sin (\Omega t)}=A e^{j \omega t}\left[J_{0}(\alpha)+\sum_{k=1}^{\infty} J_{k}(\alpha) e^{j k \Omega t}+\sum_{k=1}^{\infty}(-1)^{k} J_{k}(\alpha) e^{-j k \Omega t}\right] .
$$

For intensity modulation system we can use equation 5.11 as simplified below:

$$
y^{\prime}=A e^{j \omega t}\left(1+\frac{\alpha}{2}\right)\left(e^{j \Omega t}-e^{-j \Omega t}\right)=A e^{j \omega t+j \alpha s i n(\Omega t)}=A\left(e^{j \omega t}\right)+\frac{A \alpha}{2 j}\left(e^{j(\omega+\Omega) t}-e^{j(\omega-\Omega) t}\right)
$$

or simply,

$$
y^{\prime}=A\left(e^{j \omega t}\right)+\frac{A \alpha}{2 j}\left(e^{j(\omega+\Omega) t}-e^{j(\omega-\Omega) t}\right) .
$$

For our single leg input case by which single electrode MZM is used to modulate a signal consisting of of two service signals, consider the following:

$$
u(t)=\alpha \sin \left(\Omega_{1} t\right)+\beta \sin \left(\Omega_{2} t\right)
$$

Again, the Mach Zender modulation will result in a phase shift that is proportional to the applied modulating electric signal as follows:

$$
y^{\prime}=M(y(t), u(t))=A e^{j \omega t+j\left(\alpha \sin \left(\Omega_{1} t\right)+\beta \sin \left(\Omega_{2} t\right)\right)} .
$$

This can be rewritten as:

$$
y^{\prime}=M(y(t), u(t))=A e^{j \omega t}\left[e^{j \alpha \sin \left(\Omega_{1} t\right)}\right]\left[e^{j \beta \sin \left(\Omega_{2} t\right)}\right] .
$$


The phase shift is proportional to the applied voltage as well as the refractive index change per volt for the MZM according to the equation:

$$
\Delta \Phi_{i}(t)=\frac{\pi}{V_{\pi}} u_{i}(t)
$$

$V_{\pi}$ is the termed as the full wave voltage which is equivalent to the voltage level that can cause the maximum phase shift of $\pi$. Now, let us define a new phase shift coefficient as:

$$
\eta=\frac{\pi}{V_{\pi}} .
$$

Here, we observe that both the signal voltage and $\eta$ are resposible for the phase shift. Bessel function identity applied on Equation 5.17 gives:

$$
\begin{array}{r}
y^{\prime}=E_{\text {OUT }}(t)=A e^{j \omega t}\left[J_{0}(\eta \alpha)+\sum_{k=1}^{\infty} J_{k}(\eta \alpha) e^{J_{k} \Omega_{1} t}+\sum_{k=1}^{\infty}(-1)^{k} J_{k}(\eta \alpha) e^{-j k \eta \Omega_{1} t}\right] \\
{\left[J_{0}(\eta \beta)+\sum_{k=1}^{\infty} J_{k}(\eta \beta) e^{J_{k} \Omega_{2} t}+\sum_{k=1}^{\infty}(-1)^{k} J_{k}(\eta \beta) e^{-j k \Omega_{2} t}\right]}
\end{array}
$$

Taking the first four sidebands only, we will have:

$$
y^{\prime}=E_{O U T, 4 S B}(t) \approx A e^{j \omega t}[B][C]
$$

where

$$
B=\left[J_{0}(\eta \alpha)+J_{1}(\eta \alpha) e^{j \Omega_{1} t}-J_{1}(\eta \alpha) e^{-j \Omega_{1} t}+J_{2}(\eta \alpha) e^{j 2 \Omega_{1} t}+J_{2}(\eta \alpha) e^{-j 2 \Omega_{1} t}\right]
$$

and

$$
C=\left[J_{0}(\eta \beta)+J_{1}(\eta \beta) e^{j \Omega_{2} t}-J_{1}(\eta \beta) e^{-j \Omega_{2} t}+J_{2}(\eta \beta) e^{j 2 \Omega_{2} t}+J_{2}(\eta \beta) e^{-j 2 \Omega_{2} t}\right]
$$

using sine and cosine identities

$$
\begin{array}{r}
y^{\prime}=E_{O U T, 4 S B}(t) \approx A e^{j \omega t}\left[J_{0}(\eta \alpha)+J_{1}(\eta \alpha) 2 j \sin \left(\Omega_{1} t\right)+J_{2}(\eta \alpha) 2 \cos \left(2 \Omega_{1} t\right)\right] \\
{\left[J_{0}(\eta \beta)+J_{2}(\eta \beta) 2 j \sin \left(\Omega_{2} t\right)+J_{1}(\eta \beta) 2 \cos \left(2 \Omega_{2} t\right)\right]}
\end{array}
$$


simplifying the product, rearranging terms, and disregarding non contributing frequency componants yield:

$$
\begin{aligned}
y^{\prime}=E_{\text {OUT,4SB }}(t) \approx A e^{j \omega t} & \left\{J_{0}(\eta \alpha) J_{0}(\eta \beta)+2 j\left[J_{1}(\eta \alpha) J_{0}(\eta \beta) \sin \left(\Omega_{1} t\right)+\right.\right. \\
& \left.J_{0}(\eta \alpha) J_{1}(\eta \beta) \sin \left(\Omega_{2} t\right)\right]+2\left[J_{0}(\eta \alpha) J_{2}(\eta \beta) \cos \left(2 \Omega_{1} t\right)+\right. \\
& \left.\left.J_{0}(\eta \beta) J_{2}(\eta \alpha) \cos \left(2 \Omega_{2} t\right)\right]\right\}
\end{aligned}
$$

substituting some variables to simplify gives:

$$
y^{\prime} \approx A e^{j \omega t}\left\{A B+2 j\left[C B \sin \left(\Omega_{1} t\right)+A D \sin \left(\Omega_{2} t\right)\right]+2\left[A F \cos \left(2 \Omega_{1} t\right)+B E \cos \left(2 \Omega_{2} t\right)\right]\right\}
$$

where,

$A=J_{0}(\eta \alpha) B=J_{0}(\eta \beta) C=J_{1}(\eta \alpha)$

$D=J_{1}(\eta \beta) E=J_{2}(\eta \alpha) F=J_{0}(\eta \beta)$.

\subsubsection{Derivation of Modulation Equation for Two Signal Model}

The following section describes the step by step development of the mathematical models for two signal input (one leg model) modulation. For simplicity we first consider two signals that have identical frequencies and then we will expand the discusion for the general case.

Considering the two electrical input signals:

$$
u_{1}(t)=\alpha \sin \left(\Omega_{1} t\right)
$$

and

$$
u_{2}(t)=\beta \sin \left(\Omega_{2} t\right)
$$

are applied separately to the two legs of MZM and

$$
y(t)=A e^{j \omega t}
$$

is the optical carrier signal from countinuous light wave source with amplitude of $A$ and optical frequency $\omega$. The two branch Mach Zender modulation causes phase shift 


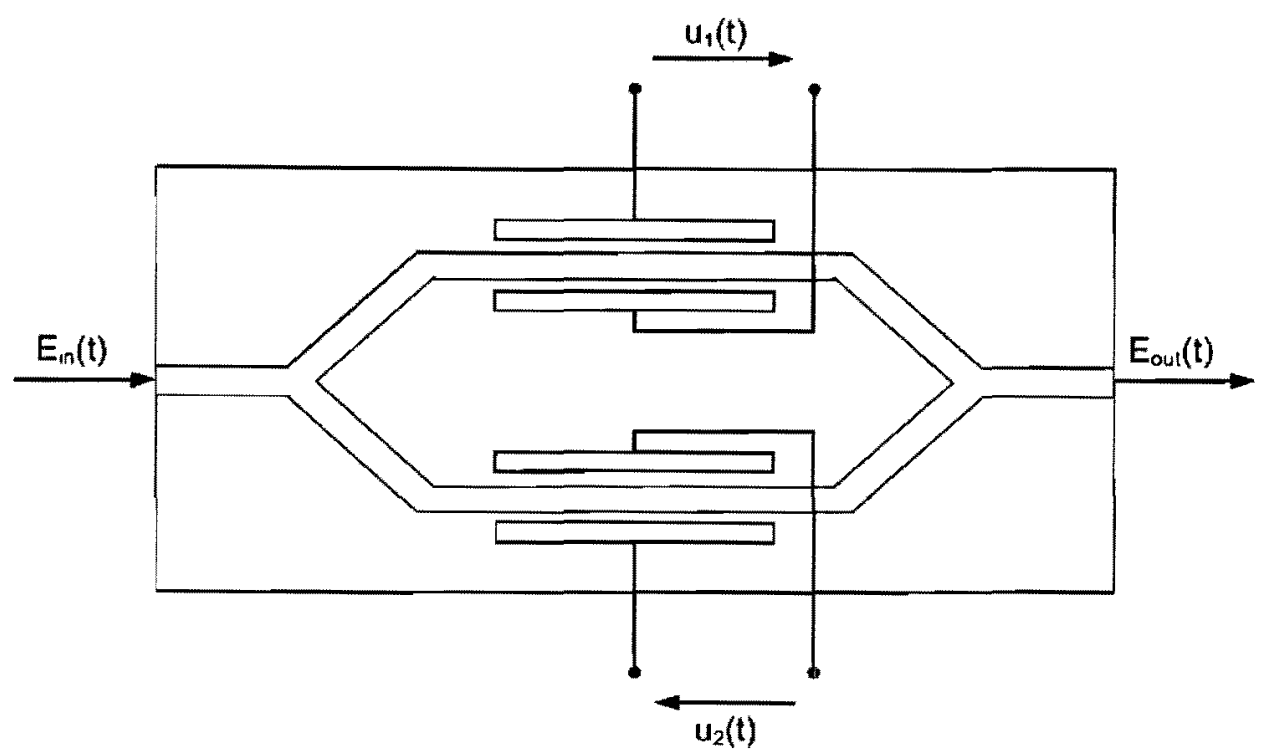

Figure 5.2: A physical structure representation of the two signal input $Y$ branch MZM.

to the optical signals that reach at the other end of the $\mathrm{Y}$ branch MZM in proportion to each of the applied sinusoidal modulating electric signals as follows:

$$
y^{\prime}=M\left(y(t), u_{1}(t), u_{2}(t)\right)=A e^{j \omega t+j \alpha \sin \left(\Omega_{1} t\right)}+A e^{j \omega t+j \beta \sin \left(\Omega_{2} t\right)} .
$$

Now, let us consider the electric field of the modulated optical signal that comes out of the MZM modulator. $\Delta \Phi_{1}(t)$ and $\Delta \Phi_{2}(t)$ are the phase changes at the two arms of the MZM due to the opto electric effect (Pockel's effect). From earlier discusions the change in phase is given by the relation:

$$
\Delta \Phi_{i}(t)=\frac{\pi}{V_{\pi}} u_{i}(t)
$$

where $u_{i}=1,2$ represents the voltage at each leg of the MZM. First, let us consider the electric field of the modulated optical signal at MZM output :

$$
y^{\prime}=E_{\text {out }}(t)=A e^{j \omega t}\left(e^{j \Delta \Phi_{1}(t)}+e^{j \Delta \Phi_{2}(t)}\right) .
$$


Moreover, if we assume that the MZM is biased at quadrature point then the bias voltage will satisfy the sequence:

$$
V_{b}=\frac{(2 n-1)}{2} V_{\pi}
$$

where $n=1,2,3, \ldots$, must be applied between the arms. If we apply $V_{b}$ at the arm 1 of the MZM, sinusoidal signals with amplitude $\alpha$ and $\beta$ and modulaing electrical signal frequency $\Omega_{1}$ and $\Omega_{2}$ at the arms 1 and 2 , respectively, then equation 5.18 will give us:

$$
\left\{\begin{array}{l}
\Delta \Phi_{1}(t)=\eta \alpha \sin \left(\Omega_{1}(t)\right)-\frac{\pi}{2} \\
\Delta \Phi_{2}(t)=\eta \beta \sin \left(\Omega_{2}(t)\right) .
\end{array}\right.
$$

Rewriting Equation 5.29 using the Euler's formula given by Equation 5.31 and substituting the relations above into it will lead to a Bessel function series of Equation 5.32 below:

$$
\begin{aligned}
& y(t)=A e^{j \omega t}\left(e^{\Delta \phi_{i}(t)}\right)=A e^{j \omega t}\left[\operatorname { c o s } \left(\Delta \phi_{i}(t)+j \sin \left(\Delta \phi_{i}(t)\right], i=1,2 ;\right.\right. \\
& y^{\prime}=E_{O U T}(t)=\left[\left(J_{0}(\eta \beta)-j J_{0}(\eta \alpha)\right)\right. \\
&+\sum_{(k=1)}^{\infty}\left(j(2 k-1)(\eta \alpha)+j J_{(2 k-1)}(\eta \beta)\right) \sin \left((2 k-1) \Omega_{1}(t)\right) \\
&\left.+\sum_{k=1}^{\infty}\left(j_{2 k}(\eta \beta)-j J_{2 k}(\eta \alpha)\right) \cos \left(2 k \Omega_{2}(t)\right)\right] A e^{j \omega t} .
\end{aligned}
$$

Therefore, the electric field at the MZM output given by Equation 5.29 can be expressed as a sum of Bessel functions series shown here. In this case $J_{n}(x)$ represents the Bessel function of order $\mathrm{n}$. With this consideration, the first two side-bands correspond to the lower and upper bands of a double side-band(DSB) signal. For simplicity, choosing only four side bands and assuming the two signals have same the frequency, now Equation 5.32 is possibly approximated for mathematical analysis as follows [39]:

$$
y^{\prime}=E_{O U T, 4 S B}(t) \approx\left[E_{D C}+E_{k} \sin (\Omega t)+E_{2 k} \cos (2 \Omega t)\right] A e^{j \omega t}
$$


where

$$
\left\{\begin{array}{l}
E_{D C}=J_{0}(\eta \beta)-j J_{0}(\eta \alpha) \\
E_{k}=2 J_{1}(\eta \alpha)+j 2 J_{1}(\eta \beta) \\
E_{2 k}=2 J_{2}(\eta \beta)-j 2 J_{2}(\eta \alpha) .
\end{array}\right.
$$

For general case, in which the two electrode MZM input (two leg model) consisting of two distinctive service signals, consider the following: $u_{1}(t)=\alpha \sin \left(\Omega_{1} t\right)$ and $u_{2}(t)=\beta \sin \left(\Omega_{2} t\right)$ are destinctive signals. The Mach Zender modulation will result in the phase shift proportional to the applied modulating electric signals as follows:

$$
y^{\prime}=M\left(y(t), u_{1}(t), u_{2}(t)\right)=A e^{j \omega t+j \alpha \sin \left(\Omega_{1} t\right)}+A e^{j \omega t+j \beta \sin \left(\Omega_{2} t\right)} .
$$

This can be rewritten as:

$$
y^{\prime}=M\left(y(t), u_{1}(t), u_{2}(t)\right)=A e^{j \omega t}\left\{\left[e^{j \alpha \sin \left(\Omega_{1} t\right)}\right]+\left[e^{j \beta \sin \left(\Omega_{2} t\right)}\right]\right\}
$$

By using the Bessel function identity for each part:

$$
\begin{array}{r}
y^{\prime}=E_{\text {OUT }}(t)=A e^{j \omega t}\left\{\left[J_{0}(\eta \alpha)+\sum_{k=1}^{\infty} J_{k}(\eta \alpha) e^{j k \Omega_{1} t}+\sum_{k=1}^{\infty}(-1)^{k} J_{k}(\eta \alpha) e^{-j k \Omega_{1} t}\right]+\right. \\
\left.\left[J_{0}(\eta \beta)+\sum_{k=1}^{\infty} J_{k}(\eta \beta) e^{j k \Omega_{2} t}+\sum_{k=1}^{\infty}(-1)^{k} J_{k}(\eta \beta) e^{-j k \Omega_{2} t}\right]\right\} .
\end{array}
$$

Taking the first four sidebands only, we will have:

$$
y^{\prime}=E_{O U T, 4 S B}(t) \approx A e^{j \omega t}\{[B]+[C]\}
$$

where

$$
B=\left[J_{0}(\eta \alpha)+J_{1}(\eta \alpha) e^{j \Omega_{1} t}-J_{1}(\eta \alpha) e^{-j \Omega_{1} t}+J_{2}(\eta \alpha) e^{j 2 \Omega_{1} t}+J_{2}(\eta \alpha) e^{-j 2 \Omega_{1} t}\right]
$$

and

$$
C=\left[J_{0}(\eta \beta)+J_{1}(\eta \beta) e^{j \Omega_{2} t}-J_{1}(\eta \beta) e^{-j \Omega_{2} t}+J_{2}(\eta \beta) e^{j 2 \Omega_{2} t}+J_{2}(\eta \beta) e^{-j 2 \Omega_{2} t}\right] .
$$


Here, it is important to compare this result with the result of Equation 5.21 and notice the difference. Now, using sine and cosine identities

$$
\begin{aligned}
y^{\prime}=E_{O U T, 4 S B}(t) \approx A e^{j \omega t}\{ & {\left[J_{0}(\eta \alpha)+J_{1}(\eta \alpha) 2 j \sin \left(\Omega_{1} t\right)+J_{2}(\eta \alpha) 2 \cos \left(2 \Omega_{1} t\right)\right]+} \\
& {\left.\left[J_{0}(\eta \beta)+J_{2}(\eta \beta) 2 j \sin \left(2 \Omega_{2} t\right)+J_{1}(\eta \beta) 2 \cos \left(2 \Omega_{2} t\right)\right]\right\} . }
\end{aligned}
$$

Substituting parameters for simplicity provides:

$$
\left.y^{\prime} \approx A e^{j \omega t}\left\{A+j 2 C \sin \left(\Omega_{1} t\right)+2 E \cos \left(2 \Omega_{1} t\right)\right]+B+j 2 F \sin \left(2 \Omega_{2} t\right)+D 2 \cos \left(2 \Omega_{2} t\right)\right\}
$$

where

$$
\begin{aligned}
& A=J_{0}(\eta \alpha) B=J_{0}(\eta \beta) C=J_{1}(\eta \alpha) \\
& D=J_{1}(\eta \beta) E=J_{2}(\eta \alpha) \quad F=J_{0}(\eta \beta) .
\end{aligned}
$$

\subsection{Channel Model}

In the transmission channel the signal is subjected to several losses and distortion. In this project it is tried to analyze and incorporate several channel properties of optical domain signal in the simulation. Attenuation is important as it is a determining factor for the distance between successive point to signal restoration. The optical fiber won over its competitor copper lines when its attenuation becomes lower than $5 \mathrm{~dB} / \mathrm{km}$, however today's general purpose optical fibers has an attinuation as low as $0.2 \mathrm{~dB} / \mathrm{Km}$. The attenuation of the signal is linear with the length of the transmission optical fiber length with the attenuation coefficient $\alpha$. For instance, with optical fiber length of 25 $\mathrm{km}$ and $\alpha 0.2 \mathrm{~dB} / \mathrm{km}$, attenuation will be $25 \times 0.2=5 \mathrm{~dB}$.

The other important factor in the transmission channel is the dispersion which is the characteristics of the carrier frequencies. This causes the broadening of the carrier light wave as it travels along the channel. As the signal disperses each pulse broaden and may overlap with the neighboring pulses and cause a phenomenon known as inter symbol interference (ISI). In order to avoided the overlap, the bit rate in the channel 
must be maintained with in the limit by which the wave disperse to its maximum. The following relation defines the relationship between maximum dispersion and bit rate limit (BT):

$$
B_{T} \leq \frac{1}{(2 \tau)}
$$

with the carrier light wave frequency $\omega$ and signal frequency of $\Omega$ the frequency domain dispersion of the signal can be given as:

$$
H(\Omega)=\alpha e^{j(\omega-\Omega)^{2}}
$$

where, $\omega \gg \Omega$. Since the two signal frequencies are close to each other (as compared to the carrier light wave frequency), the effect of the channel characterstics is assumed to be identical for both and therefore there is no need to develop a separate transmission model for the two cases under consideration.

\subsection{Detection Model}

The detection of optical signal and extracting the intelligible electrical message at the destination of the network is one of the most important stage in the optical fiber communication system. Selecting the right optical detecting devices and designing efficient detection model is crucial in the overall performance of the systems. The detecting elements extract and interpret the information contained and transported by the optical signal. The main purpose of the detecting devices is to convert the optical power in the optical fiber to alternating electrical voltage or current. Due to losses and distortion in the transmission channel, the signal is weak and distorted when it arrives at the receiver. Therefore, the detecting device must meet certain requirements to effectively detect this weak and distorted signal. The major requirements are sensitivity at particular transmission wavelength, minimum additional noise and fast response. Moreover detectors should also satisfy additional requirements such as insensitivity for temperature change, compatible physical dimension, cost and long life depending on the type of applications they are called for. In order to understand the operation of detector it is important to define some parameters which characterizer the detectors. One of the basic parameter for detectors is quantum efficiency $(\eta)$ and defined as follows. The 
quantum efficiency $(\eta)$ is the fraction of incident photons which are absorbed by the photo detector and generate electrons which are collected at the detector terminal [10]:

$$
\eta=\frac{\text { number of electrons collected }}{\text { number of incident photons }}
$$

Hence,

$$
\eta=\frac{r_{e}}{r_{p}}
$$

where, $r_{p}$ is the incident photon rate(photon per second) and $r_{e}$ is the corresponding electron rate(electron per second). Since quantum efficiency is defined in terms of number of particles involved, additional parameter is required which involve the energy and power contained to give the measurement a more physical meaning. With this regard, responsivity $\mathbb{R}$ is defined which can better characterize the performance of the photo detecting device. Responsivity is defined as the ratio of the output electric current to the incident optical power:

$$
\mathbb{R}=\frac{I_{P}}{P_{O}}
$$

By considering the energy of a photon to be $E=h f$, where (h, Plank's constant), the incoming photon rate can be given as:

$$
r_{P}=\frac{P_{O}}{h f}
$$

The electron rate therefore is:

$$
r_{e}=\eta P_{O}=\frac{\eta P_{O}}{h f}
$$

From the rate of electron we can get the output photocurrent as:

$$
I_{P}=\frac{\eta P_{O} e}{h f}
$$

where, $e$ is the charge of electron. From Equation 5.42 an 5.45 we can define the responsivity as:

$$
\mathbb{R}=\frac{\eta e}{h f}
$$


The power received at a point $\mathrm{x}$ in the optical fiber is described by equation:

$$
p(x)=P_{O}\left(1-e^{-\alpha_{s}(\lambda) x}\right)
$$

where, $\alpha_{s}$ is the absorption coefficient at wavelength $\lambda$, and $P_{0}$ is the power at the original source [16]. The current therefore can be written as:

$$
I_{P}=\frac{e}{h c} P_{O}\left(1-e^{-\alpha_{s}(\omega)}\right)(1-\mathbb{R} c)
$$

considering the reflexivity at the entrance, here $\mathrm{c}$ is the speed of light.

\subsubsection{Photo Detector Noise}

A thorough understanding of the source, characteristics and interrelationship of the the various noise in a photo detector is very important in order to design an efficient fiber optical communication system receivers. Generally there are several noise sources persistant in the fiber optics communication networks. These can be categorized as man-made noise (e.g., neighboring transformers, motors, and television stations), noise from natural source (such as., lightning, earthquakes, cosmic sources), or noise from the intrinsic characteristics of to the optical detector. For this study we are most interested on the later noise source described, namely noise from optical detectors. Generally the sensitivity of the photo detector device lie on the noise caused by the randomness of the fluctuating current and voltage at terminal of the device output that caused by the incident optical signal. This is noise caused primarily due to the statistical nature of the conversion process. There is also thermal noise associated to the signal processing and amplification of the electrical signal. In most cases, it is the noise current that determines the minimum optical power level that can be detected. Sensitivity is usually referred to as the minimum detectable optical power. It is the optical power necessary to produce a photocurrent of the same magnitude as the root mean square (rms) of the noise current, or equivalent to a signal-to-noise ratio of one [16]. The power signal-to nose ratio $\mathrm{S} / \mathrm{N}$ at the output of an optical receiver is defined as :

$$
\frac{S}{N}=\frac{\text { signal power from photocurrent }}{\text { photodetector noise power }+ \text { amplifier noise power }}
$$


Considering the intrinsic characteristics of the optical detector, quantum noise and dark current noise are the major kinds of the noises in the optical detector. However, the analysis of the receiver circuitry shall assume the presence of thermal noise associated with photo detector load resistance in the amplifier circuitry. In the following equations the mean square value of quantum noise, bulk dark current, surface dark noise and thermal noise are given [16]:

$$
\begin{aligned}
i_{Q}^{2} & =2 e I_{p} B M^{2} F(M) \\
i_{D B} 2 & =2 e I_{D} B M^{2} F(M) \\
i_{D S}^{2} & =2 e I_{L} B \\
i_{T}^{2} & =\frac{4 k_{B} T}{R_{L}} .
\end{aligned}
$$

In the formula $e$ is charge of electron, $B$ is bandwidth, $M$ is the multiplication factor for internal amplification of APD and $F(M)$ is a function associated with $M$. The total noise in the optical receiver circuit therefore is given as:

$$
N=2 q I_{p} B M^{2} F(M)+2 q I_{D} B M^{2} F(M)+2 q I_{L} B+\frac{4 k_{B} T}{R_{L}} .
$$

Now equation 5.49 yields:

$$
\frac{S}{N}=\frac{i_{p}^{2} M^{2}}{2 e I_{p}} \frac{M^{2} F(M)+2 e I_{D} B M^{2} F(M)+2 e I_{L} B+\frac{4 k_{B} T}{R_{L}}}{.} .
$$

In order to simplify the effort of mathematical calculation, considering the PIN diode as detector yields $M=1$. Moreover if we assume the major contribution of the noise comes from the quantum noise source, then thermal noise, the bulk and surface dark current noise can be neglected for our case. Therefore, Equation 5.55 can be written as:

$$
\frac{S}{N}=\frac{i_{P}{ }^{2}}{2 e I_{p} B}
$$

Better understanding of the nature and type of the noise, helps to closely estimate the behavior of the detection model. It is also important to select the kind of detector which has a better sensitivity and noise parameter based upon the type of signal we are dealing. Generally the output current at the end of the detector signal is defined 
by the equation:

$$
I=\mathbb{R} P(t)+N(t)
$$

where, $I$ represents the output current, $\mathbb{R}$ is the responivity of the detector element $\mathrm{P}(\mathrm{t})$ is the optical power received at time $t$ and $N(t)$ is the total noise at the time of detection of particular signal. The noise at the detection is estimated using the equation 5.57 and equation 5.54 as to model and simulate the receiver module of the network.

\subsection{Bit Error Rate Determination}

One of the most common performance evaluation parameters in digital communication system is Bit Error Rate (BER). It could be defined as the ratio of erroneous bits received to the total received bit in a given period of time. There are several ways of measuring BER for digital signal. The most common way is to tally the number of errors in a given interval of time and to divide it with the total number of bit transmitted in that interval. Such method needs a means to record the entire received signal and have the means to know the transmitted signal and compare the two. Since the transmitted signal has gone through the channel and is subjected to noise, we don't have a means to know what is the correct signal transmitted while we are at the receiver side. However, this problem is usually solved by means of sending a known set of data to the receiver (pilot signal) so that the receiver has a pre-knowledge of the sent data.

In digital systems BER could be evaluated using the simulated equivalent of a system using computer and digital means. This doesn't exactly replicate the real system and it just provides a means to approximate the behavior of the actual system. The other means to determine the BER is by analytical and mathematical means. This is done by using the probability distribution function of the SNR in order to determine the probability of error. This is the most adopted method by many literature and researchers. For our discussion, we will consider this method. Basically we consider the BER in order to compare the performance of the two models we are considering in this project. In digital communication noise is usually assumed to have Gaussian distribution. This noise distribution is implemented to have the estimate of SNR to provide a certain amount of BER. In order to derive the error distribution therefore we 
first consider the Guassian distribution given below:

$$
p(s)=\frac{1}{\sigma \sqrt{2 \pi}} \exp \left\{-\left[(s-m)^{2} / 2 \sigma^{2}\right]\right\}
$$

where $m$ is the mean of the distribution $\sigma$ is the standard deviation and s represents the signal strength.

In the digital signal transmission the expected values at the receiver are a signal level of either 0 or 1 . Whenever there is error the zeros must have been changed to 1 or the one must have been changed to 0 . Let us assume there is a threshold value of voltage above which all the received voltages are assumed to be one and all the voltage levels below are considered to be 0 . Therefore we can define the probability of receiving 1 if the signal sent was zero, $p(1 / 0)$, similarly the probability of receiving zero if the signal sent was $1, p(0 / 1)$. We define the probability of sending 1 and 0 as $p(1)$ and $p(0)$, respectively. Then, the total probability of error bits is given as :

$$
p(e)=p(1) p(0 / 1)+p(0) p(1 / 0)
$$

Assuming the data transmitted is not biased, the probability of occurrence of 0 and 1 are equal and yields $\mathrm{p}(0)=\mathrm{p}(1)=0.5$. Now defining the threshold voltage as $V_{d}$, if a binary 1 sent and the noise current is negative and make the difference $V_{s}+V_{N}$ less than $V_{d}$, the received signal becomes binary 0 and error will occur. Similarly, if a binary 0 sent and the noise current is positive and $V_{s}+V_{N}$ sums up to greater than $V_{d}$, the received signal becomes binary 1 and error will occur. Assuming the $i_{\text {sig }}$ is the peak current of the signal when the transmission is binary 1 and no signal is sent when the binary data is 0 , and mean square noise current in both state is identical and equal to $\overline{i_{N}{ }^{2}}$. Hence we can rewrite Equation 5.58 as follows for both cases:

$$
\begin{gathered}
p_{1}(i)=\frac{1}{\overline{i_{N}{ }^{2}} \frac{1}{2} \sqrt{2 \pi}} \exp \left\{-\left[\left(i-i_{s i g}\right)^{2} / 2 \overline{i_{N}^{2}}\right]\right\}, \\
p_{0}(i)=\frac{1}{\overline{i_{N}{ }^{2}} \sqrt{\frac{1}{2}} \sqrt{2 \pi}} \exp \left\{-\left[(i-0)^{2} / 2 \overline{i_{N}{ }^{2}}\right]\right\}
\end{gathered}
$$

Since the integral of such function is hard to evaluate, the customary practice is to rewrite the error function and it's complementary, in order to estimate the value of the 
probability distribution function. Therefore we can write the probabilities using these erf and erfc as:

$$
p(0 / 1)=\frac{1}{2}\left[1-\operatorname{erf} \frac{\left|i_{s i g}-i_{D}\right|}{\overline{i_{N}{ }^{\frac{1}{2}}} \sqrt{2}}\right]=\frac{1}{2}\left[\operatorname{erfc} \frac{\left|i_{\text {sig }}-i_{D}\right|}{\frac{i_{N}{ }^{2}}{\frac{1}{2}} \sqrt{2}}\right]
$$

Similarly

$$
p(1 / 0)=\frac{1}{2}\left[1-\operatorname{erf} \frac{\left|0-i_{D}\right|}{{\overline{i_{N}}}^{\frac{1}{2}} \sqrt{2}}\right]=\frac{1}{2}\left[\operatorname{erf} c \frac{\left|0-i_{D}\right|}{i_{N}{ }^{\frac{1}{2}} \sqrt{2}}\right]
$$

with the assumption discussed previously where the number of 0 's and 1's transmitted or received are equal, $\mathrm{p}(0)=\mathrm{p}(1)=1 / 2$, Therefore we can rewrite Equation 5.59 as:

$$
p(e)=\frac{1}{2}[p(0 / 1)+p(1 / 0)]
$$

Substituting the results in equation 5.62 and 5.63 gives:

$$
p(e)=\frac{1}{2}\left\{\left[\frac{1}{2}\left[\operatorname{erfc} \frac{\left|i_{s i g}-i_{D}\right|}{{\overline{i_{N}}}^{\frac{1}{2}} \sqrt{2}}\right]+\frac{1}{2}\left[\operatorname{erf} c \frac{\left|0-i_{D}\right|}{{\overline{i_{N}}}^{\frac{1}{2}} \sqrt{2}}\right]\right\} .\right.
$$

If we let the $V_{D}$ to be at the midpoint between the signal values of bit 1 and bit 0 , which corresponds to minimum probability of errors for bits in most electrical systems, we can simplify the above equation further as:

$$
p(e)=\frac{1}{2}\left\{\left[\frac{1}{2}\left[\operatorname{erfc} \frac{\left|\frac{i_{s i g}}{2}\right|}{{\overline{i_{N}{ }^{2}}}^{\frac{1}{2}} \sqrt{2}}\right]+\frac{1}{2}\left[\operatorname{erfc} \frac{\left|-\frac{i_{\text {isi }}}{2}\right|}{{\overline{i_{N}}}^{\frac{1}{2}} \sqrt{2}}\right]\right\}\right.
$$

or simply:

$$
p(e)=\frac{1}{2}\left[\operatorname{erfc} \frac{i_{s i g}}{i_{N}{ }^{\frac{1}{2}} \sqrt{2}}\right] .
$$

From Equations 5.49 and 5.55 we have seen that the SNR of the signal at the detector could be written as the ratio of the peak signal power to the mean square noise power current. Therefore we can replace $\frac{S}{N}$ for $\frac{i_{s i g}^{2}}{i_{N}^{2}}$ and rewrite the above equation as:

$$
B E R=p(e)=\frac{1}{2} \operatorname{erfc}\left(\frac{\left(\frac{S}{N}\right)^{\frac{1}{2}}}{2 \sqrt{2}}\right) .
$$


Now we can use this relationship to plot our BER graphs.

\subsubsection{BER for One Leg Input Signal Model}

Using the above discussion on the derivation of the BER, now we will proceed in deriving the BER for the different models of this project. For amplitude modulation case, by considering Equation 5.14 which define the signal strength of single leg modulator and Equation 5.56 which define the SNR of the receiver and inserting this relationship into Equation 5.67 we can obtain BER versus SNR relationship for the system under study. Consider $i_{p}=A\left(e^{j \omega t}\right)+\frac{A \alpha}{2 j}\left(e^{j(\omega+\Omega) t}-e^{j(\omega-\Omega) t}\right)$, and $\frac{S}{N}=\frac{i_{P}{ }^{2}}{2 q I_{p} B}$. The estimate of the BER values for range of signal strength can be calculated for the current defined above. The the peak signal current is given as:

$$
i_{\text {sigmax }}=\left|A+\frac{-j A \alpha}{2}\right|
$$

for $\alpha=1$ we can estimate the maximum signal as:

$$
i_{\text {sigmax }}=\left|A+\frac{-j A}{2}\right|=1.118 A
$$

we can evaluate the BER of different SNRs using several values of $\alpha$ for electrical power and A for optical power strength.

However for the general case of modulation single signal input dervied at equation 5.23 we can determine the maximum signal by taking the estimated values from the coeffcients of the multiplying factor. Hence we can simplify the signal strength as:

$$
y^{\prime} \approx A\left\{J_{0}(\eta \alpha) J_{0}(\eta \beta)+2 J_{1}(\eta \alpha) J_{0}(\eta \beta)+2 J_{0}(\eta \alpha) J_{1}(\eta \beta)+2 J_{0}(\eta \alpha) J_{2}(\eta \beta)+2 J_{0}(\eta \beta) J_{2}(\eta \alpha)\right\}
$$

Using this estimation we can determine the BER for single signal input model. Different signal power levels could be taken to evaluate the SNR and the error function developed in the above section can be applied for BER plot. It is also useful to use computer programs to evaluate the Bessel function coefficients as well as the erfc function in order to plot the BER graph easily. In chapter 6 the respective BER plots are shown. 


\subsubsection{BER for Two Leg Input Signal Model}

Considering Equation 5.33 which define the signal strength of two leg modulator and Equation 5.56 which define the SNR of the receiver and inserting this relationship into Equation 5.67 we obtain the following values: The Bessel function coefficients are read from the table and inserted to determine the signal power strength as follows: $i_{P} \approx\left[E_{D C}+E_{k} \sin \left(\Omega_{1} t\right)+E_{2 k} \cos \left(2 \Omega_{2} t\right)\right] A e^{j \omega t}$, with:

$$
\left\{\begin{array}{l}
E_{D C}=(\eta \beta)-j J_{0}(\eta \alpha) \\
E_{k}=2 J_{1}(\eta \alpha)+j 2 J_{1}(\eta \beta) \\
E_{2 k}=2 J_{2}(\eta \beta)-j 2 J_{2}(\eta \alpha) .
\end{array}\right.
$$

As an example if we assume the voltage level for $\alpha=\beta=1 \mathrm{~V}$ and an MZM of biasing voltage $2 \mathrm{~V}$ is choosen; then $\eta=\pi / 2=1.57$. Therefore,

$$
\begin{aligned}
& \left.E_{D C}=J_{0}(1.57 \beta)-j J_{0}(1.57) \alpha\right)=|0.4752-j 0.4752| \\
& E_{k}=2 J_{1}(1.57 \alpha)+j 2 J_{1}(1.57 \beta)=|1.1334+j 1.1334| \\
& E_{2 k}=2 J_{2}(1.57 \beta)+j 2 J_{2}(1.57 \alpha)=|0.499-j 0.449| .
\end{aligned}
$$

The peak signal strength now could be estimated as

$$
i_{\text {sigmax }} \approx 2.9806 \mathrm{~A} \text {. }
$$

We can evaluate the BER for the model by using different signal power levels and evaluating the SNR and using the error function derived above. However, for the general case modulation of the two distinctive signal inputs dervied at Equation 5.37, we can determine the maximum signal strength by taking the estimated values from the coeffcients of the multiplying factor. Hence we can simplify the signal strength as:

$$
y_{\text {sigmax }}^{\prime} \approx A\left\{J_{0}(\eta \alpha)+2 J_{1}(\eta \alpha)+2 J_{2}(\eta \alpha)+J_{0}(\eta \beta)+J_{2}(\eta \beta)+2 J_{1}(\eta \beta)\right\} .
$$

Again, we can determine the BER for this model by using different signal power levels and evaluating the SNR and using the error function derived. By comparing this BER with the BER for single signal input, we can determine the performance differnce between the two models developed in this project. The comparison of the BERs for the two model is provided in results section of Chapter 6 . 


\section{Chapter 6}

\section{Simulation and Results}

\subsection{Simulation Setup}

In this section the setup of the simulation, analytical and simulation results and discussion have been presented. The simulation is organized first by formulating the mathematical model for modulation, transmission and the receiver/detector unit. The whole simulation process took place in MATLAB-Simulink software package. As indicated in Figure 4.1 and Figure 4.2 the complete system constitutes a signal flow of electrical domain to optical domain and then back to electrical domain pattern. Therefore, in order to identify the two different domain signals distinctively, as well as for the convenience of developing simulation software, the electrical domain signals are represented and simulated within the Simulink package while the optical domain signals are represented and processed in MATLAB development environment. Figure 6.1 shows the block diagram for the general flow of signals within the simulation environment. The simulation constitutes formulating pseudorandom digital words which represent the two services. The typical digital service signals and the subcarrier radio signals are simulated using the software package tools of the Simulink. The mixing, filtering and other electrical domain based processes are also incorporated within this facility. In the other hand the modulation of the electrical signal to light signal, the optical channel transmission and the optical detection model all are developed and simulated in MATLAB software development environment. The block diagrams at each stage clearly indicate each modular representation of the process involved and simulation in both development environments. The following flow routs indicate the direction of the 
signal path:

Electrical $\rightarrow$ Optical $\rightarrow$ Electrical

Simulink $\rightarrow$ Matlab $\rightarrow$ Simulink

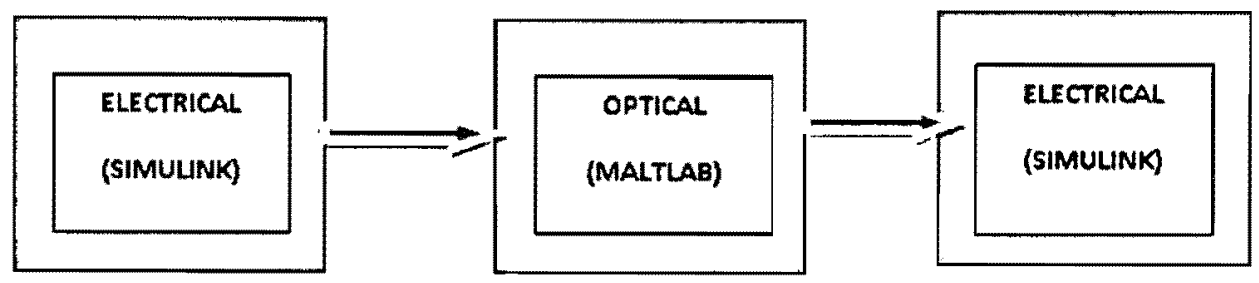

Figure 6.1: Block diagram of the signal flow for the simulation models.

As indicated in Figures 6.2,6.9 and 6.10, there are two kinds of simulation model developed in the Simulink. These models are termed as one leg and two leg model. The one leg model simulation is the implementation of the mathematical derivation of Chapter 5 Equations 5.9 to Equation 5.14 which consider a single signal input for the MZM. In this case, the two radio service signal are mixed together in the electrical domain before they are sent to modulate the optical signal. The other model which is specified as a two leg model is a representation of the two signals delivered separately to the MZM. These signals modulate the light wave separately and get mixed in the optical domain. The mathematical derivation of this model is shown in Chapter 5 Equation 5.28 to Equation 5.32 which assume the two signal delivered separately to MZM. As mentioned earlier the simulation of the electrical part is considered to be performed in the Simulink. The two subcarrier multiplexed signal are obtained by mixing the $1.25 \mathrm{~Gb} / \mathrm{s}$ signal with $5 \mathrm{GHz}$ sinusoidal signal and $622 \mathrm{Mb} / \mathrm{s}$ signal with $25 \mathrm{GHz}$ sinusoidal signal in Simulink modules. One of these radio signals is further mixed with band limited white noise with power $0.001 \mathrm{v}$ rms. The simulation architecture of the model, simulation results and discussion of each model is provided in the following section respectively. All the simulation devices and tools used in Simulink integrated software development environment are listed in Appendix A and their respective setup values are indicated. 


\subsection{One Leg Modulation Scheme}

In the one leg modulation scheme the two service signals are mixed in the electrical domain at Simulink phase. In the Simulink model, the simulation of radio signal is made using Bernoulli random digital simulator by setting the amplitude to 1 volt and the sampling period in such a way that the generated signal represents a $622 \mathrm{Mb} / \mathrm{s}$ random digital signal. The signal is mixed with a random white noise to simulate the noise persistence in the radio signals. The signal then mixed with $25 \mathrm{GHz}$ sinusoidal subcarrier signal to up convert the operating frequency of the radio range to microwave frequency range. Similarly, $1.25 \mathrm{~Gb} / \mathrm{s}$ signal is simulated using second Bernoulli digital word simulator and mixed with $5 \mathrm{GHz}$ sinusoidal subcarrier signal and the two signals are added together in the Simulink environment. The simulation time is carefully designed so that the simulation carries sufficient sample digital word from both the signal types. Since the simulation time for both signals take place simultaneously and exactly equal, care has to be taken to get sufficient samples from both types of signals. The length of simulation duration, simulation sample size and length of the available digital word pulses are indicated at respective simulation result figures.

The sample signal then fed to the MATLAB module which represents the optical part of the network. At several stages of the process, the signal is routed to time and frequency domain scopes to observe the characteristics at each stage and to compare with what is expected. Figure 6.2 shows the block diagram for different modular blocks organized in the Simulink tool set for one leg modulation scheme. In the MATLAB module of simulation which represents a super continuum light source produces a continuous light signal of wavelength $1550 \mathrm{~nm}$. In the MZM, the phase of the optical signal is shifted in accordance to the value of the modulating electrical signal strength which is driven from the Simulink stage. The mathematical derivations and equations developed in Chapter 5 are used to represent the MZM simulation. In the MATLAB programs some typical characteristics of MZM, such as the non-linearity effect has been modeled and included. 


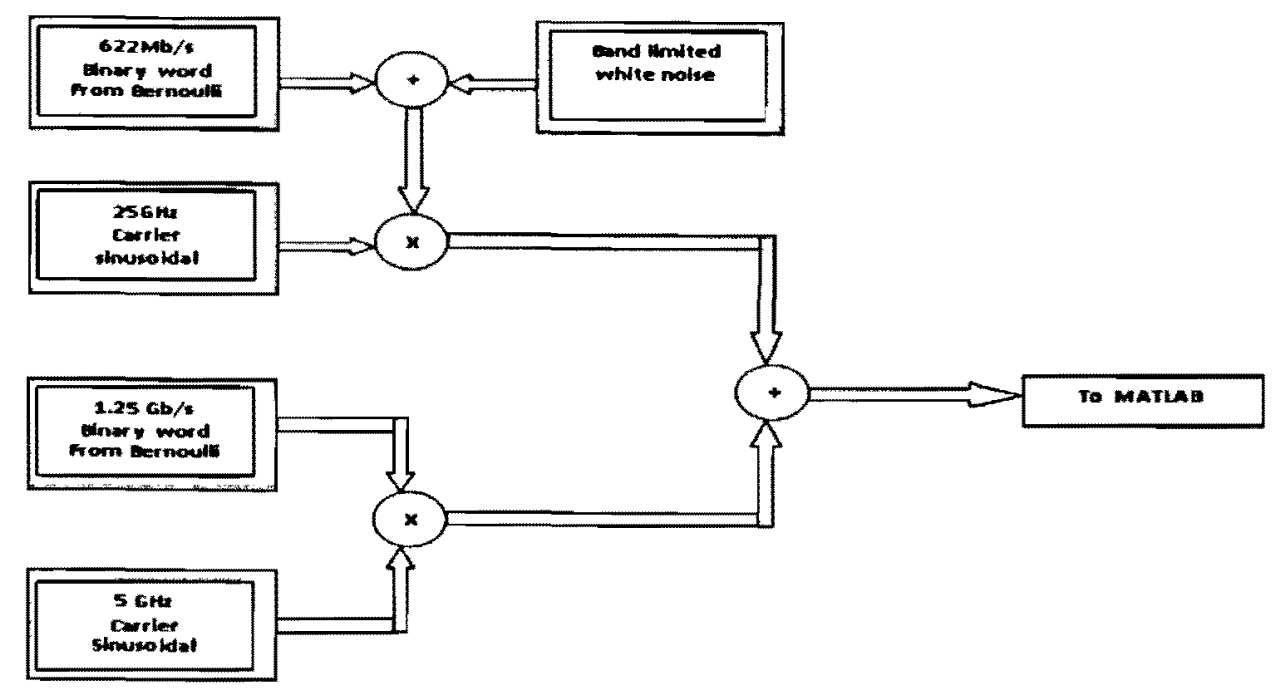

Figure 6.2: Block diagram of the flow of signal in Simulink phase of the simulation.

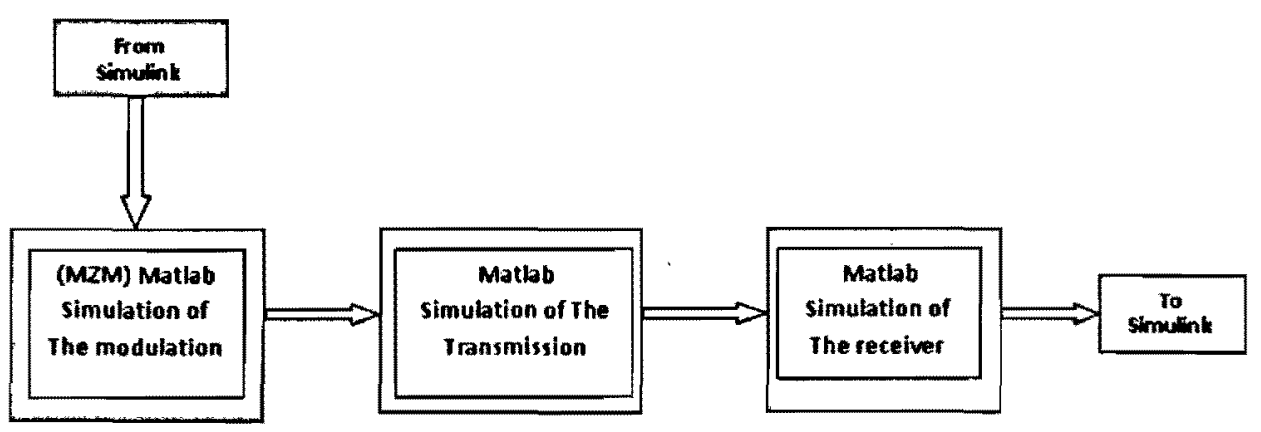

Figure 6.3: The block diagram representation for the signal flow in the MATLAB modules. 


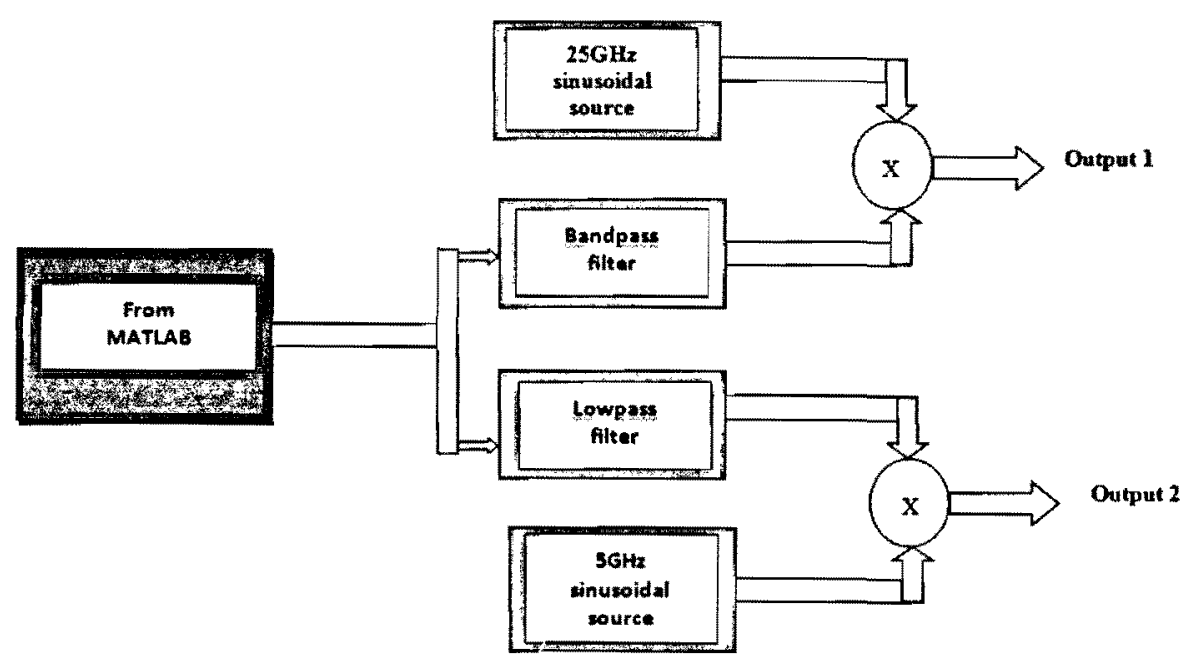

Figure 6.4: Block diagram for the Simulink stage following the MATLAB processes. The detected light signal now fed to different filter types and then the signals will mix to the original sinusoidal subcarrier in order to detect the transmitted message. 


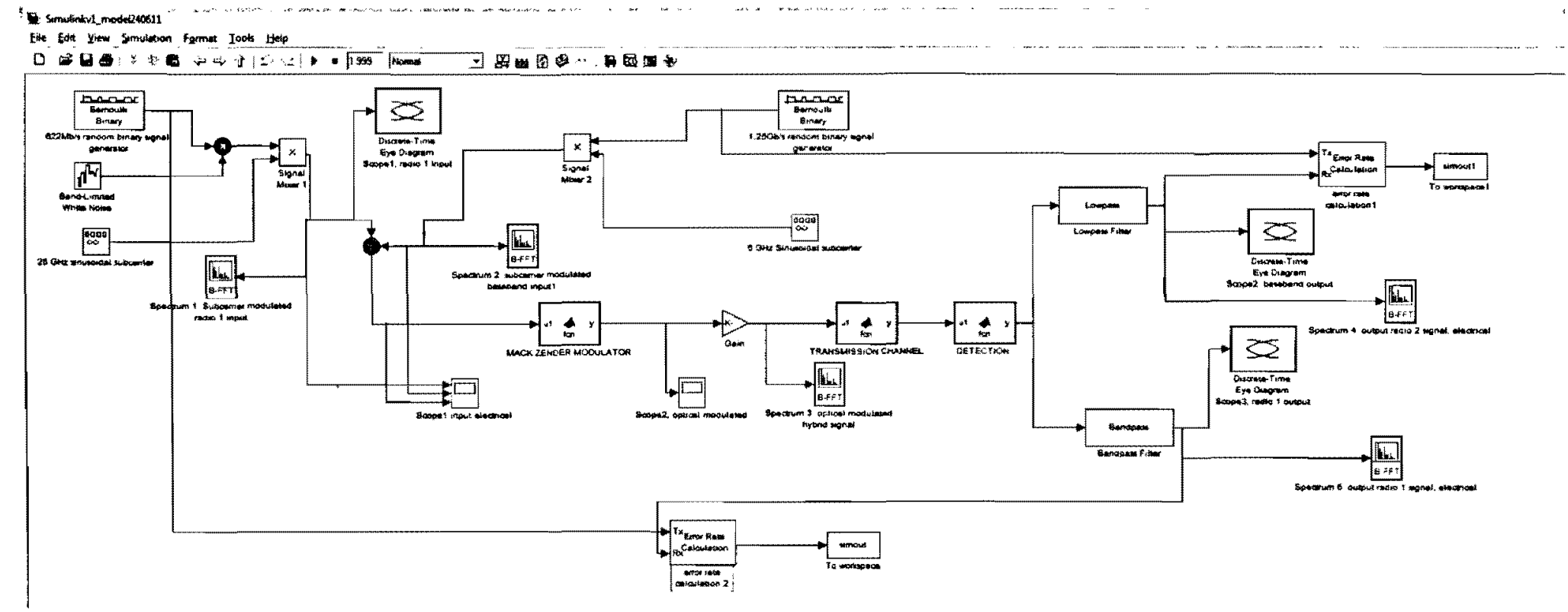

Figure 6.5: The schematic diagram representation for simulation of one leg input model. The Simulink and MATLAB modules with interconnection, measuring and display devices are shown. 
The two Bernoulli binary word generators as indicated in Figure 6.5 simulate two random binary sources with $622 \mathrm{Mb} / \mathrm{s}$ and $1.25 \mathrm{~Gb} / \mathrm{s}$ data rate. A random binary white noise which simulates noise characterstic is included. The spectral output is observed at several stages using the B-FFT spectrum analyzer. The two signals are first mixed with in the Simulink stage and they are routed to MATLAB package which simulates the optical stages of the process. Three MATLAB packages simulate the modulation, transmission and detection stages of the optical phase. Following the optical stage, the signals is fed back to Simulink for filtering, demodulation as well as error rate calculation and signal distortion measurements. The respective Simulink devices and their setup values are listed in Appendix A.

The first scope in Figure 6.6 indicates radio service 1 with $622 \mathrm{Mb} / \mathrm{s}$ source digital word and $25 \mathrm{GHz}$ subcarrier Sinusoidal signal. In the second scope, the $1.25 \mathrm{~Gb} / \mathrm{s}$ digital word with $5 \mathrm{GHz}$ subcarrier is shown. As indicated, 20 bits of word for $1.25 \mathrm{~Gb} / \mathrm{s}$ data rate signal require 16 nanosecond, and its $5 \mathrm{GHz}$ subcarrier requires 160 samples (0.1ns/sample). For the $622 \mathrm{Mb} / \mathrm{s}$ (with 1.6 nanosecond PW) signal about 10 bits of words could be simulated. The third scope shows the two signals added together in the Simulink stage. The mathematical model developed in Chapter 5 are used to simulate the MZM in MATLAB. Since the amplitude modulation (OOK) is considered, the signal in light domain is also zero when both the bits of the source are simultaneously zero. 


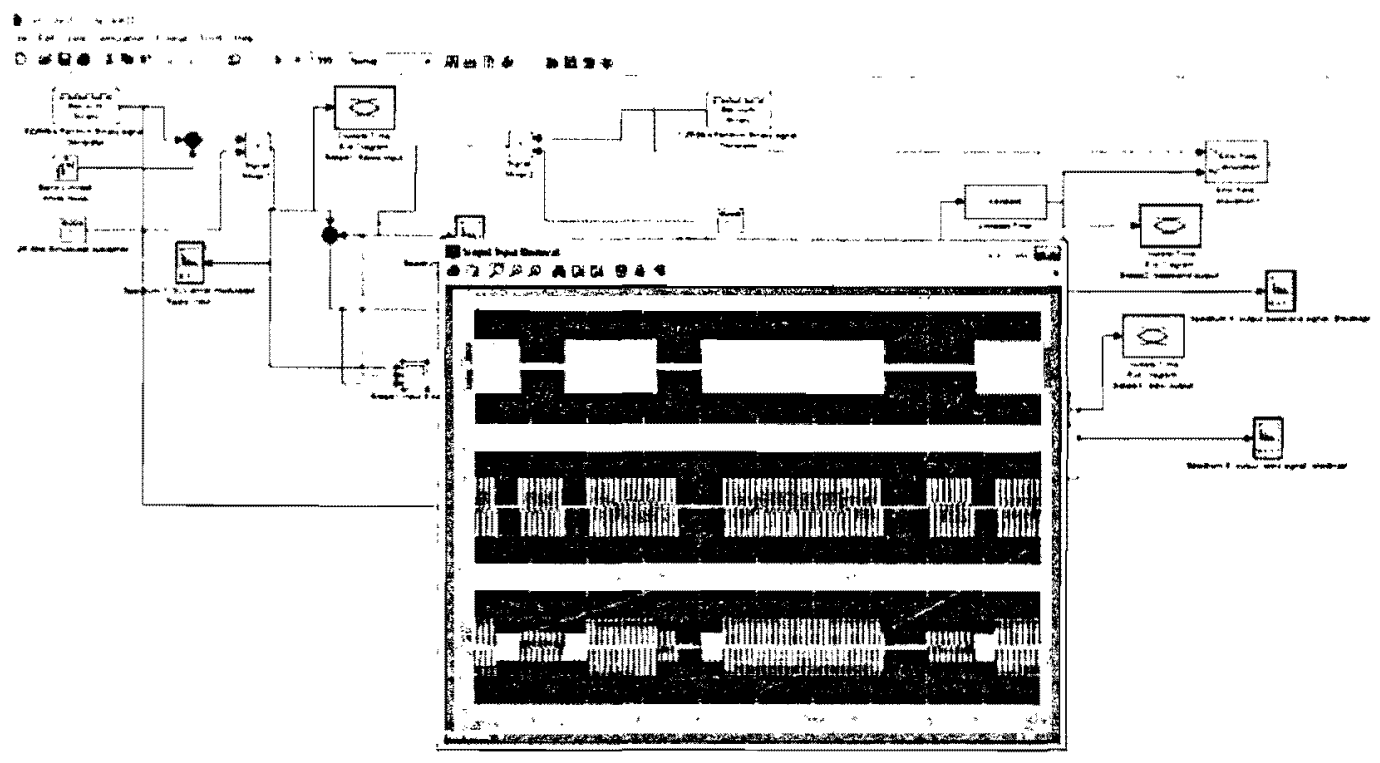

Figure 6.6: Time domain simulation display of the two input signals in Simulink stage.

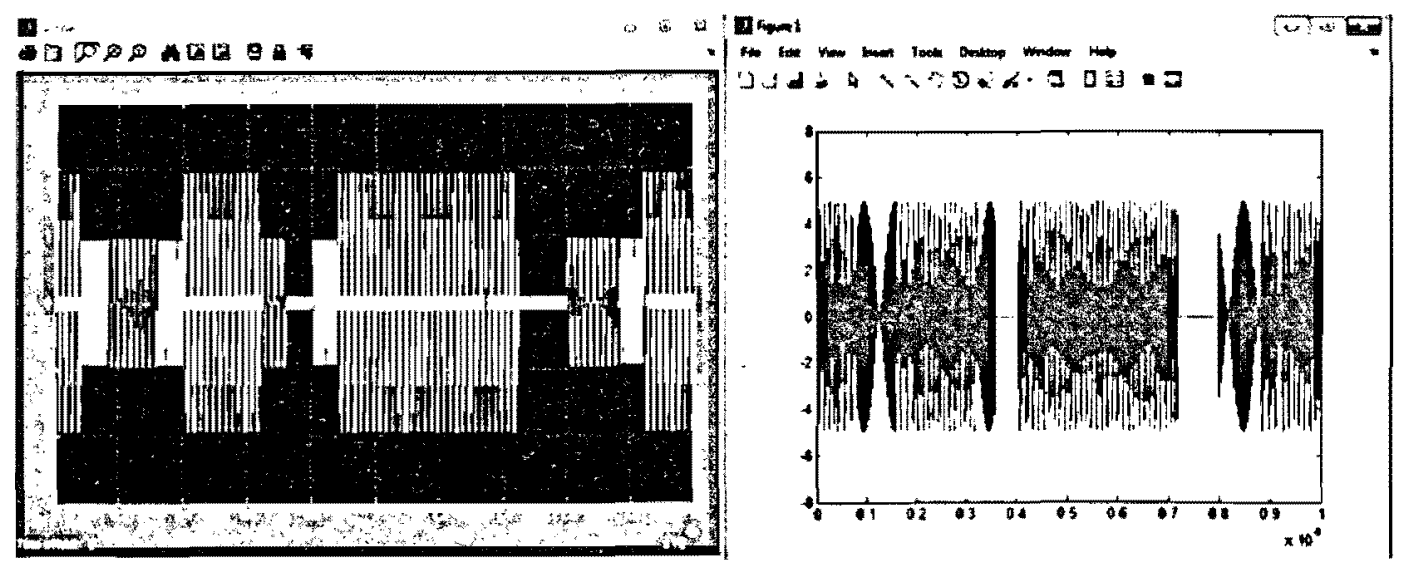

Figure 6.7: Time domain simulation comparison of the two interleaved signals in Simulink and MATLAB stages. 


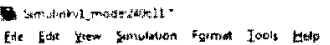

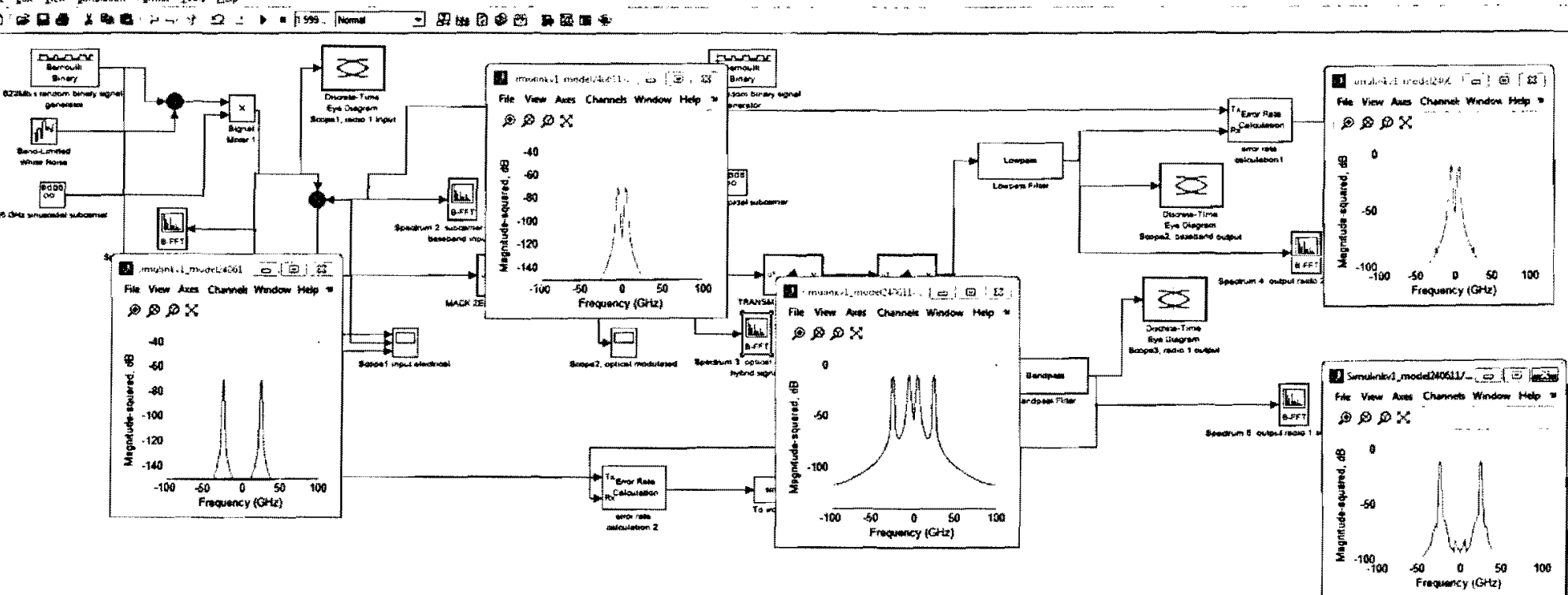

Figure 6.8: Frequency spectrum display for the simulation of single signal input model. 
In the spectrums display of Figure 6.8, red plots show the input/output spectrum of the double side band for radio service 1 with $622 \mathrm{Mb} / \mathrm{s}$ source digital word and $25 \mathrm{GHz}$ subcarrier sinusidial signal, while the green plots indicate the second service with $1.25 \mathrm{~Gb} / \mathrm{s}$ digital word and $5 \mathrm{GHz}$ subcarrier. Electrical power can be reduced at the simulation of the source so that the modulator can handle lower input power. The low power signal and power losses at the Simulink stage can be componsated by boosting up the power level for transmission range using amplifiers in the MATLAB stage. Insertion loss and attenuation can also be modeled in this stage. The blue plot in the middle shows the interleaved signals at the optical stage of the simulation.

\subsection{Two Leg Modulation Scheme}

In this model two radio simulation signal are both produced in the Simulink phase and both are fed to the MATLAB section separately. In the Simulink model, Bernoulli random binary word simulator is used. The first radio signal for simulation purpose is represented by setting the amplitude to 1 volt. The sampling period is set in such a way that it represents a $622 \mathrm{Mb} / \mathrm{s}$ random radio digital signal. The signal is mixed with a random noise to simulate the noise persistence in the radio signals. The signal then mixed with $25 \mathrm{GHz}$ sinusoidal signal to up convert the operating frequency of the radio range to higher frequency range. Similarly the second radio signal is generated using another Bernoulli digital word generator and mixed with $5 \mathrm{GHz}$ sinusoidal signal and the two signals are separately provided to the MATLAB block which simulates the MZM. The following block diagrams represent the flow of the signals in the simulation modules. The respective Simulink devices and their setup values are listed in Appendix A. 


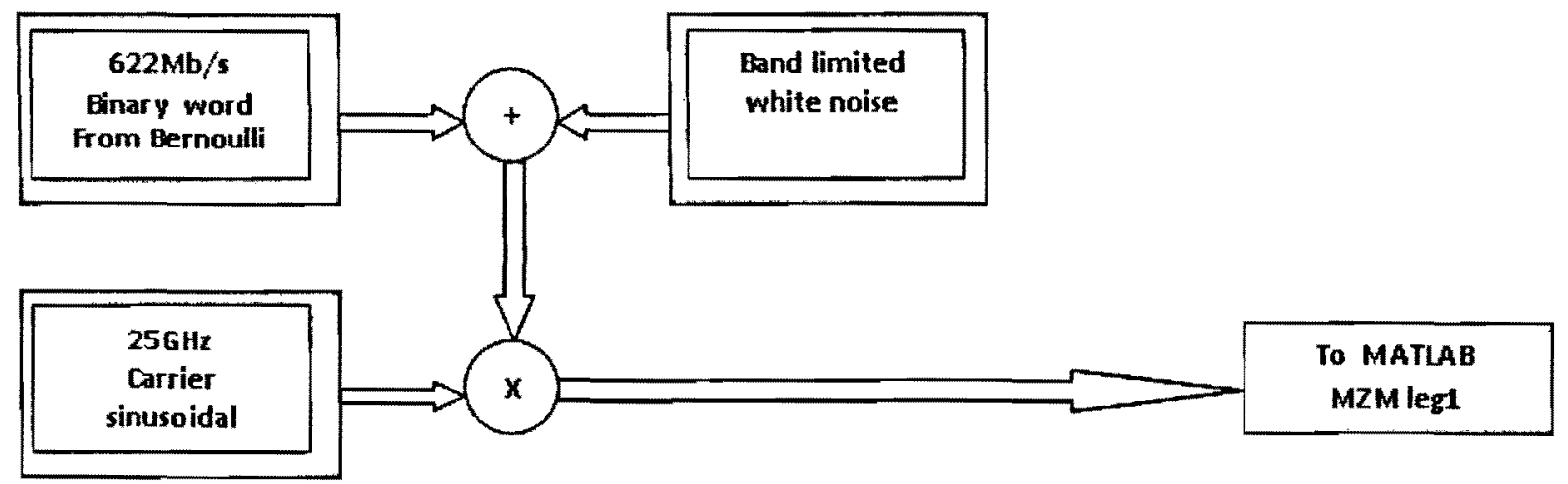

Figure 6.9: Block diagram for the simulation source for radio signal 1 in Simulink.

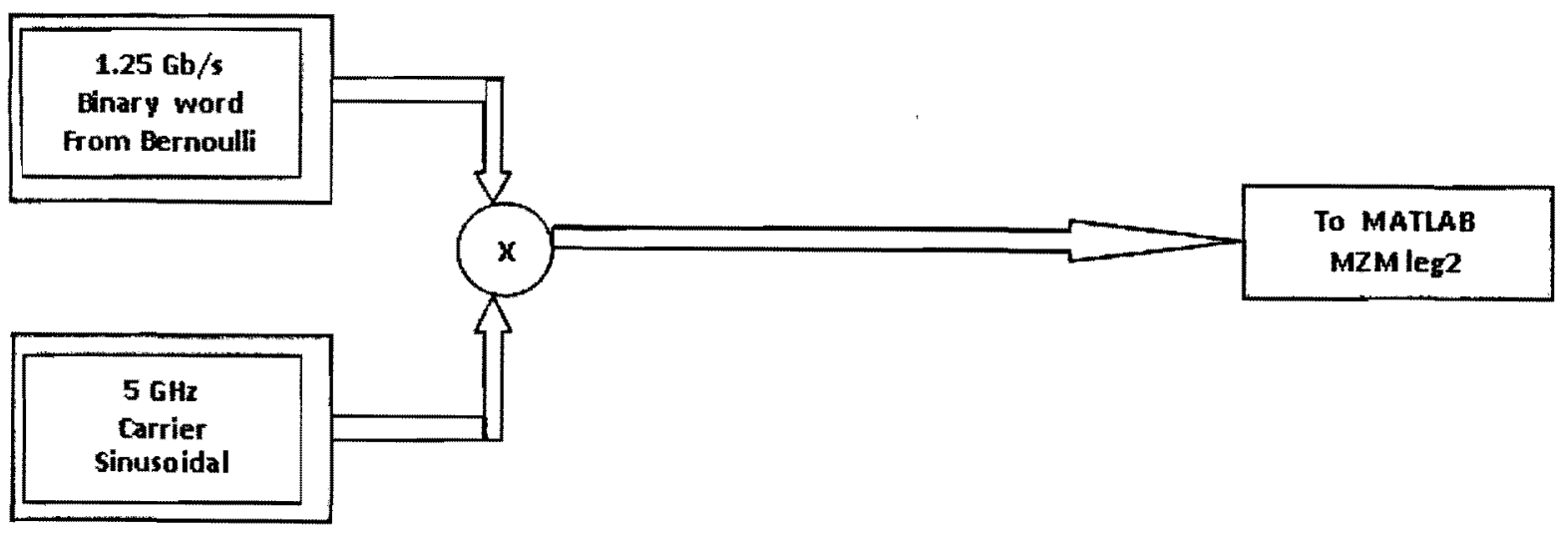

Figure 6.10: Block diagram for the simulation source for radio signal 2 in Simulink. 


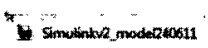

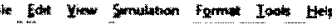

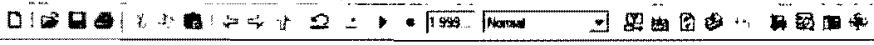

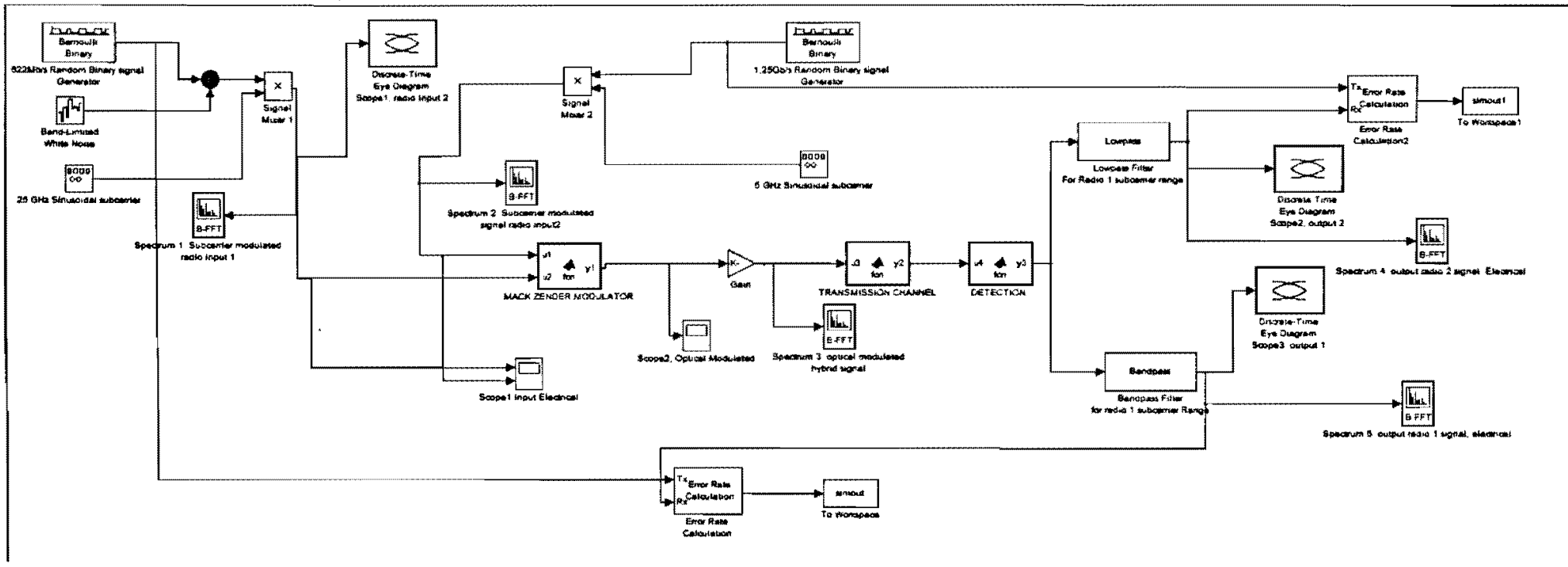

Figure 6.11: The schematic diagram representation for simulation of two leg input model. The Simulink and MATLAB modules with interconnection, measuring and display devices are shown. 
The two Bernoulli binary word generators indicated in Figure 6.11 simulate a random binary source with $622 \mathrm{Mb} / \mathrm{s}$ and $1.25 \mathrm{~Gb} / \mathrm{s}$ data rate. A random binary white noise which simulates noise characterstic is included. The spectral output is observed at several stages using the B-FFT spectrum analyzer. The two signals are separately sent to MATLAB module to get modulated with optical carrier. Three MATLAB packages simulate the modulation, transmission and detection stages of the optical phase. Following the optical stage the signals are fed back to Simulink for filtering, demodulation as well as error rate calculation and signal distortion measurements.

The first scope in Figure 6.12 indicates radio service 1 with $622 \mathrm{Mb} / \mathrm{s}$ source digital word and $25 \mathrm{GHz}$ subcarrier sinusidial signal. In the second scope, the $1.25 \mathrm{~Gb} / \mathrm{s}$ digital word and $5 \mathrm{GHz}$ subcarrier is shown. As indicated in the window, 20 bits of word for $1.25 \mathrm{~Gb} / \mathrm{s}$ require $16 \mathrm{~ns}$, its $5 \mathrm{GHz}$ subcarrier requires 160 samples $(0.1 \mathrm{~ns} / \mathrm{sample})$. Considering the same length of simulation time for the $622 \mathrm{Mb} / \mathrm{s}$ (with $1.6 \mathrm{~ns}$ pulse width), about 10 bits of words could be simulated.

The two signals are separately routed and modulated in the MATLAB section of the model. Since the mathematical equation representation of the MZM is phase modulation based, the hybrid optical signal will not zero out when both the signals are simultaneously zero. This is different from the amplitude model we observed in the single signal input case. 


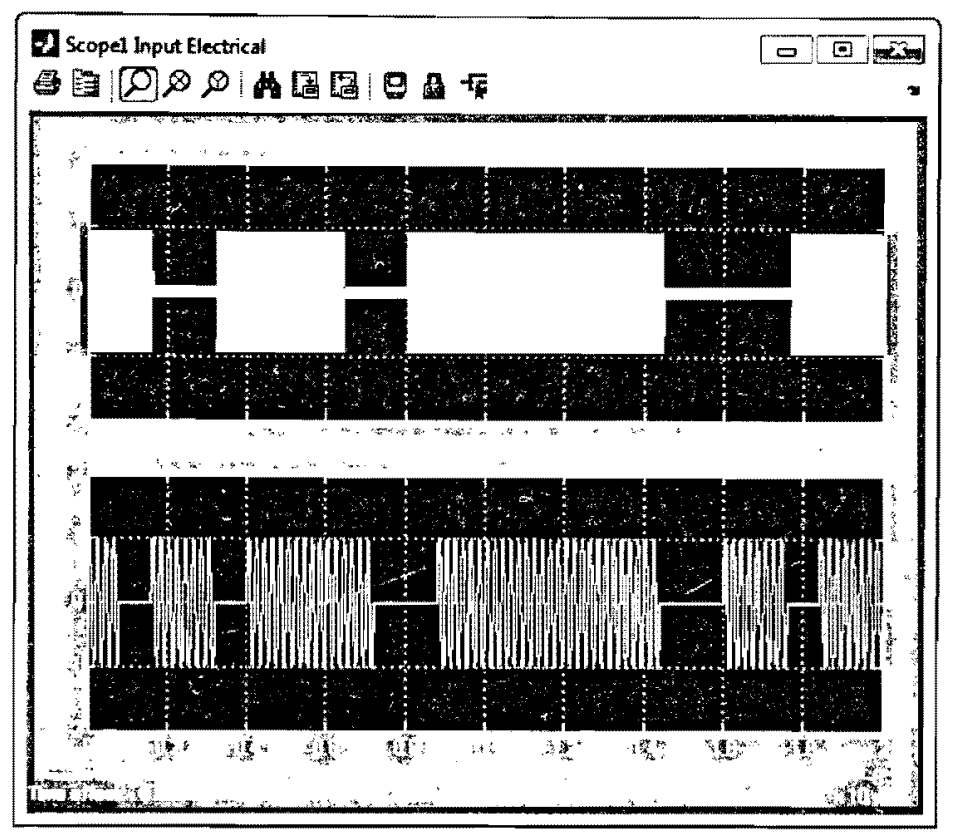

Figure 6.12: Time domain simulation display of the two input signals in Simulink stages.

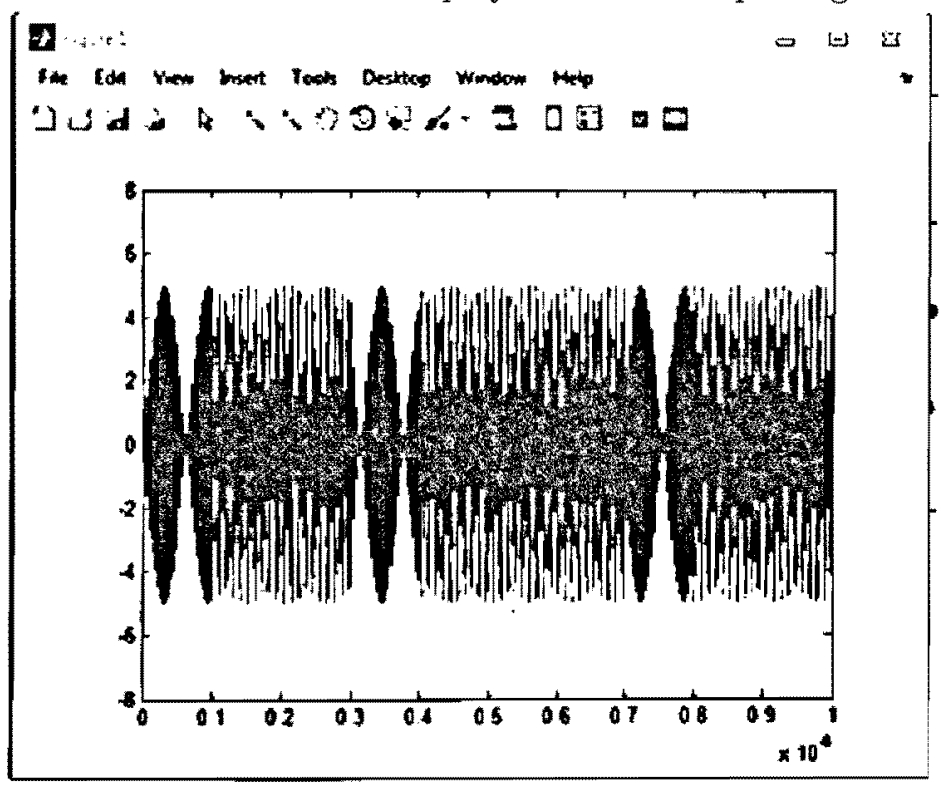

Figure 6.13: Time domain simulation display of the two input signals after optical carrier modulation in MATLAB simulation. 


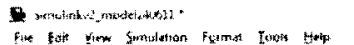
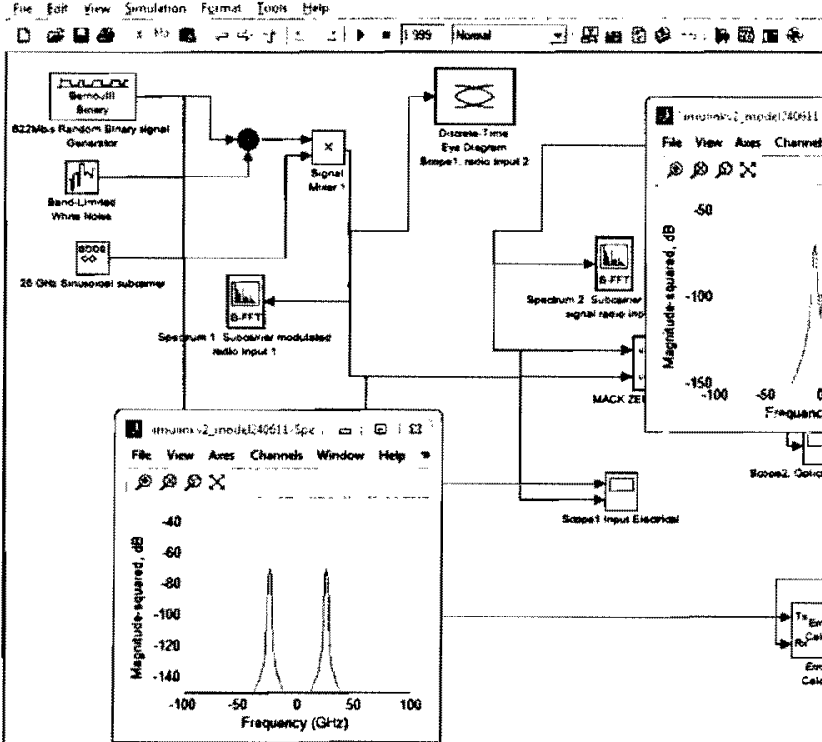

D

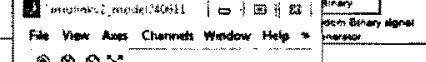

$\rightarrow \longrightarrow$

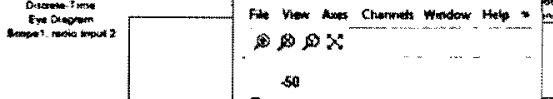

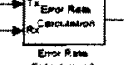
$00 x$

g.
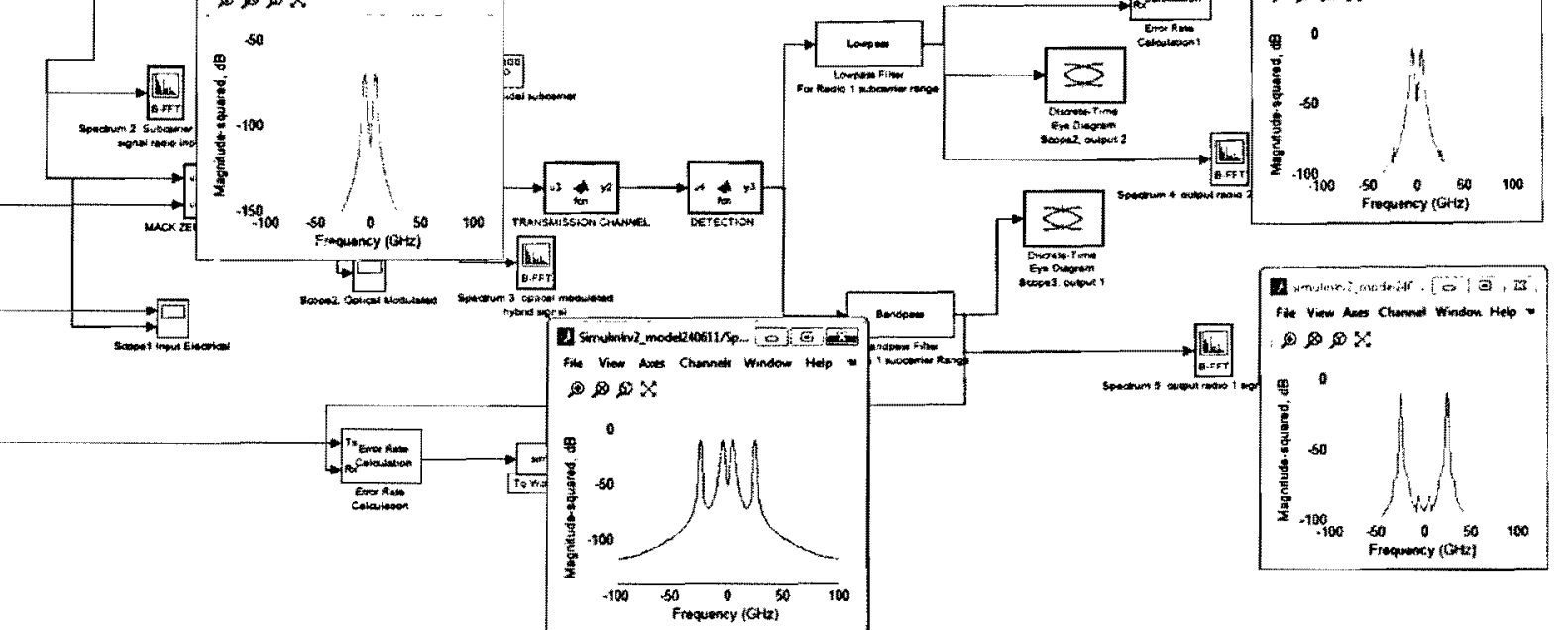

8

Figure 6.14: Frequency spectrum display for the simulation of two signal input model. 
In the graph shown in Figure 6.14, red plots show the input/output spectrum of the double side band for radio service 1 with $622 \mathrm{Mb} / \mathrm{s}$ source digital word and $25 \mathrm{GHz}$ subcarrier sinusidial signal, while the green plots indicate the second service with $1.25 \mathrm{~Gb} / \mathrm{s}$ digital word and $5 \mathrm{GHz}$ subcarrier for the two leg signal simulation case. Electrical power can be reduced at the simulation of the source so that the modulator can handle lower input power. The low power signal and power losses at the Simulink stage can be componsated by boosting up the power level for transmission range using amplifiers in the MATLAB stage. Insertion loss and attenuation can also be modeled in this stage. The blue plot in the middle shows the interleaved signals at the optical stage of the simulation.

\subsection{BER Plots}

The BER plot is the most common performance evalution tool for digital signal transmission systems. Following the discusion in Chapter 5 the BERs of the two models have been ploted and presented in this section. Several assumption are mode to simplify the set up of the plots. At each steps, the assumption and discusion is provided. consider the Equation 5.24 derived in Chapter 5:

$$
y^{\prime} \approx A e^{j \omega t}\left\{A B+2 j\left[C B \sin \left(\Omega_{1} t\right)+A D \sin \left(\Omega_{2} t\right)\right]+2\left[A F \cos \left(2 \Omega_{1} t\right)+B E \cos \left(2 \Omega_{2} t\right)\right]\right\}
$$

where,

$A=J_{0}(\eta \alpha) B=J_{0}(\eta \beta) C=J_{1}(\eta \alpha)$

$D=J_{1}(\eta \beta) E=J_{2}(\eta \alpha) F=J_{0}(\eta \beta)$.

In order to determine the maximum signal strength let us approximate the magnitude of the coefficients by:

$$
M_{O N E L E G}=\sqrt{(A B)^{2}+(2 C B)^{2}+(2 A D)^{2}+(2 A F)^{2}+(2 B E)^{2}} .
$$

We use this value in order to evaluate the SNR and program the BER plot for the one leg input model. With the same discusion, the two leg input model magnitude of the 
Bessel function parameters can be approximated by:

$$
M_{T W O L E G}=\sqrt{A^{2}+(2 C)^{2}+(2 E)^{2}+B^{2}+(2 F)^{2}+(2 D)^{2}} .
$$

The BER curve can be ploted using two approaches. The first approach is by considering fixed parameters of the optical domain including the launching optical power strength. The power of the two electrical signal sources are varied in a specified pattern. The SNR values for various electrical domain input power can be evaluated and BER versus input electrical power is plotted. This approach involves determining the Bessel function coefficients at each stage and evaluating the maximum signal strength in electrical domain using the equations above. The second approach is by keeping the electrical domain power at a fixed level and observe the variation in SNR and BER for changes in the strength of the launching optical power. Both approaches are programmed for some selected parameters and the following result is observed and presented.The two approaches doesn't give identical result however they can be used to compare the performance of the two models under consideration.

In Figure 6.15, both input signals are kept at equal strength and varied uniformly.

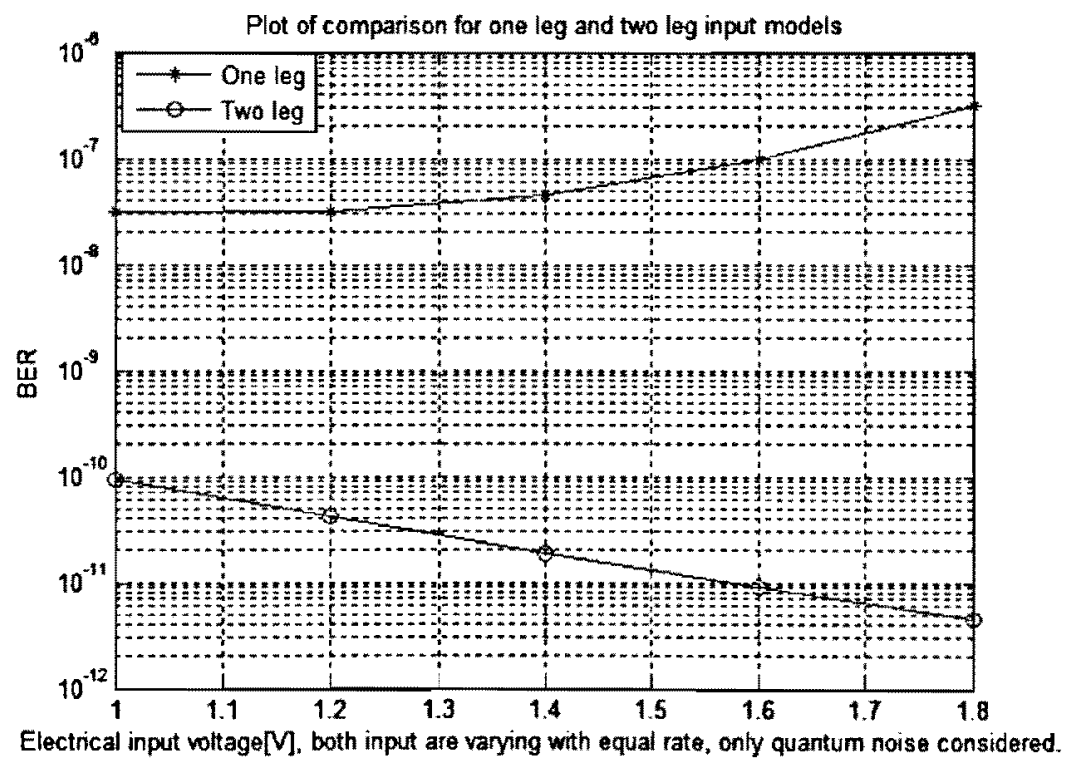

Figure 6.15: BER plot for performance comparison of the two models with the quatum noise assumption.

The values of the varying voltages are shown on the plot. The BER is determined by 


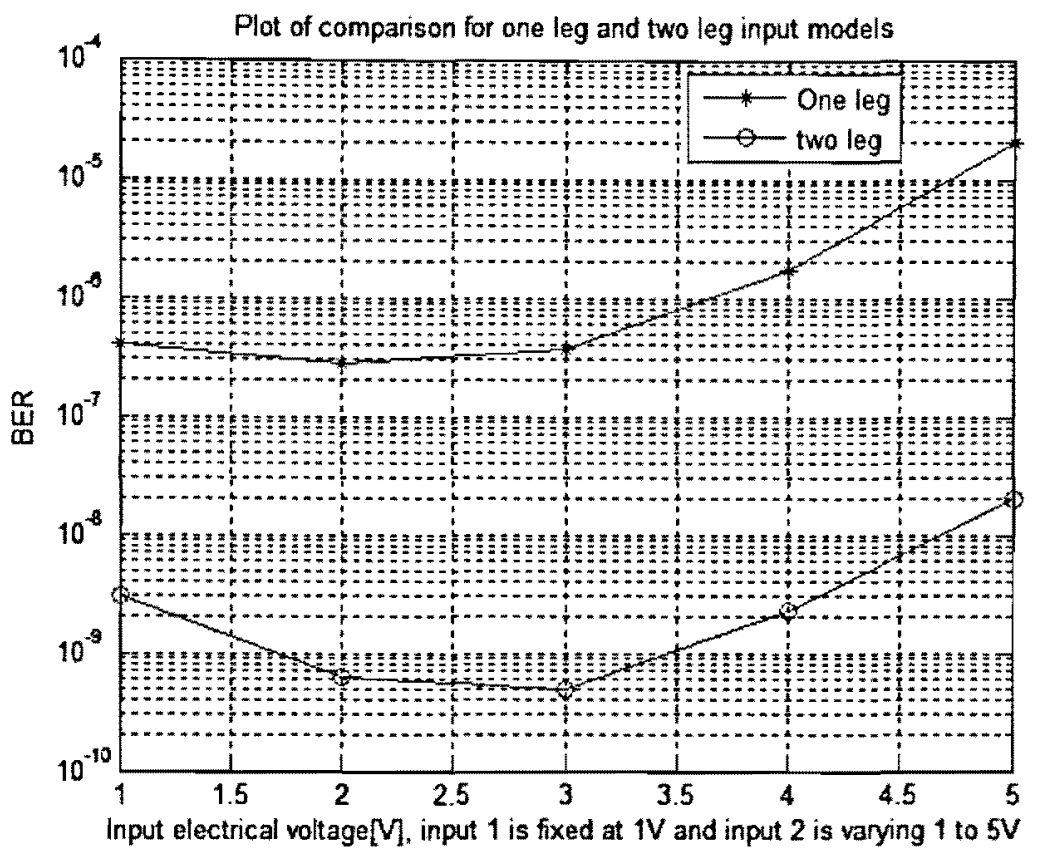

Figure 6.16: BER plot for comparison of the two models. One of the input signal is fixed ( $\alpha$ in this case) and $\beta$ is varying from low to high values as shown in the graph.

sending $0.115 \mu \mathrm{A}$ optical signal. In this case the full wave voltage is kept at $2 \mathrm{~V}$. The responsivity of the detecor is assumed .90 . For this particular measurment bandwidth is taken as $6 \mathrm{GHz}$. The quantum noise is assumed to be the major contributer for the total noise at the receiver, therefore all other noise souces are assumed to be zero including the thermal noise. As can be observed in the graph, the two leg model is remarkabely better in performance for all the values at which the measurments are taken.

The BER plot of Figure 6.16 is determined by sending fixed $0.115 \mu \mathrm{A}$ optical signal power. In this case the full wave voltage is kept at $2 \mathrm{~V}$. The responsivity of the detecor is considerd to be 0.90 . The quantum noise is assumed to be the major contributer for the total noise at the receiver, therefore all other noises, including the thermal noise, are assumed to be zero. As can be observed in the graph, the two leg model is remarkabely better for all the values at which the measurments are taken.

In Figure 6.17, both the input signals are kept at equal strength and varied uniformly. The values of the varying voltages are shown on the plot. The BER is deter- 


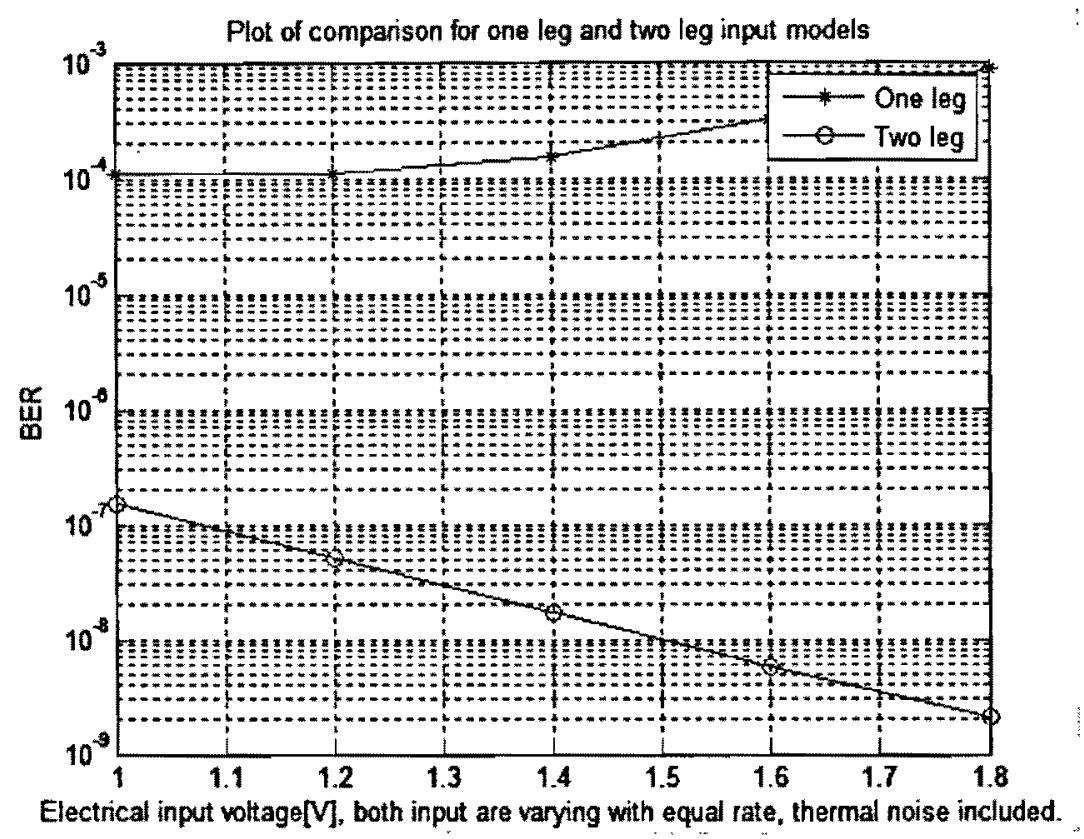

Figure 6.17: BER plot for performance comparison of the two models with $\alpha$ and $\beta$ varying simultaneously and thermal noise assumed to be the major contributer.

mined by sending fixed $0.115 \mu \mathrm{A}$ optical signal power. In this case the full wave voltage is kept at $2 \mathrm{~V}$. The responsivity of the detecor is taken to be 0.90 . For this particular measurment bandwidth is taken as $6 \mathrm{GHz}$. The thermal noise is included in this measurment. To make it remarkable, thermal noise is taken 10 fold of the quantum noise. As can be observed in the graph that the two leg model is remarkabely better for all the values at which the measurments are taken. The BER has shift upward (as compared to the no thermal noise plot) for both models as expected from the incorporation of significant amount of thermal noise.

For Figure 6.18 the values of the launching optical power are indicated in current unit. The BER plot is determined by sending $0.095 \mu \mathrm{A}$ to $0.0115 \mu \mathrm{A}$ optical signal. The half wave voltage is kept at $2 \mathrm{~V}$ and the amplitude of the electrical power is kept at $1 \mathrm{~V}$ level. The responsivity of the detecor is considerd as 0.90 . For this particular measurment bandwidth is taken as $10 \mathrm{GHz}$. The quantum noise is assumed to be the major contributer for the total noise at the receiver, therefore all other noises, includingthe thermal noise, are assumed to be zero. The difference in the BER curves increases as the transmission power increases. As can be observed in the graph, the two leg model 


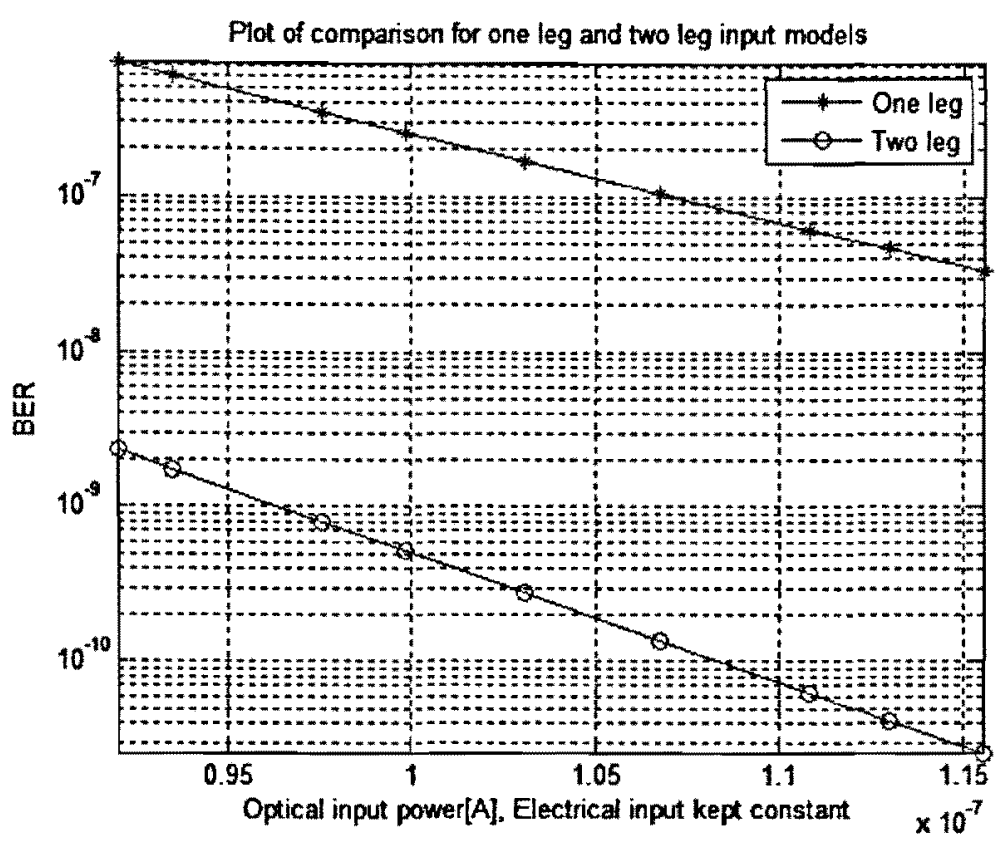

Figure 6.18: BER plot for performance comparison of the two models with no thermal noise. Both the input signals are kept at equal strength and fixed electrical power level.

is remarkabely better for all the values at which the measurments are taken.

For Figure 6.19 the values of the launching optical power are indicated in unit of current. The BER is determined by sending $0.52 \mu \mathrm{A}$ to $0.72 \mu \mathrm{A}$ optical signal power. The half wave voltage is kept at $2 \mathrm{~V}$ and the amplitude of the electrical power is kept at $1 \mathrm{~V}$ level. The responsivity of the detecor is assumed 0.90. For this particular measurment bandwidth is taken as $10 \mathrm{GHz}$. The thermal noise is included in this measurment. To creat a remarkable difference, thermal noise is assumed to be 10 fold of the quantum noise. As can be observed in the graph that the two leg model is remarkabely better for all the values of launching optical power at which the measurments are taken. The difference in the BER curves increases as the transmission power increased. The BER has shift upward (as compared to the no thermal noise plot) for both models as expected from the incorporation of significant amount of thermal noise. 


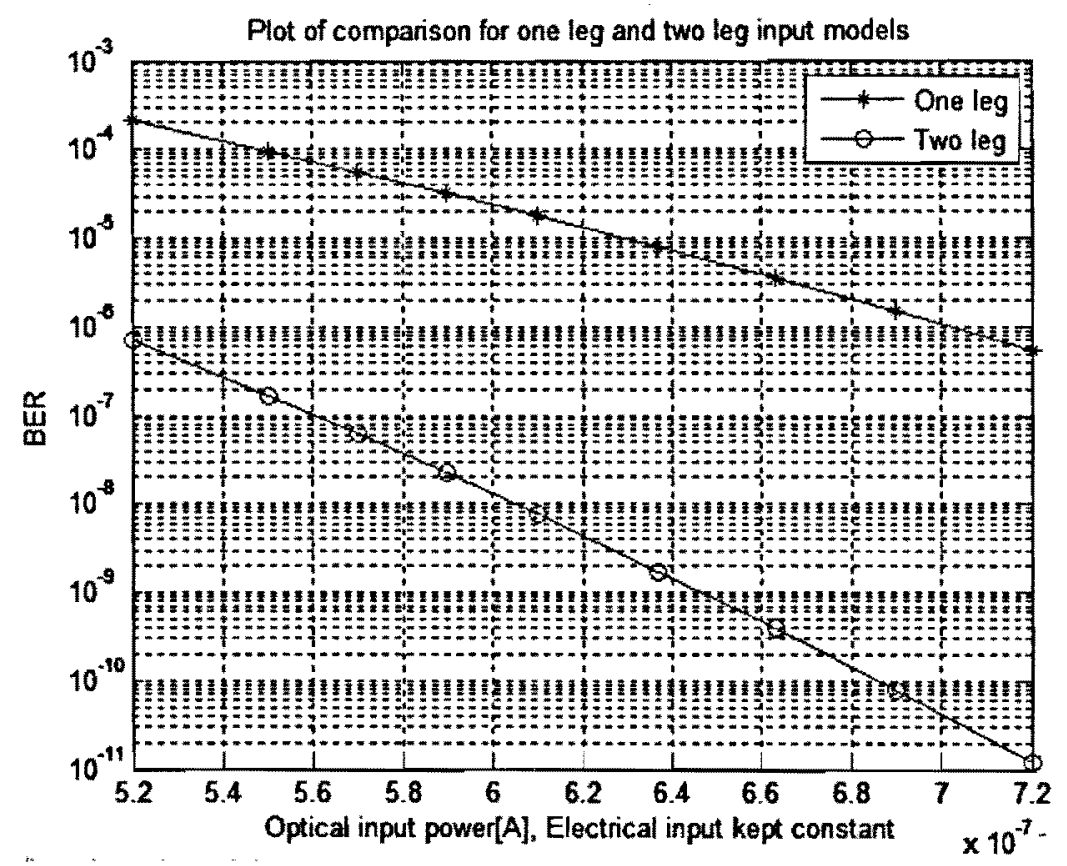

Figure 6.19: BER plot for performance comparison of the two models with thermal noise. Both the input signals are kept at equal strength and fixed electrical power level. 


\section{Chapter 7}

\section{Conclusion and Recommendation}

\subsection{Conclusion}

Optical fiber network is the solution for the futuristic high bandwidth demand. Several network services are supported by fiber optic links. FTTH extends the coverage of optical fibers from central station to home of subscriber. In order to make use of large band width capability of optical fiber, baseband services of cable TV, Internet and home phone are now provided by fiber optic networks through FTTH systems. The development of PON and its standardization activated the development and deployment of FTTH. RoF is another attractive application of fiber optic networks. RoF facilitates wireless access by exploiting the large bandwidth capacity of optical fiber and reducing the number of expensive wireless devices involved. RoF takes the low attenuation advantage of fiber optics and could be applied to less accessible and obstructed areas.

Integrating two or more network services has the benefit of reusing the deployed infrastructure for additional services and reduces overhead cost for each deployment and operation. Hybrid access network of integrated RoF and FTTH have a high possibility of driving future network trend. It reduces network implementation cost for service providers as wired and wireless services are delivered through same fiber network providing high network availability to meet future customer demands. Integrating of these services involves interleaving the service signals, modulating to optical carrier, transmitting the interleaved signal, detecting at the remote station and separating the different components at the destinations. 
Motivated by the integration of FTTH and RoF systems, this project has been put together, developed and accomplished simulation of integrating two optical based services. The simulation set up required a thorough understanding both Simulink and MATLAB software packages. It is observed that the signal source simulators, mixing simulators, filtering simulators and other process simulating devices have to be programmed precisely in order to get a meaningful result. The signal source simulators have to be adjusted for sample size that is large enough to observe the expected result. However, too much samples from these simulators might increase the computer processing load and lead to extremely extended simulation time.

Since the Simulink simulation for several processes took place simultaneously, both the service signals simulated had always been $\mathrm{ON}$ for the same span of signal time. In this project care had been taken to specify the time span that is large enough to accommodate the width of both the signals required for the simulation purpose. Real time simulation for the three phase processes(Simulink $\rightarrow$ MATLAB $\rightarrow$ Simulink) is unachievable as the modulation of optical signal requires extremely large sample size in the MATLAB stage. This is due to the fact that the optical signal frequency is in the range of $100 \mathrm{THz}$ while the electrical signal is in a few $\mathrm{GHz}$ range. So modulating the optical signal by electrical signal requires the multiplication of the electrical signal sample by a factor of 100,000 . This causes extremely large sample requirement at the MATLAB stage. In order to cope up this problem, the low pass signal equivalent for optical carrier is assumed. This can effectively reduce the sample size of the simulation processes.

In this project two models for the modulation of optical signal by electrical messages has been developed. Two mathematical equations that represent each MZM modulation have been formulated and provided. In order to compare the models performance, BER plots for each model at different scenarios of input was built. The BER plots indicated that the two leg input model is better in performance for all sets of input signal characteristics considered. Moreover, the mathematical derivation shows that one leg input model constitute several frequency components other than the actual message signal frequencies. This in turn causes additional interferences within the optical fiber 
as a consequence of dispersion.

This project can also be extended to compare the effect of change with performance of two power launching schemes in the RoF system. It can be used as comparing the two models with two separate services, by keeping the optical power level at a constant value and changing the electrical signal strength and determining for the optimal operational requirement. In the other case, it can be used to compare the two models by keeping all the input electrical signals at a fixed power level and look for optimal operation scenarios by changing the optical power strength and other important design parameters in the optical configuration.

\subsection{Future Work}

For future works, the simulation result can be enhanced by better implementation of the capabilities of Simulink and MATLAB, especially in RF-communication tool kits. I also suggests that simulation work could be better handled by dedicated optical simulation software. Closer investigation can be done to find out whether SSB or DSB can handle the modulation better. In other words, instead of using DSB, it is desirable to see if using SSB modulation schemes for both models might reduce the difference between the two model performances and yield a better comparison schedule. Since SSB consideration reduce the dispersion effect and increase the spectral efficiency, it would be a better consideration for single leg model as it suffers the most by dispersion 


\section{References}

[1] J.J. Vegas Olmos, T. Kuri, T. Sono, K. Tamura, H. Toda, and K.-i. Kitayama, " Reconfigurable 2.5-Gb/s Baseband and 60-GHz (155-Mb/s) Millimeter-Waveband Radio-Over- Fiber (Interleaving) Access Network," IEEE Journal of Light Wave Technology, Vol. 26, No. 15, pp. 2506- 2512, Aug. 2008.

[2] T. Kuri, K. - i. Kitayama, and C.P. Tsekrekos, "Simultaneous Transmission of Millimeter-Wave and Baseband Signals Using Wavelength Interleaving and Polarization Multiplexing for an Integrated Reconfigurable Access Network," IEEE, Photonics Technology Letters, Vol. 21, No. 21, pp. 1597- 1599, 2009.

[3] L. Chun-Ting, J. Chen, P. Peng-Chun, P. Cheng-Feng, P. Wei-Ren, C.Bi-Shiou, and S. Chi, "Hybrid Optical Access Network Integrating Fiber-to-the-Home and Radio-Over-Fiber Systems," IEEE Photonics Technology Letters, Vol. 19, No. 8, pp. 610-612, April 2007.

[4] T. Zhenning, "Improvements to Digital Carrier Phase recovery algorithm for high performance optical coherent receivers," Oct. 2010.

[5] L. Xiang, D.M. Gill, "Multi-carrier coherent receiver based on a shared optical hybrid and a cyclic AWG Array for Terabit/s optical transmission," June 2010.

[6] G. Paul E, "Fiber To The Home, The New Empowerment," WILEYINTERSCIENCE, A John Wiley \& Sons, inc., Publication, 2006.

[7] D.J. Santos, and F.J. Fraile-Pelaez, "Pulse normalization in optical receiver shotnoise performance," Optoelectronics IEEE Proceedings, Vol. 146, No. 4, pp. 201, Aug. 1999. 
[8] B. John R, and A.L. Edward, " Performance of Coherent optical receivers," Aug. 1990.

[9] J. Wen-Jr, L. Chun-Ting, S. Po-Tsung, H. Li-Ying Wang, C.Jyehong, and C. Sien, "Simultaneous Generation and Transmission of $60-\mathrm{GHz}$ Wireless and Baseband Wireline Signals With Uplink Transmission Using an RSOA," IEEE Photonics Technology Letters, Vol. 22, No. 15, pp. 1099, Aug. 2010.

[10] M. John Senior, and M. Yousif Jamro, "Optical Communications Principles and Practice," Pearson Education Limited, Third Edition, 2009.

[11] P. Almeida, and H. Silva, "Multiservices and Multiband Optical Signal Generation for Hybrid Access Networks.", IEEE Optical Network Design and Modeling $(O N D M)$, pp 1-6, 2011.

[12] C.P. Tsekrekos, T. Kuri, and K.-i Kitayama, "Distribution of Millimeter-Wave and Baseband Services Over an Integrated Reconfigurable Access Network Platform," IEEE Light wave Technology Journal, Vol. 28, No. 19, pp. 2783-2790, 2010.

[13] L. Wen-Yi, D. Hsiang-Chun, L. Chung- Yi, S. Heng Sheng, C. Kuo - Hsiang, and L. Hai -Han, "Fiber-To-The-Home/Radio-Over-Fiber Transport Systems, " IEEE Optoelectronics and Communication conference, pp. 1, July 2009.

[14] L. Chun-Ting, P. Wei-Ren, P. Peng-Chun, P. Cheng Feng, C. Bi- Shio, and S. Chi, "Simultaneous Generation of Baseband and Radio Signals Using Only One Single- Electrode Mach - Zehnder Modulator With Enhanced Linearity," IEEE Photonics Technology Letters, Vol.18, No.23, pp. 2481, Dec. 2006.

[15] K. Gerd, "Optical Fiber Communications, " McGraw-Hill Series in Electrical and Computer Engineering, Third Edition, 2000.

[16] L. Chinlon, "Broadband Optical Access Networks and Fiber-to-the-Home, "John Wiley ES Sons, Ltd, 2006

[17] C.K. Calvin, "Optical Performance Monitoring," ELSEVEIR Inc., 2010.

[18] B. Le Nguyen, "Digital Optical Communications," CRC Press, Tayler \& Francis Group, 2009. 
[19] L. Yuyu, and Y. Huazhong, "High-Speed Optical Transceivers, Integrated Circuits Designs and Optical Devices Techniques," World Scientific Publishing Co. Pte. Ltd., 2006.

[20] B. Michael, W. Eric, and S. Van, "Fiber Optics Handbook," McGraw-Hill, 2002.

[21] G.E. Stillman, L.W. Cook, G.E. Bulman, N. Tabatabaie, R. Chin, and P.D. Dapkus, "Long- wavelength (1.3- to $1.6 \mu \mathrm{m})$ detectors for fiber-optical communications," Electron Devices IEEE Transactions , Vol. 29 , No. 9, pp. 1355, Sep. 1982.

[22] K. Kikuchi, "Proposal and performance analysis of novel optical homodyne receiver having an optical preamplifier for achieving the receiver sensitivity beyond the shot-noise limit," IEEE Photonics Technology Letters, Vol. 4, No. 2, pp. 195, Feb. 1992.

[23] B. Kanchan, "An overview of passive optical networks and components, " Ryerson University, Electrical and Computer Engineering, 2007.

[24] K. Souheil, " Passive Optical Networks and FTTx: Technology and solutions," Ryerson University, Electrical and Computer Engineering, 2007.

[25] B. Ng, M.S. Ab-Rahman, A. Premadi, and K. Jumari, "Portable Network Monitoring System for Passive Optical Network (PON), "IEEE Computer Technology and Development, 2009. ICCTD '09. International Conference, Vol. 2, pp. 176, Nov. 2009.

[26] S.Yika, and C. Qingjiang, "Convergence of RoF and access systems employing dual parallel modulator in the central station," IEEE Optical Fiber Communication and Optoelectronics Conference, 2007 Asia, pp. 310 - 312, Oct. 2007.

[27] L. Hai-Han, M. Hsien-Li, and A.S. Patra, "Radio-on-Fiber Transport Systems Integration with $622-\mathrm{Mb} / \mathrm{s}$ Baseband Transmission , IEEE Photonics Technology Letters, Vol. 20 , No.19, pp. 1618 - 1620, Oct. 2008.

[28] K. Mark, F.M. Nan, H.R. Scott, R. Charles, and F. Claudia, "Dispersion- Induced Power Penalty in Fiber-Bragg-Grating WDM Filter Cascades Using Optically Preamplified and Non preamplified Receivers," IEEE Photonics Technology Letters, Vol. 12, No. 10, pp. 1406, Oct. 2000. 
[29] NEC corporation, Minato ku, Tokyo, Japan. [http://www.nec.co.jp/press/en/0903/1901.html.]

[30] Telecommunication Market Research; Totel Pty Ltd, Booval, Australia. [ http://www.totel.com.au/north-america-telecommunications-research.aspcid \& toc $=\mathrm{US}=3608$.]

[31] H. Stephen, "FTTH; Deployment Spreads Globally," lyLightwave Pennwell Corp. 98 Spit Brook Road Nashua, NH 03062, March 2008. [http://www.lightwaveonline.com/business/market-research/ftth-councilsdeployment-spreads-globally- $54885252 . \mathrm{html}$.]

[32] N. Pleros, K. Vyrsokinos, K. Tsagkaris, and N.D. Tselikas, "A 60 GHz RadioOver-Fiber network Network Architecture for Seamless Communication With High Mobility Light wave Technology,"IEEE Light wave Technology Journal of Issue, Vol. 27, No. 12, pp. 1957 - 1967, June 2009.

[33] S.A.C.San Borja, Peru Telmark, Lima-Peru. [http://www.telmarkperu.com/Documentos/GPON.pdf.]

[34] Y. Tetsuy, "NTT Tsukuba Forum 2006 Workshop lectures,", Nippon Telegraph and Telephone Corporation, 3-1, Otemachi 2-chome, Chiyoda-ku, Tokyo 100-8116, Japan., 2006 [http://www.ntt.co.jp/about _ e/corporatedata.html.]

[35] Fiberfab US 52745 Drafter Rd. La Pine, Oregon 97739. [http://www.fiberfab.us/contact.html.]

[36] M. Jose,and S.D. Pinto, "Evolution-PON-standards", [http://code.ua.pt/attachments/download/1545.]

[37] Cisco Public Information, "Fiber Types in Gigabit Optical Communications, " Cisco Systems, Inc. 170 West Tasman Dr. San Jose, CA 95134 USA, pp. 4, 2008. [http://www.cisco.com/en/US/prod/collateral/modules/ps5455/images /white_paper_c11-463661-07.jpg.] 
[38] D. Stephen, "FTTH: A necessary Future, " Opterex, 503 Olde Waterford Way, Leland, NC 28451, USA, . [http://www.opterex.com/.]

[39] J. M. B. Oliveira, H.M. Salgado, M.R.D Rodrigues, "Large Signal of Mach -Zehnder Modulator Intensity Response in a Linear Dispersive Fiber," Instituto de Engenharia de Sistemas e Computadores do Porto, Porto, Portugal. [http://citeseerx.ist.psu.edu/viewdoc/summary?doi=10.1.1.149.1060.]

[40] J. Rodriguez-Asomoza, C. Gutierrez-Martinez, R. Rosas-Romero, R. AlejosPalomares, J.A. Navarro-Martinez, S. Lobato-Larios, O.E. Morales-Hernandez, D. Alfaro-Cordova, and I. Zaldivar-Huerta, " Detection-transmission of electric signals using an optical Mach-Zehnder modulator and dispersive optical fiber channel, " IEEE Instrumentation and Measuremen Technology Conference, Vol. 3, pp. 1961 1964, May 2005.

[41] M. Biagio, and Z. Xiupu, "Linearized Optical Single-Sideband MachZehnder Modulator for Radio-Over-Fiber Systems, "IEEE Photonic Technology Letters, Vol. 19, No. 24, Dec. 2007.

[42] J. Wen-Jr, L.Chun-Ting, N. Anthony, S.Po-Tsung, C. Jason (Jyehong), S. Michael, A. Frank, and C. Sien, "Simple 14-Gb/s Short-Range Radio-Over-Fiber System Employing a Single-Electrode MZM for $60-\mathrm{GHz}$ Wireless Applications," IEEE Journal of Lightwave Technology, Vol. 28, No. 16, pp. 2238-2246, Aug. 2010.

[43] C. Xiaogang, and H. Dexiu, " Effect of Mach-Zehnder Modulator DC Extinction Ratio on Single Sideband Modulation Radio over Fiber Link," IEEE Communications and Photonics Conference and Exhibition, Vol. 2009-suppliment, pp. 1-6, Nov. 2009.

[44] G. E. James, "Bit-Error-Rate Simulation Using Matlab," Transcript International, Inc., Aug. 2003.

[http://www.efjohnsontechnologies.com/resources/dyn/files/75831/_fn/bit-errorrate.] 
[45] N. Brett, "Spectral Analysis using the FFT," Department of Electrical and Computer Engineering The University of Newcastle, Australia. [http://sigpromu.org/brett/elec2400/matlab3.pdf.]

[46] H. Rongqing, and O.S. Maurice, "Fiber optic measurement techniques," Elsevier Academic Press, 30 Corporate Drive, Burlington, MA 01803, USA, 2009. [www.elsevier.com.]

[47] K. Toshiaki, K. Ken-ichi, S.Andreas, and O. Yasunori, "Fiber-Optic MillimeterWave Downlink System Using $60 \mathrm{GHz}$-Band External Modulation," IEEE Journal of Lightwave Technology, Journal of, vol. 17, no. 5, pp. 799-806, Aug. 2002.

[48] D. Remondo, M. Nunes, S. Sargento, M. Cesana, I. Filippini, J. Triay, A. Agusti, M. De Andrade, Ll. Gutierrez, S. Sallent, and C. Cervello-Pastor, "Integration of Optical and Wireless Technologies in the Metro-Access: QoS Support and Mobility Aspects, "IEEE, Next Generation Internet Networks, NGI '09, pp. 1- 8, July 2009.

[49] Q.Xiaoqiong, L. Jiaming, Z. Xiaoping, X. Liang, "Fiber Dispersion and Nonlinearity Influences on Transmissions of AM and FM Data Modulation Signals in Radio-Over-Fiber System," IEEE Journal of Quantum Electronics, Vol. 46, No. 8, pp. 1170-1177, Aug. 2010

[50] S. Poompat, and M. Muriel, "Guaranteeing the BER in Transparent Optical Networks Using OOK Signaling," IEEE Journal on Selected area in Communication, Vol. 20, No. 4, pp. $789-799$, May 2002.

[51] L. Chris, C.C. John, J. Yu, "Modulation Characteristics of Semiconductor MachZehnder Optical Modulators," IEEE Journal of Lightwave Technology, Vol. 15, No. 4, pp. 697 - 703, Apr. 1997. 


\section{Appendix A}

\section{Simulink Devices and Tools Setup}

1) $622 \mathrm{Mb} / \mathrm{s}$ digital signal simulator:

Bernoulli binary generator 1

Probability of zero $=0.25$

Initial seed $=61$

Sample time $=1.6077 \mathrm{e}-9$

Interpret vector parameter $=$ select

Output data type $=$ double

2) Band limited white noise simulator:

Noise power $=[0.001]$

Sample time $=0.1$

Seed $=61$

Interpret vector parameter $=$ select

3) $25 \mathrm{GHz}$ carrier sinusoidal simulator:\}

Waveform $=$ sine

Time $(t)=$ use simulation time

Amplitude $=1$

Frequency $=25 \mathrm{e} 9$

Unit $=$ Hertz

Interpret vector parameter $=$ select

Adder uses sample time inherited (-1 selected)

4) Mixers:

Number of input $=2$

Sample time $=5 \mathrm{e}-12$

5) $1.25 \mathrm{~Gb} / \mathrm{s}$ digital signal simulator:

Bernoulli binary generator 2 
Probability of zero $=0.25$

Initial seed $=61$

Sample time $=8 \mathrm{e}-10$

Interpret vector parameter $=$ select

Output data type $=$ double

\section{6) $5 \mathrm{GHz}$ carrier sinusoidal simulator:}

Waveform $=$ sine

Time $(t)=$ use simulation time

Amplitude $=1$

Frequency $=5 \mathrm{e} 9$

Unit $=$ Hertz

Interpret vector parameter $=$ select

\section{7) Eye diagram scope:}

Sample per symbol $=8$

Offset $=0$

Symbol per trace $=1$

\section{8) B-FFT display}

Spectrum units $\mathrm{dBm} / \mathrm{Hertz}$

Spectrum type two sided(-Fs/2 ... Fs/2)

Buffer input $=$ on

Buffer size 128

Display property $=$ Show grid $/$ frame number

Axis property $=$ inherit sample time $/ 0 \mathrm{~Hz}$ offset

Frequency display time $=$ Auto

$\operatorname{Min}=150 \mathrm{dBm}$

$\operatorname{Max}=0 \mathrm{dbm}$

Magnitude $=$ square $\mathrm{dB}$ 


\section{Appendix B}

\section{MATLAB Codes}

$\underline{\text { Program I, One leg input MZM simulation program }}$

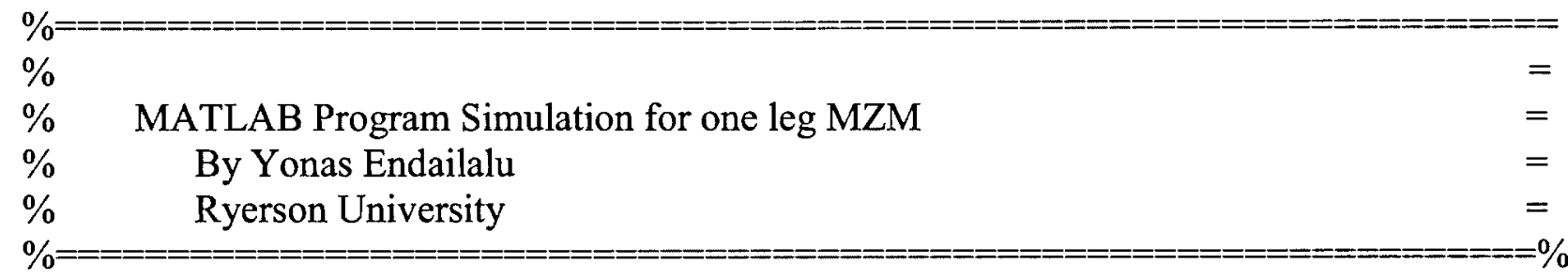

$\%$ Basic definition for variables and constants

$c=2.99793 \mathrm{E} 8 ; \%$ speed of light

$\mathrm{q}=1.60218 \mathrm{E}-19 ; \%$ charge of electron

$\mathrm{h}=6.62566 \mathrm{E}-34 ; \%$ Plank's constant

$\mathrm{Kb}=1.38054 \mathrm{E}-23$; \% Boltzman's constant

lambda = 1552e-9;

$\%=$

Imported data from simulink

$\%=$

$\%$ Importing radio 1 simulation result data

$\mathrm{SR}=$ load('onelegdata.mat')

oneleg_time $=$ SR.oneleg.Time;

oneleg_data = SR.oneleg.Data;

oneleg_length = length(SR.oneleg.Time);

$\%$ Importing radio 2 simulation result data

$\mathrm{SB}=$ load('onelegbasedata1.mat')

Baseband time = SB.onelegbase. Time;

Baseband_data = SB.onelegbase.Data;

Baseband_length = length(SB.onelegbase.Time);

$\%============$
$\%$ oneleg signal extractor

oneleg_word = zeros $(1,20)$;

for $\mathrm{k}=1: 20$;

for $\mathrm{i}=((\mathrm{k}-1) * 200)+1: \mathrm{k}^{*} 200$ 


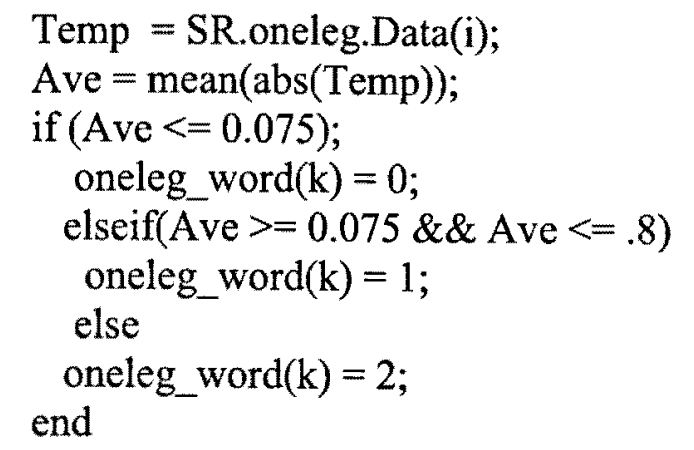

end

end

$\%=$

$\%$ oneleg Radio 1 signal extractor

onelegbase_word $=\operatorname{zeros}(1,20)$;

for $\mathrm{k}=1: 20$;

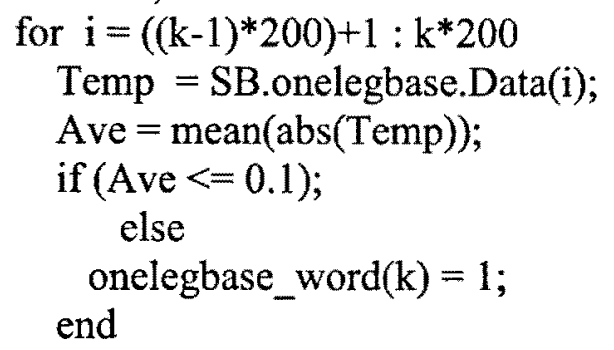

end

end

$\%===+===========$
$\%$ Radio 2 signal extractor

Radio_word = zeros $(1,10)$;

for $\mathrm{k}=1: 10$;

Ave $=($ oneleg_word $(2 * \mathrm{k}-1)+$ oneleg_word $(2 * \mathrm{k})) / 2$;

if (Ave $>=1.2$ )

Radio_word $(\mathrm{k})=1$;

elseif $(\overline{A v e}==1)$

if(onelegbase_word $(2 * k-1)==0)$;

Radio_word $(\bar{k})=1$;

end

else

Radio_word $(\mathrm{k})=0$;

end

end

$\%$ 


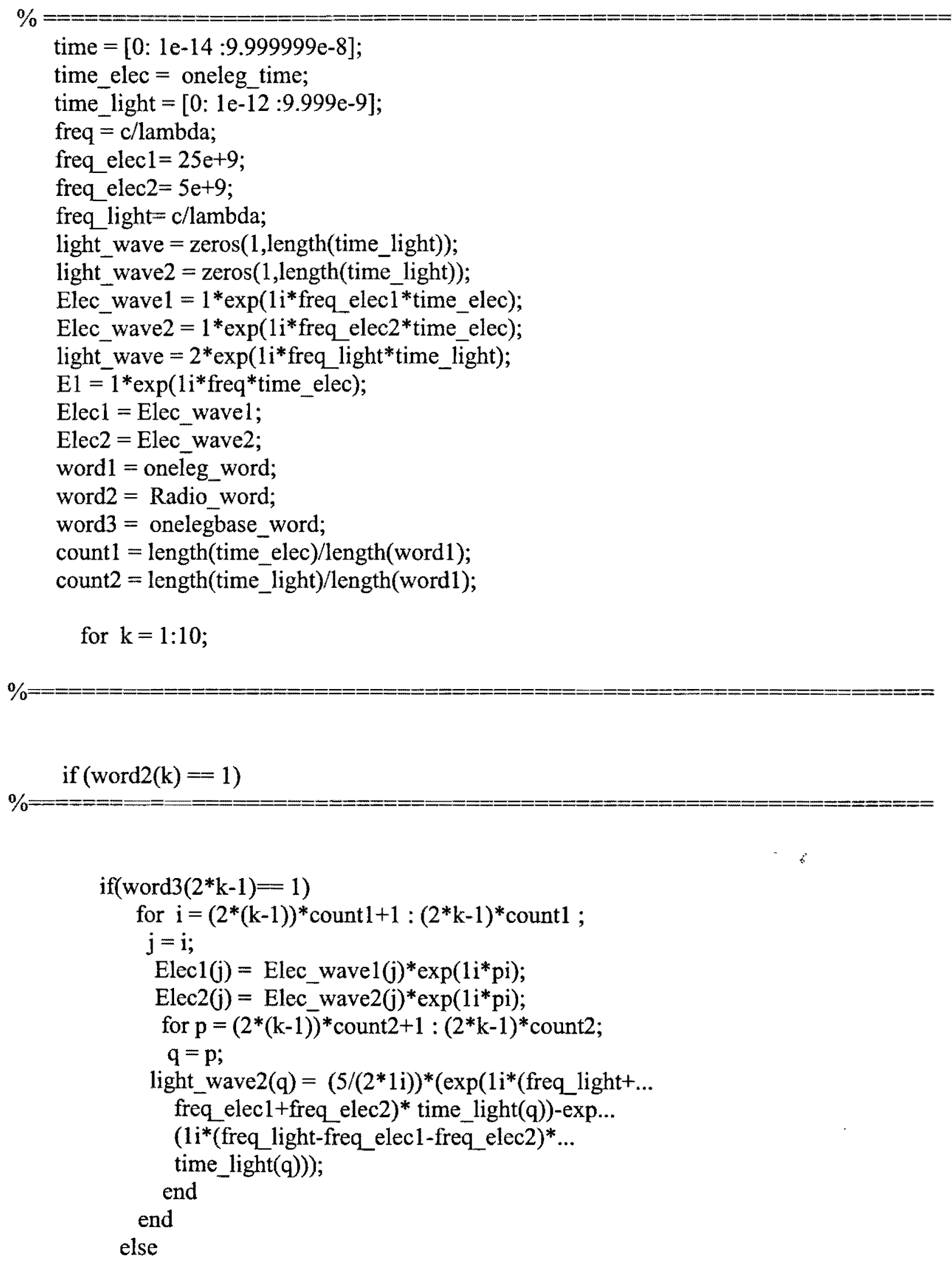




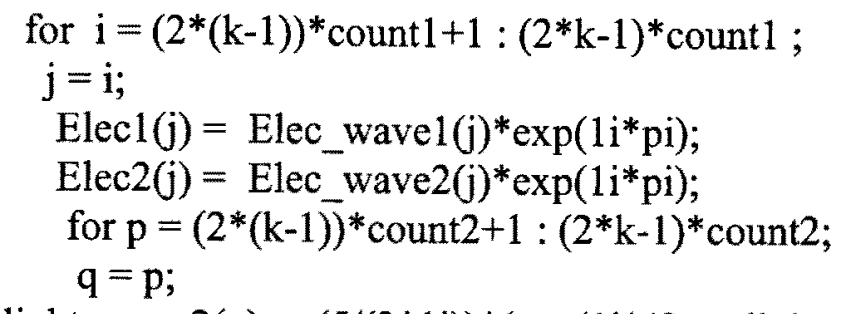

light_wave2(q) $=(5 /(2 * 1 i)) *\left(\exp \left(1 i^{*}(\right.\right.$ freq_light $+\ldots$

freq elec1)* time_light(q))-exp(1i*(freq_light-

freq_elec1)*time_light(q)));

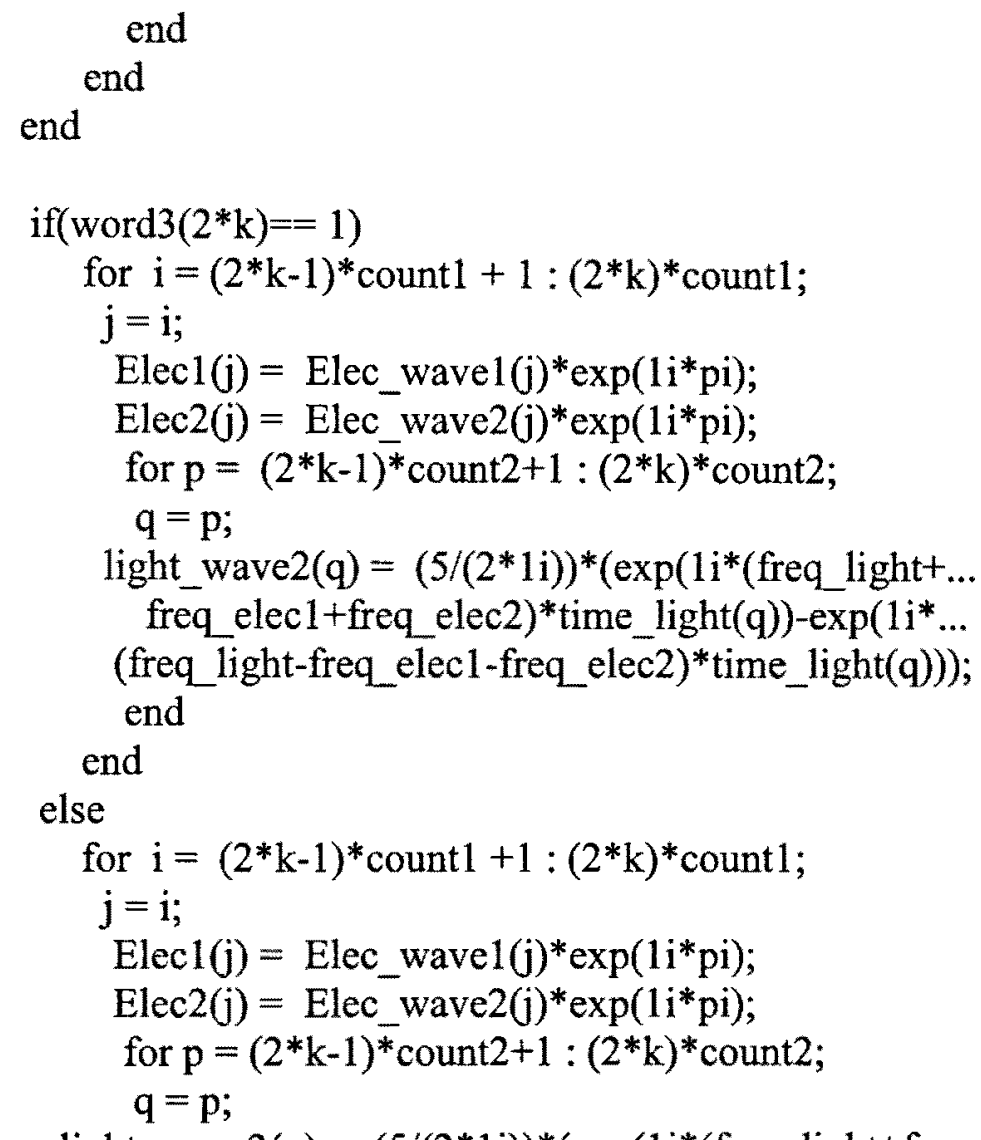

light_wave $2(\mathrm{q})=\left(5 /\left(2^{*} 1 \mathrm{i}\right)\right)^{*}\left(\exp \left(1 i^{*}(\text { freq_light }+ \text { freq_elec } 1)^{*} \ldots\right.\right.$

time_light(q))-exp(1i*(freq_light-freq_elec1)*time_light(q)));

end

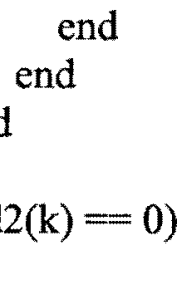

if $($ word $3(2 * k-1)=1)$ 


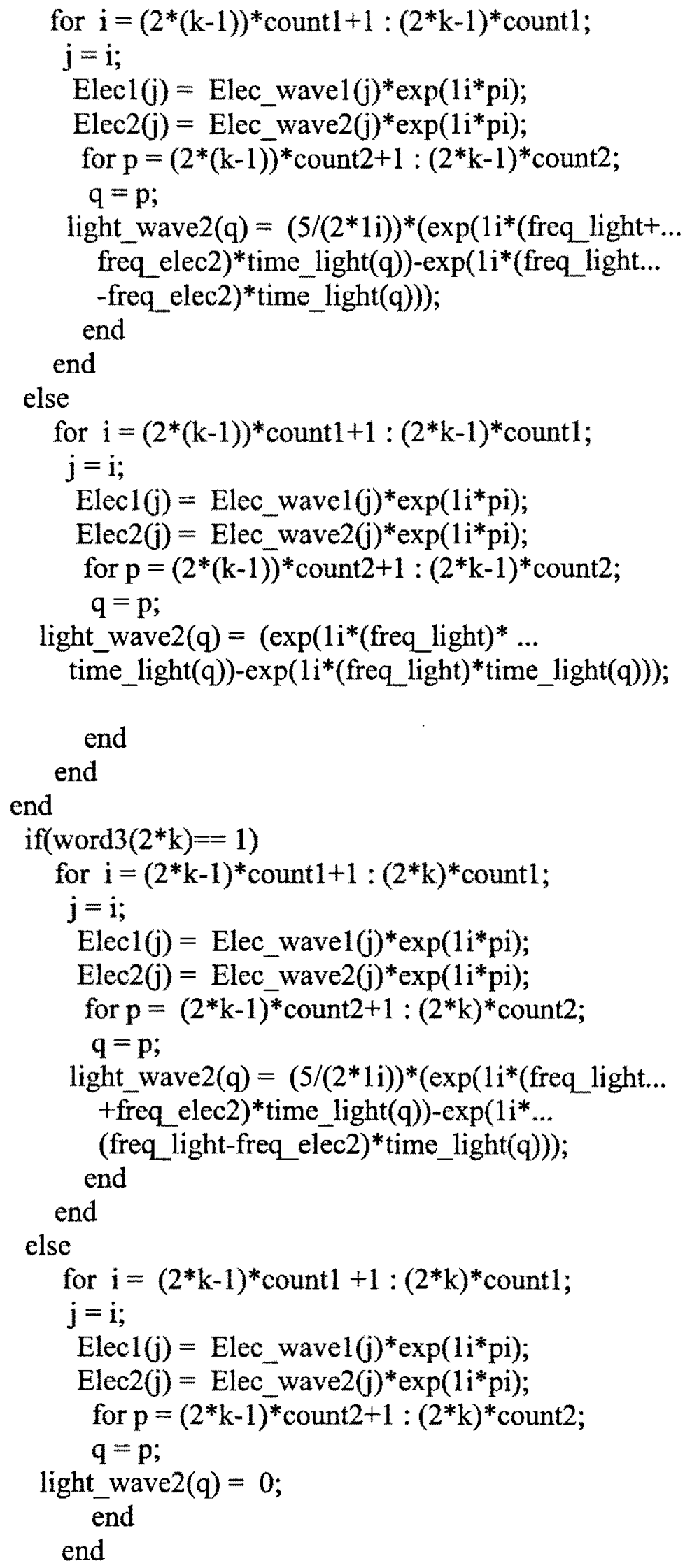


end

end

end

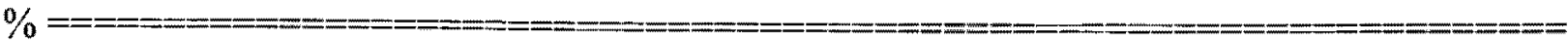

Eout $=$ Elec2 + Elec_wave1;

light_wave3 = light_wave + light_wave2;

$\%$ plot(time_elec, Eout, 'g',time_elec, Elec_wave, 'r'...

$\%$,time_elec, Elec2, 'b');

$\%$ plot(time_elec, Elec2, 'b');

plot(time_light, light_wave2, ' $\mathrm{b}^{\prime}$ );

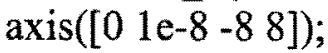

fft(light_wave2);

Program II, Two leg input MZM simulation program

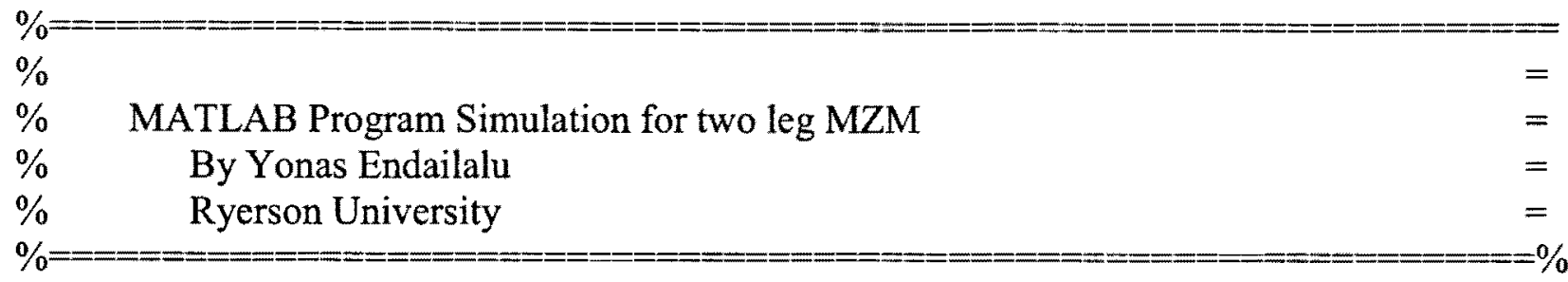

$\%$ Basic definition for variables and costants

$c=2.99793 \mathrm{E} 8 ; \%$ speed of light

$\mathrm{q}=1.60218 \mathrm{E}-19 ; \%$ charge of electron

$\mathrm{h}=6.62566 \mathrm{E}-34 ; \%$ Plank's constant

$\mathrm{Kb}=1.38054 \mathrm{E}-23$; \% Boltzman's constant

lambda $=1552 \mathrm{e}-9$;

$\%$

Imported data from simulink

$\%$

$\%$ Importing radio 1 simulation result data

$\mathrm{SR}=$ load('twolegradiol data.mat')

twolegradio_time $=$ SR.twolegradio.Time;

twolegradio_data $=$ SR.twolegradio.Data;

twolegradio_length = length(SR.twolegradio.Time);

$\%$ Importing radio 2 simulation result data

SB = load('twolegradio2data.mat')

twolegbaseband time $=$ SB.twolegbase.Time;

twolegbase_data = SB.twolegbase.Data;

twolegbase_length = length(SB.twolegbase.Time); 

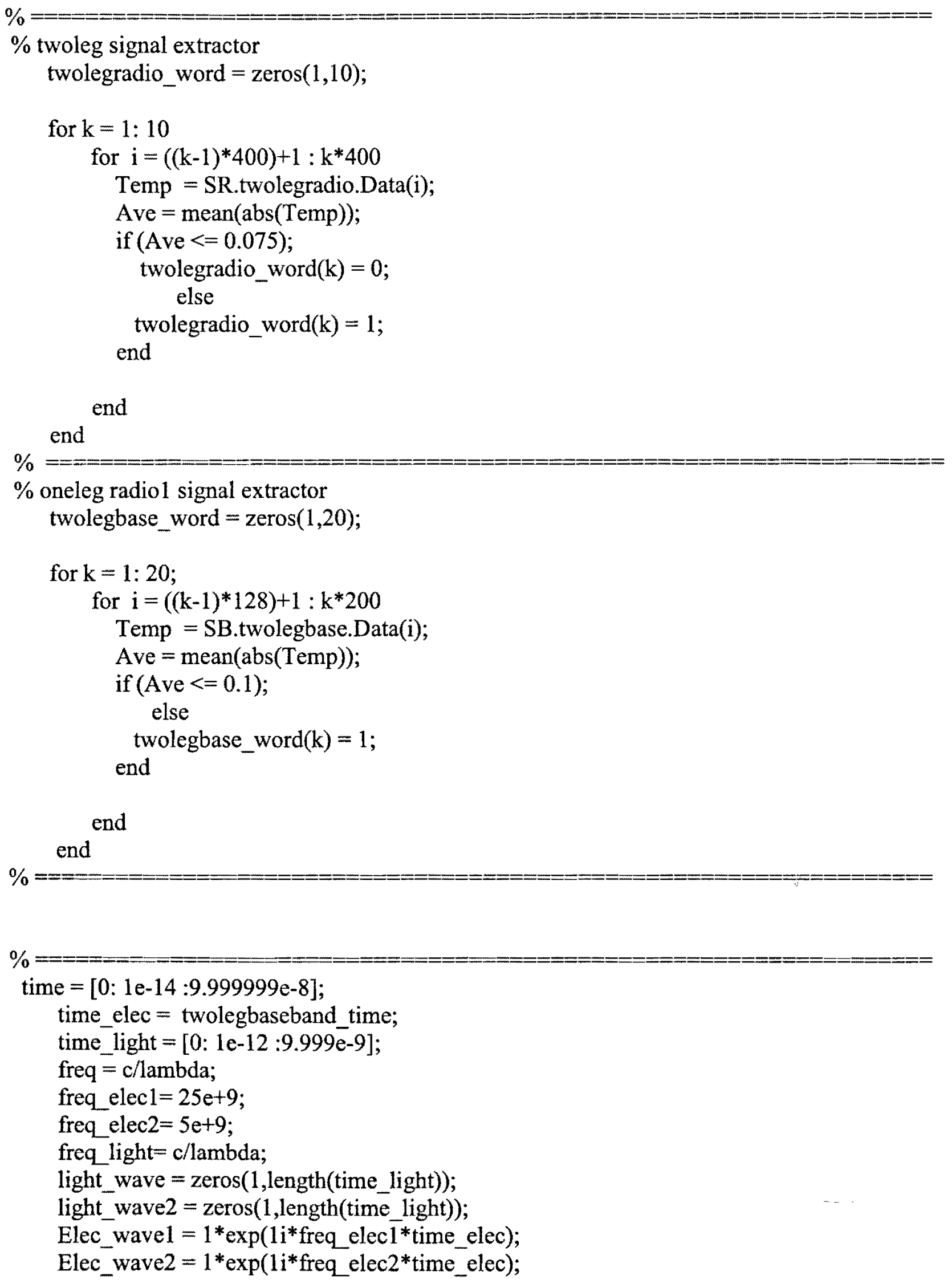
light_wave $=2 * \exp \left(1 \mathrm{i}^{*}\right.$ freq_light $*$ time_light $)$;

$\mathrm{El}=1 * \exp \left(1 \mathrm{i}^{*}\right.$ freq*time_elec $)$;

Elec1 $=$ Elec_wave 1

Elec2 = Elec_wave2;

word2 = twolegradio_word;

word3 = twolegbase word;

count 1 = length(time_elec)/length(word1);

count 2 = length(time_light)/length(word1);

for $\mathrm{k}=1: 10$;

$\%$

if $(\operatorname{word} 2(\mathrm{k})==1)$

$\%$

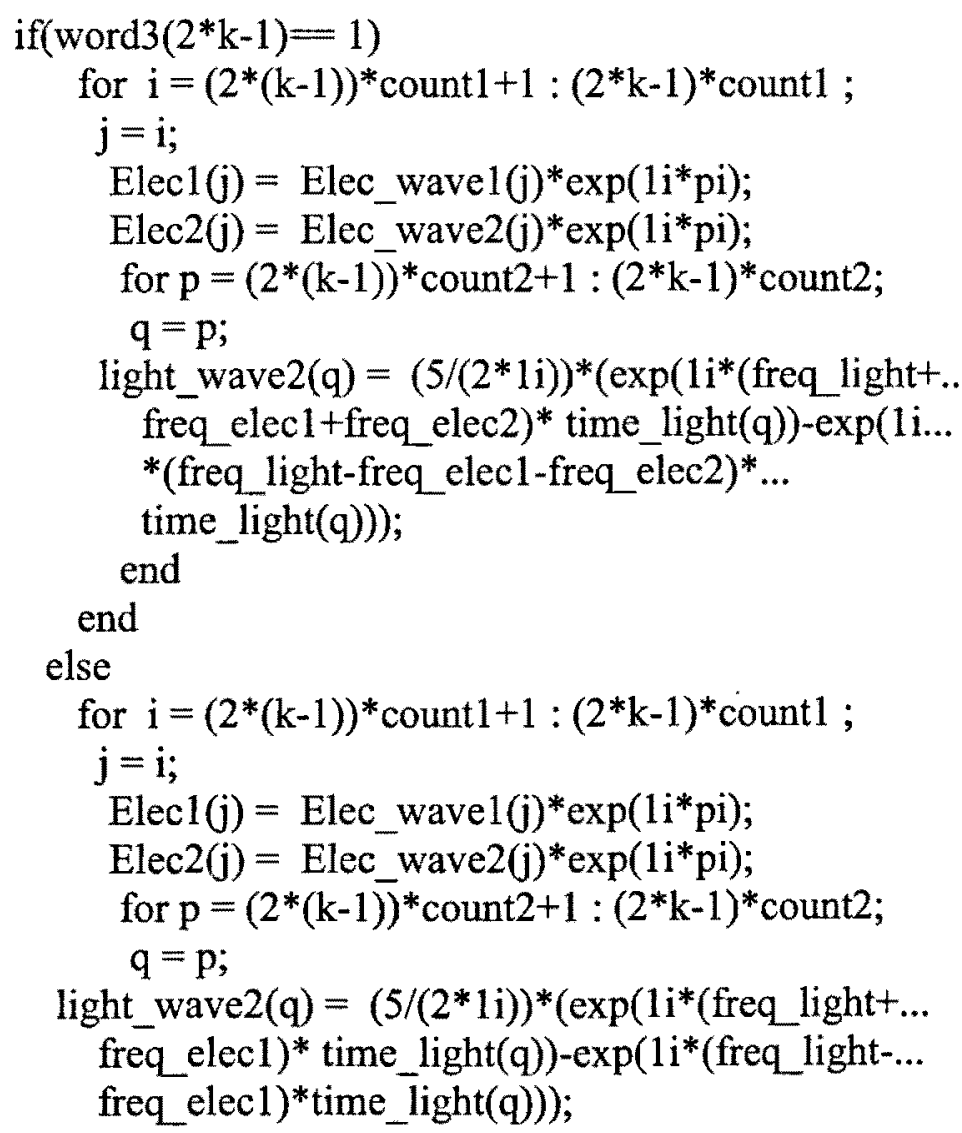



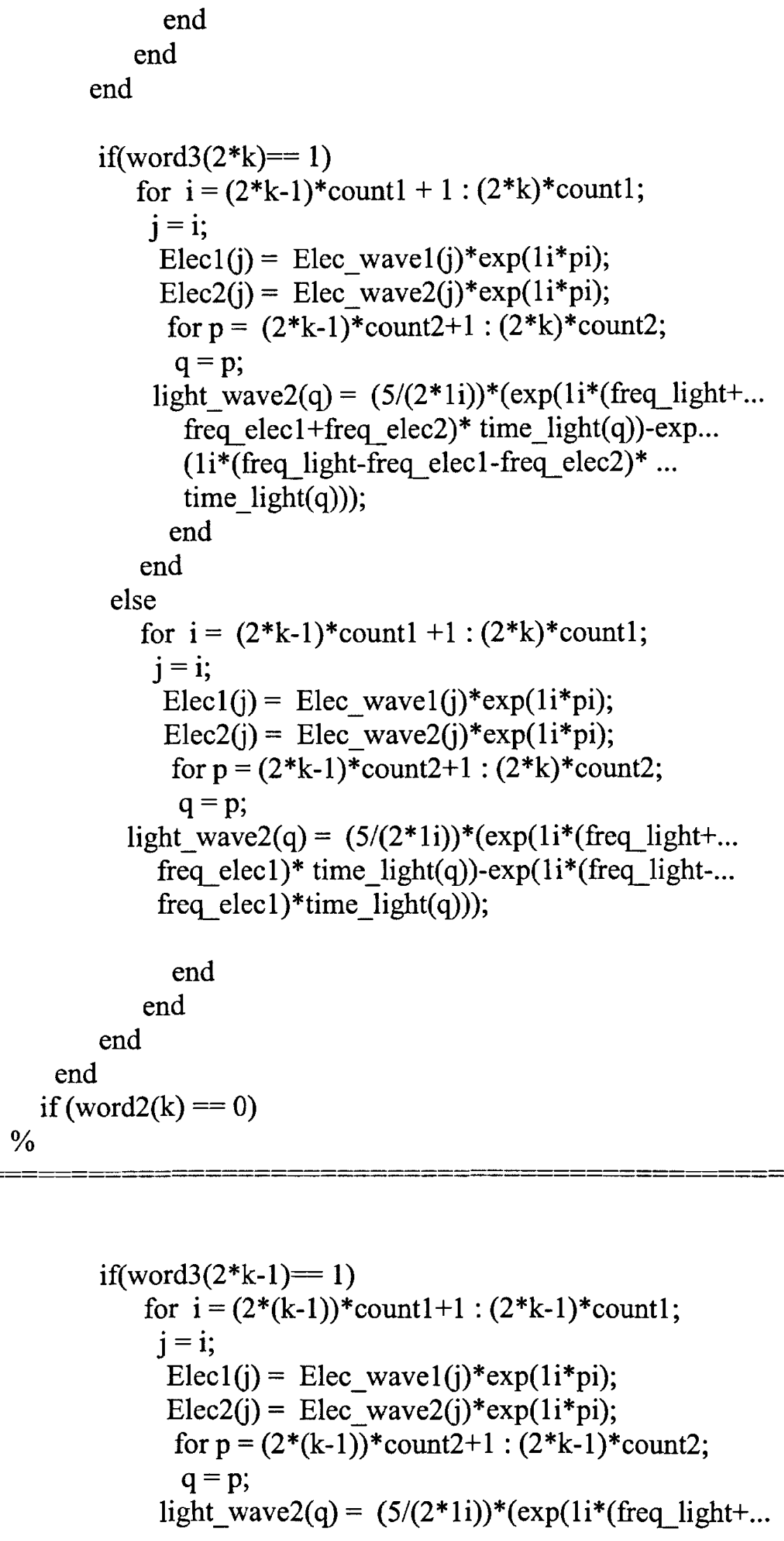

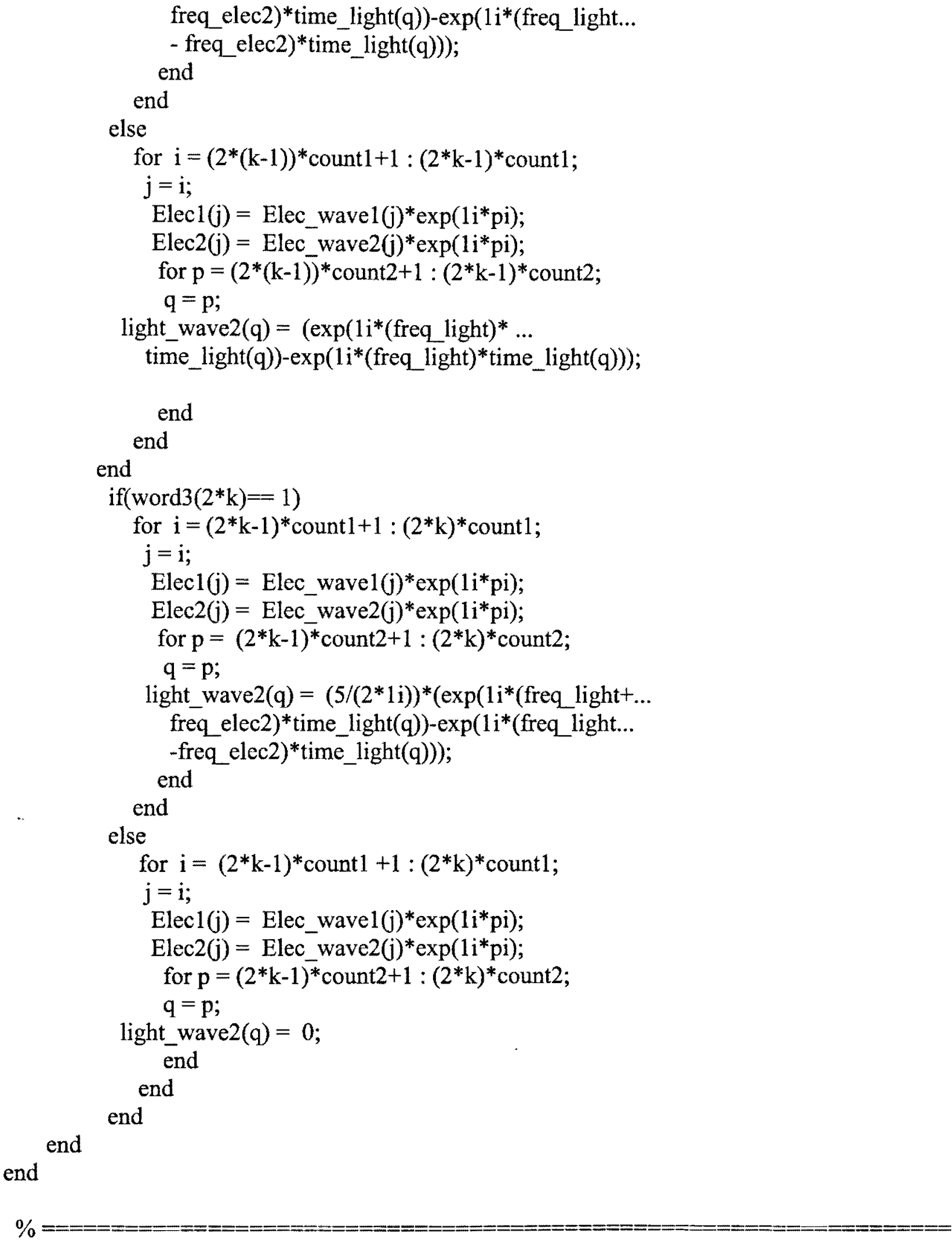

Eout $=$ Elec2 + Elec_wave1; 
light_wave3 = light_wave + light_wave2;

$\%$ plot(time_elec, Eout, 'g',time_elec, Elec_wave, 'r'...

$\%$,time_elec, Elec2, 'b');

$\%$ plot(time_elec, Elec2, 'b');

plot(time_light, light_wave2, 'b');

axis([0 $1 \mathrm{e}-\overline{8}-8 \mathrm{8}])$;

fft(light_wave2);

\section{Program III - BER PLOTTING PROGRAM}

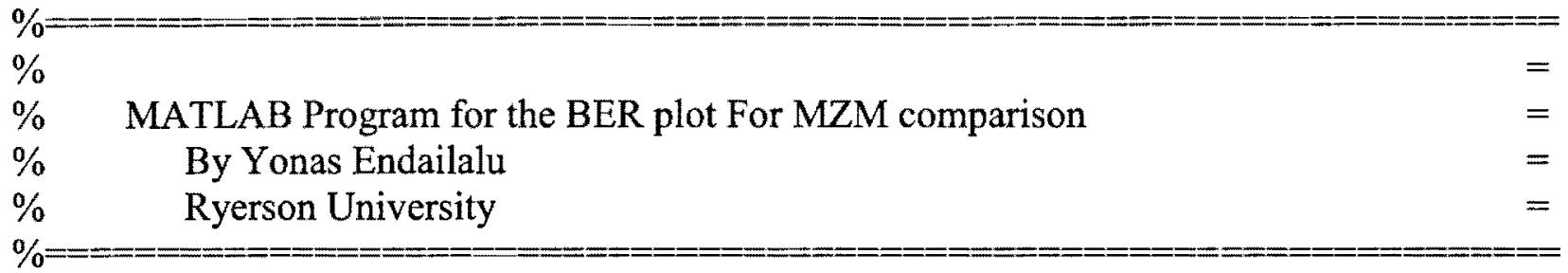

$\%$ Input voltage sample array for alpha and beta

One $=[11 ; 1.2 ; 1.2 ; 1.41 .4 ; 1.61 .6 ; 1.81 .8]$

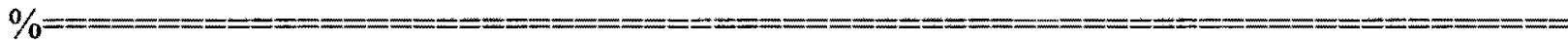

$\%$ Input value for half wave voltage (V-pi)

$$
\mathrm{V} \text { pi }=2
$$

$\%=====-======+=======-==$
eta $=$ pi $/ V$ pi $\%$ coefficient of phase change

$\mathrm{M} 1=0 ; \mathrm{a} \_$one $=0 ; \mathrm{b} \_$one $=0 ; \mathrm{c}$ _one $=0 ; \mathrm{d} \_$one $=0 ; \mathrm{e} \_$one $=0 ; \mathrm{f}$ _one $=0 ;$

$\%$ Bessel function evaluation for the six coefficient parameters

for $\mathrm{i}=1: 5$;

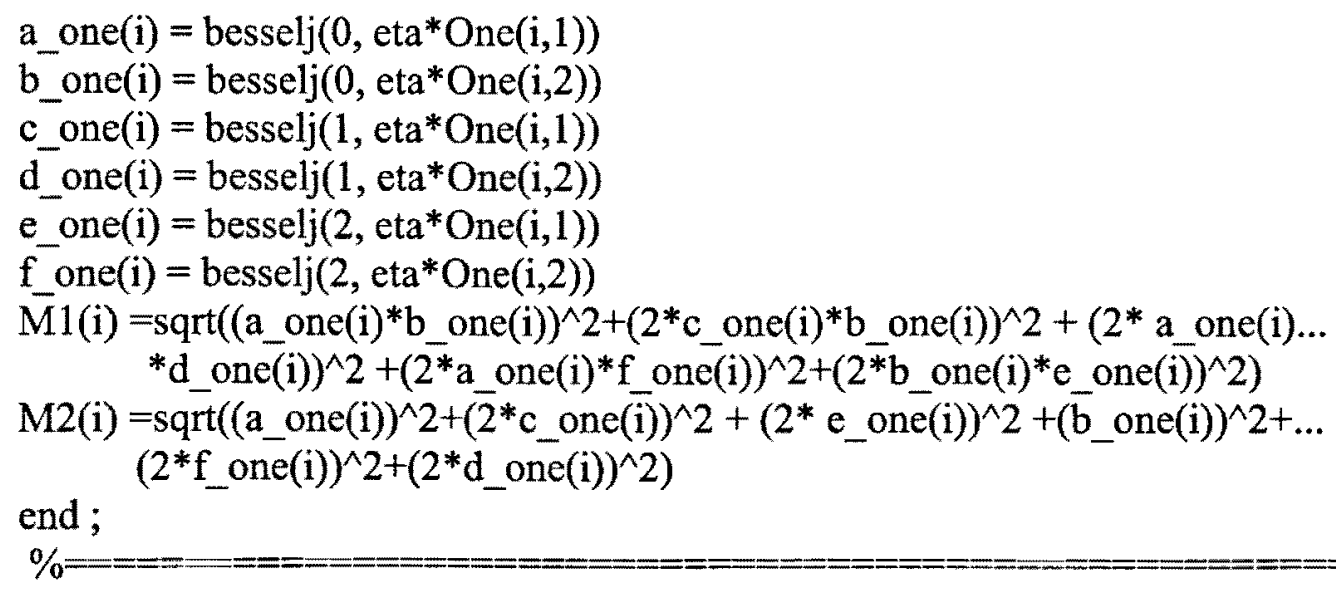


ip_A $=\mathrm{M} 1 * 1.38 \mathrm{e}-7 ; \%$ Input optical current

ip_B $=\mathrm{M} 2 * 1.38 \mathrm{e}-7 ; \%$ Input optical current

SNR A $=$ ip A $\mathrm{A} /(1.4142 * 1.6 \mathrm{e}-19 * 6 \mathrm{e} 9) ; \%$ SNR calculation

SNR_B $=$ ip_B $/(1.4142 * 1.6 \mathrm{e}-19 * 6 \mathrm{e} 9) ; \%$ SNR calculation

SNR $\mathrm{dBA}=10 * \log 10(\mathrm{SNR} A)$;

SNR dBB $=10 * \log 10($ SNR $B)$;

BER_A $=(1 / 2) *\left(\operatorname{erfc}\left(\left((\right.\right.\right.$ SNR_A $\left.\left.\left.){ }^{\wedge} 0.5\right) /(2 * \operatorname{sqrt}(2))\right)\right) ; \%$ BER calculation

BER_B $=(1 / 2)^{*}\left(\operatorname{erfc}\left(\left(\left(\operatorname{SNR} \_B\right) \cdot{ }^{\wedge} 0.5\right) /(2 * \operatorname{sqrt}(2))\right)\right) ; \%$ BER calculation

for $\mathrm{i}=1: 5$

A_new(i) $=$ One(i);

B_new(i) $=$ One $(i, 2)$;

end;

semilogy( A_new, BER_A, '-*', B_new, BER_B, '-o')

grid on

legend ('One leg', 'Two leg')

xlabel('Electrical input voltage[V], both input are varying with equal')...

('rate, only quantum noise considered.');

ylabel('BER');

title('Plot of comparison for one leg and two leg input models');

legend ( 'One leg', 'Two leg')

xlabel('Electrical input voltage[V], both input are varying with equal rate');

ylabel('BER');

title('Plot of comparison for one leg and two leg input models'); 


\section{Appendix C}

\section{Bessel Function of the First Kind}

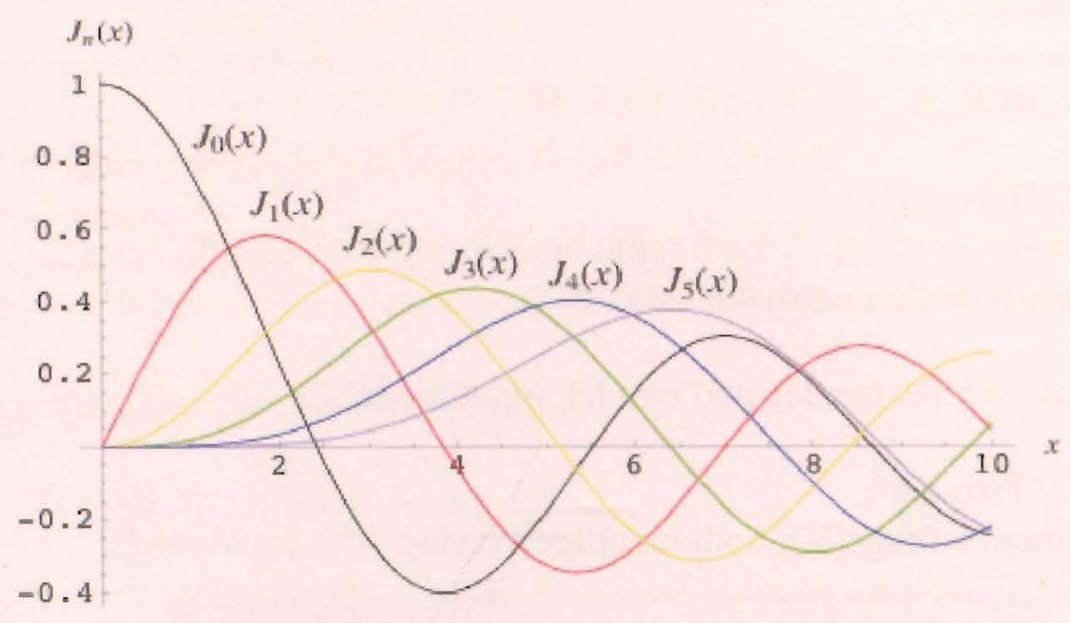

The Bessel functions of the first kind $J_{n}(x)$ are defined as the solutions to the Bessel differential equation

$$
x^{2} \frac{d^{2} y}{d x^{2}}+x \frac{d y}{d x}+\left(x^{2}-n^{2}\right) y=0
$$

which are nonsingular at the origin. They are sometimes also called cylinder functions or cylindrical harmonics. The above plot shows $J_{n}(x)$ for $n=0,1,2, \ldots, 5$. The notation ${ }^{J_{2}}$ was first used by Hansen (1843) and subsequently by Schlömilch (1857) to denote what is now written ${ }_{n}{ }^{(2 z)}$ (Watson 1966 , p. 14). However, Hansen's definition of the function itself in terms of the generating function

$$
e^{z(t-1, t i n) / 2}=\sum_{n=-\infty}^{\infty} f^{n} J_{n}(z)
$$

is the same as the modern one (Watson 1966, p. 14). Bessel used the notation $I_{k}^{f_{k}}$ to denote what is now called the Bessel function of the first kind (Cajori 1993, vol. 2, p. 279).

The Bessel function ${ }^{J_{n}(z)}$ can also be defined by the contour integral 


$$
J_{n}(\varepsilon)=\frac{1}{2 \pi i} \oint e^{(i ; 2)(s-1 ; n)} t^{-n-1} d t
$$

where the contour encloses the origin and is traversed in a counterclockwise direction (Arfken 1985, p. 416).

The Bessel function of the first kind is implemented in Mathematica as BesselJ $[n u, z]$.

To solve the differential equation, apply Frobenius method using a series solution of the form

$$
y=x^{k} \sum_{n=0}^{\infty} a_{n} \cdot x^{n}=\sum_{n=0}^{\infty} a_{n} \cdot x^{n+k}
$$

Plugging into ( 1 ) yields

$$
\begin{aligned}
& x^{2} \sum_{n=0}^{\infty}(k+n)(k+n-1) a_{n} x^{k+n-2}+x \sum_{n=0}^{\infty}(k+n) a_{n} x^{k+n-1}+x^{2} \sum_{n=0}^{\infty} a_{n} x^{k+n}-m^{2} \sum_{n=0}^{\infty} a_{n} x^{n+k}=0 \\
& \sum_{n=0}^{\infty}(k+n)(k+n-1) a_{n} x^{k+n}+\sum_{n=0}^{\infty}(k+n) a_{n} x^{k+n} \\
& +\sum_{n=2}^{\infty} a_{n-2} x^{k+n}-m^{2} \sum_{n=0}^{\infty} a_{n} x^{n+k}=0 .
\end{aligned}
$$

The indicial equation, obtained by setting $n=0$, is

$$
a_{0}\left[k(k-1)+k-m^{2}\right]=a_{0}\left(k^{2}-m^{2}\right)=0 .
$$

Since $a_{0}$ is defined as the first nonzero term, $k^{2}-m^{2}=0$, so $k= \pm m$. Now, if $k=m$,

$$
\begin{aligned}
& \sum_{n=0}^{\infty}\left[(m+n)(m+n-1)+(m+n)-m^{2}\right] a_{n} x^{m+n}+\sum_{n=2}^{\infty} a_{n-2} x^{m+n}=0 \\
& \sum_{n=0}^{\infty}\left[(m+n)^{2}-m^{2}\right] a_{n} x^{m+n}+\sum_{n=2}^{\infty} a_{n-2} x^{m+n}=0 \\
& \sum_{n=0}^{\infty} n(2 m+n) a_{n} x^{m+n}+\sum_{n=2}^{\infty} a_{n-2} x^{m+n}=0 \\
& a_{1}(2 m+1) x^{m+1}+\sum_{n=2}^{\infty}\left[a_{n} n(2 m+n)+a_{n-2}\right] x^{m+n}=0 .
\end{aligned}
$$

First, look at the special case $m=-1 / 2$, then (11) becomes 


$$
\sum_{n=2}^{\infty}\left[a_{n} n(n-1)+a_{n-2}\right] x^{m+n}=0
$$

so

$$
a_{n}=-\frac{1}{n(n-1)} a_{n-2}
$$

Now let $n \equiv 2 l$, where $l=1,2, \ldots$

$$
\begin{aligned}
a_{21} & -\frac{1}{2 l(2 l-1)} a_{2 l-2} \\
& =\quad \frac{(-1)^{\prime}}{[2 !(2 l-1)][2(l-1)(2 l-3)] \cdots[2 \cdot 1 \cdot 1]} a_{0} \\
& =\frac{(-1)^{\dagger}}{2^{l} l !(2 l-1) ! !} a_{0} .
\end{aligned}
$$

which, using the identity $2^{l} l !(2 l-1) ! !=(2 l) !$, gives

$$
a_{2}=\frac{(-1)^{\prime}}{(2 n) !} a_{0}
$$

Similarly, letting $n \equiv 2 l+1$,

$$
a_{2 t+1}=-\frac{1}{(2 t+1)(2 t)} a_{2 t-1}=\frac{(-1)^{\prime}}{[2 t(2 t+1)][2(t-1)(2 t-1)] \cdots[2 \cdot 1 \cdot 3 \mid[1]} a_{1+}
$$

which, using the identity $2^{l} l !(2 l+1) ! !=(2 l+1) !$, gives

$$
a_{2 l+1}=\frac{(-1)^{\prime}}{2^{\prime} l !(2 l+1) ! !} a_{1}=\frac{(-1)^{\prime}}{(2 l+1) !} a_{1} .
$$

Plugging back into (4) with $k=m=-1 / 2$ gives

$$
\begin{aligned}
& y \quad=\quad x^{-1 / 2} \sum_{n=0}^{w} a_{n} x^{n} \\
& =\quad x^{-1 / 2}\left[\sum_{n=1,3, \ldots}^{\infty} a_{n} x^{n}+\sum_{n=024 \ldots}^{\infty} a_{n} x^{n}\right] \\
& =\quad x^{-1 / 2}\left[\sum_{i=0}^{\infty} a_{2 f} x^{2 l}+\sum_{l=0}^{\infty} a_{2 f+1} x^{2 t+1}\right]
\end{aligned}
$$




$$
\begin{array}{ll}
= & x^{-1 / 2}\left[a_{0} \sum_{i=0}^{\infty} \frac{(-1)^{l}}{(2 l) !} x^{2 t}+a_{1} \sum_{l=0}^{\infty} \frac{(-1)^{t}}{(2 l+1) !} x^{2 l+1}\right] \\
= & x^{-1 / 2}\left(a_{0} \cos x+a_{1} \sin x\right) .
\end{array}
$$

The Bessel functions of order $\pm 1 / 2$ are therefore defined as

$$
\begin{aligned}
J_{-1 / 2}(x) & \equiv \sqrt{\frac{2}{\pi x}} \cos x \\
J_{1 / 2}(x) & \equiv \sqrt{\frac{2}{\pi x}} \sin x
\end{aligned}
$$

so the general solution for $m= \pm 1 / 2$ is

$$
y=a_{0}^{\prime} J_{-1 / 2}(x)+a_{1}^{\prime} J_{1 / 2}(x) .
$$

Now, consider a general $m \neq-1 / 2$. Equation (20) requires

$$
\begin{aligned}
& a_{1}(2 m+1)=0 \\
& {\left[a_{n} n(2 m+n)+a_{n-2}\right] x^{m+n}=0}
\end{aligned}
$$

for $n=2,3, \ldots$, so

$$
\begin{array}{lll}
a_{1} & = & 0 \\
a_{n} & = & -\frac{1}{n(2 m+n)} a_{n-2}
\end{array}
$$

for $n=2,3, \ldots$. Let $n \equiv 2 l+1$, where $l=1,2, \ldots$, then

$$
\begin{aligned}
a_{2 l+1} & =\quad-\frac{1}{(2 l+1)[2(m+n)+1]} a_{2 l-1} \\
& =\quad \ldots=f(n, m) a_{1}=0,
\end{aligned}
$$

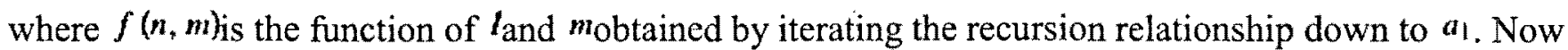
let $n \equiv 2 l$, where $l=1,2, \ldots$, so

$$
\begin{aligned}
a_{2 l} & = & -\frac{1}{2 l(2 m+2 h)} a_{2 \ell-2} \\
& = & -\frac{1}{4 l(m+h)} a_{2 l-2}
\end{aligned}
$$




$$
=\quad \frac{(-1)^{4}}{[4 l(m+1)][4(1-1)(m+1-1)] \cdots[4 \cdot(m+1)]} a_{0} .
$$

Plugging back into (11),

$$
\begin{aligned}
& y \quad=\quad \sum_{n=0}^{\infty} a_{n} x^{n+m}=\sum_{n=1,3, \ldots}^{\infty} a_{n} x^{n+m+n}+\sum_{n=02, \ldots \ldots}^{\infty} a_{n} x^{n+m} \\
& =\quad \sum_{l=0}^{\infty} a_{2 l+1} x^{2 l+m+1}+\sum_{l=0}^{\infty} a_{2 l} x^{2 l+m} \\
& =\quad a_{0} \sum_{l=0}^{\infty} \frac{(-1)^{l}}{[4 l(m+l)][4(l-1)(m+l-1)] \cdots[4(m+1)]} x^{2} \\
& =\quad a_{0} \sum_{l=0}^{\infty} \frac{\left[(-1)^{\prime} m(m-1) \cdots 1\right] \cdot x^{2 l+m}}{[4 l(m+l)][4(l-1)(m+1-1)] \cdots[4(m+1) m} . \\
& =\quad a_{0} \sum_{l=0}^{m} \frac{(-1)^{l} m !}{2^{2 !} l !(m+n) !} x^{2 l+m} \text {. }
\end{aligned}
$$

Now define

$$
J_{m}(r) \equiv \sum_{i=0}^{m} \frac{(-1)^{\prime}}{2^{2 l+m} l !(m+l) !} x^{2 l+m}
$$

where the factorials can be generalized to gamma functions for nonintegral $m$. The above equation then becomes

$$
y=a_{0} 2^{m} m ! J_{m}(r)=a_{0}^{\prime} J_{m}(x)
$$

Returning to equation (11) and examining the case $k=-m$,

$$
a_{1}(1-2 m)+\sum_{n=2}^{\infty}\left[a_{n} n(n-2 m)+a_{n-2}\right] x^{n-m}=0 .
$$

However, the sign of $m$ is arbitrary, so the solutions must be the same for $+m$ and $-m$. We are therefore free to replace $-m_{\text {with }}-|m|$, so

$$
a_{1}(1+2|m|)+\sum_{n=2}^{\infty}\left[a_{n} n(n+2|m|)+a_{n-2}\right] x^{|m|+n}=0 .
$$


and we obtain the same solutions as before, but with mreplaced by $|m|$.

$$
J_{m}(x)= \begin{cases}\sum_{l=0}^{\infty} \frac{(-1)^{\prime}}{2^{2 l+k n \mid} l !(|m|+n) !} x^{2 l+h r \mid} & \text { for }|m| \neq \frac{1}{2} \\ \sqrt{\frac{2}{\pi x} \cos x} & \text { for } m=-\frac{1}{2} \\ \sqrt{\frac{2}{\pi x}} \sin x & \text { for } m=\frac{1}{2}\end{cases}
$$

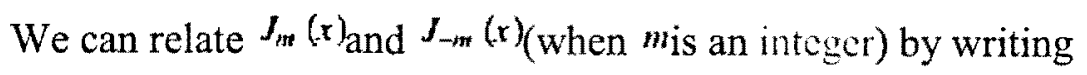

$$
J_{-m}(x)=\sum_{t=0}^{\infty} \frac{(-1)^{t}}{2^{2 t-m} l !(l-m) !} \cdot x^{2 l-m}
$$

Now let $l \equiv l^{\prime}+m$. Then

$$
\begin{aligned}
& J_{-m} t \quad \sum_{r^{\prime}+m=0}^{\infty} \frac{(-1)^{y^{\prime}+m}}{2^{2 l^{r}+m}\left(l^{r}+m\right) ! ! !} x^{2 r^{r}+m} \\
& =\quad \sum_{r^{\prime}=-m}^{-1} \frac{(-1)^{\prime \prime+m}}{2^{2 r^{\prime}+m} l^{\prime} !\left(l^{\prime}+m\right) !} x^{2 r^{\prime}+m}+\sum_{r^{\prime}=0}^{\infty} \frac{(-1)^{r^{\prime}+m}}{2^{2 r^{\prime}+m} l^{\prime} !\left(l^{\prime}+\right.}
\end{aligned}
$$

But $l^{\prime} !=\infty$ for $t^{\prime}=-m, \ldots,-1$, so the denominator is infinite and the terms on the left are zero. We therefore have

$$
\begin{aligned}
J_{-m}(x) \quad & \sum_{l=0}^{\infty} \frac{(-1)^{j+m}}{2^{2 l+m} ! !(1+m) !} x^{21+m} \\
& =\quad(-1)^{n} J_{m}(x) .
\end{aligned}
$$

Note that the Bessel differential equation is second-order, so there must be two linearly independent solutions. We have found both only for $|m|=1 / 2$. For a general nonintegral order, the independent solutions are $J_{m \text { and }} J_{-m}$. When $m_{\text {is }}$ an integer, the general (real) solution is of the form

$$
Z_{m} \equiv C_{1} J_{m}(x)+C_{2} Y_{m}(x)
$$

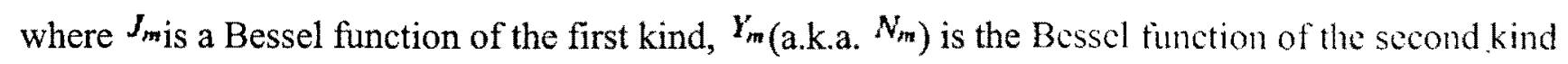
(a.k.a. Neumann function or Weber function), and $C_{1}$ and $C_{2}$ are constants. Complex solutions are given 
by the Hankel functions (a.k.a. Bessel functions of the third kind).

The Bessel functions are orthogonal in $[0, a]$ according to

$$
\int_{b}^{k} J_{v}\left(\alpha_{v, m} \frac{\rho}{a}\right) J_{v}\left(\alpha_{v \pi} \frac{\rho}{a}\right) \rho d \rho=\frac{1}{2} a^{2}\left[J_{v+1}\left(\alpha_{v m}\right)\right]^{2} \delta_{m n}
$$

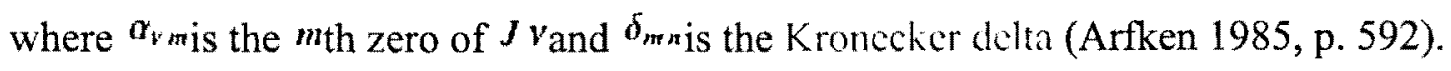

Except when $2 m$ is a negative integer,

$$
J_{m}(z)=\frac{z^{-1 / 2}}{2^{2 m+1 / 2} i^{m+1 / 2} \Gamma(m+1)} M_{0 m m}(2 i z)
$$

where $\Gamma(r)_{\text {is }}$ the gamma function and $M_{O r}$ is a Whittaker function.

In terms of a confluent hypergeometric function of the first kind, the Bessel function is written

$$
J_{v}(z)=\frac{\left(\frac{1}{2} z\right)^{v}}{\Gamma(v+1)} \circ F_{1}\left(v+1 ;-\frac{1}{4} z^{2}\right)
$$

A derivative identity for expressing higher order Bessel functions in terms of $J_{0}(z)_{\text {is }}$

$$
J_{n}(\varepsilon)=i^{n} T_{n}\left(i \frac{d}{d z}\right) J_{0}(\xi)
$$

where $T_{n}(\varepsilon)$ is a Chebyshev polynomial of the first kind. Asymptotic forms for the Bessel functions are

$$
J_{m}(\varepsilon) \approx \frac{1}{\Gamma(m+1)}\left(\frac{z}{2}\right)^{m}
$$

for $z \ll 1$ and

$$
J_{m}(z) \approx \sqrt{\frac{2}{\pi z}} \cos \left(z-\frac{m \pi}{2}-\frac{\pi}{4}\right)
$$

for $z \gg\left|m^{2}-1 / 4\right|$ (correcting the condition of Abramowitz and Stegun 1972, p. 364).

A derivative identity is 


$$
\frac{d}{d x}\left[x^{m} J_{n}(x)\right]=x^{m} J_{m-1}(x)
$$

An integral identity is

$$
\int_{0}^{\prime \prime} u^{\prime} J_{0}\left(u^{\prime}\right) d u^{\prime}=u J_{1}(u)
$$

Some sum identities are

$$
\sum_{k=-\infty}^{\infty} J_{k}(r)=1
$$

(which follows from the generating function (4) with $t=1$ ),

$$
l=\left[J_{0}(r)\right]^{2}+2 \sum_{k=1}^{\infty}\left[J_{k}(r)\right]^{2}
$$

(Abramowitz and Stegun 1972, p. 363),

$$
1=J_{0}(x)+2 \sum_{k=1}^{\infty} J_{2 k}(r)
$$

(Abramowitz and Stegun 1972, p. 361),

$$
0=\sum_{k=0}^{2 n}(-1)^{k} J_{k}(z) J_{2 n-k}(z)+2 \sum_{k=1}^{m} J_{k}(z) J_{2 n+k}(\sigma)
$$

for $n \geq 1$ (Abramowitz and Stegun 1972, p. 361),

$$
J_{n}(2 z)=\sum_{k=0}^{n} J_{k}(z) J_{n-k}(z)+2 \sum_{k=1}^{\infty}(-1)^{k} J_{k}(z) J_{n+k}(z)
$$

(Abramowitz and Stegun 1972, p. 361), and the Jacobi-Anger expansion

$$
e^{i z \cos \theta}=\sum_{n=-\infty}^{\infty} i^{n} J_{n}(z) e^{i n \theta}
$$

which can also be written 


$$
e^{i z \cos \theta}=J_{0}(z)+2 \sum_{n=1}^{n} i^{n} J_{n}(z) \cos (n \theta)
$$

The Bessel function addition theorem states

$$
J_{n}(v+z)=\sum_{m=-\infty}^{\infty} J_{m}(y) J_{n-m}(z)
$$

Various integrals can be expressed in terms of Bessel functions

$$
J_{n}(\bar{m})=\frac{1}{\pi} \int^{\pi} \cos (z \sin \theta-n \theta) d \theta
$$

which is Bessel's first integral,

$$
\begin{array}{ll}
J_{n}(z) & =\frac{i^{-n}}{\pi} \int^{\pi} e^{j z \cos t \theta} \cos (n \theta) d \theta \\
J_{n}(\xi) & =\frac{1}{2 \pi i^{n}} \int^{2 n} e^{2 \pi \cos \theta} e^{i n \phi} d \phi
\end{array}
$$

for $n=1,2, \ldots$,

$$
J_{n}(z)=\frac{2}{\pi} \frac{z^{n}}{(2 n-1) ! !} \int_{0}^{\pi / 2} \sin ^{2 n} u \cos (z \cos u) d u
$$

for $n=1,2, \ldots$,

$$
J_{n}(x)=\frac{1}{2 \pi i} \int_{\gamma} e^{(x / 2)(z-1 / i)} z^{-n-1} d z
$$

for $n>-1 / 2$. The Bessel functions are normalized so that

$$
\int_{0}^{\infty} J_{n}(x) d x=1
$$

